\title{
Reductions in representation theory of Lie algebras of vector fields
}

\author{
André Eduardo Zaidan
}

A thesis SubmitTed to the FaCulty of GRAduAte and Postdoctoral Affairs Carleton University And Instituto de Matemática e Estatística dA Universidade DE SÃo PAUlO IN PARTIAL FULFILLMENT OF THE REQUIREMENTS FOR THE DEGREE OF Doctor of Philosophy in Mathematics

\author{
Program: Mathematics \\ Supervisors: Yuly Billig, Ph.D. \\ Vyacheslav Futorny, Ph.D.
}

During the development of the present work the author received financial assistance of CAPES process: 88881.133701/2016-01

São Paulo, April 2020 


\section{Reductions in representation theory of Lie algebras of vector fields}

This is the original version of the thesis elaborated by the candidate André Eduardo Zaidan, as it was submitted to the examination committee. 


\section{Abstract}

\section{Zaidan, A. E. Reductions in representation theory of Lie algebras of vector}

fields. 2020. 61 p. Thesis (Ph.D.) - Instituto de Matemática e Estatística, Universidade de São Paulo and Carleton University, 2020.

We study representations of Lie algebras that do not have a Cartan subalgebra. The study of such representations required new techniques, one that we applied was to restrict the action of other algebraic structures that contain the Lie algebra.

Our Lie algebras came from the vector fields on arbitrary varieties. We studied representations that admit the actions of the Lie algebra of vector field and the algebra of functions on the variety in a compatible way. More specifically, we studied two such classes of modules: gauge modules and Rudakov modules.

We proved that gauge modules and Rudakov modules corresponding to simple $\mathfrak{g l}_{N^{-}}$ modules remain irreducible as modules over the Lie algebra of vector fields unless they appear in the de Rham complex. We also studied the irreducibility of tensor products of Rudakov modules.

Lastly, we present a complete description of tensor modules belonging to the de Rham complex as $\mathfrak{g l}_{3}$-modules. We also realize these modules using GT-tableaux.

Keywords: gauge modules, Lie algebra of vector fields, Rudakov modules. 


\section{Resumo}

Zaidan, A. E. Reduções na teoria de representações das álgebras de Lie de campos vetoriais. 2020. 61 p. Tese (Doutorado) - Instítuto de Matemática e Estatística, Universidade de São Paulo e Universidade de Carleton, São Paulo, 2020.

Nós estudamos representações para álgebras de Lie que não possuem uma subalgebra de Cartan. O estudo de tais representções requer novas técnicas, a que nós aplicamos consiste em restringir a ação de outras estruturas algébricas que contenham a álgebra de Lie.

Nossas álgebras de Lie são obtidas a partir de campos vetoriais de variedades arbitrárias. Nós estudamos representações que admitem a ação da álgebra de Lie de campos vetoriais e a ação da álgebra de funções na variedade de uma maneira compatível. Mais especificamente, estudamos duas classes de tais módulos: módulos de gauge e módulos de Rudakov.

Nós provamos que módulos de gauge e módulos de Rudakov com o correspondente $\mathfrak{g l}_{N}$-módulo simples continuam irredutíveis como módulos sobre a álgebra de Lie de campos vetoriais, a menos que o módulos apareça no complexo de de Rham. Nós também estudamos irreducibilidade do produto tensorial de módulos de Rudakov.

Por fim, nós apresentamos uma descrição de módulos tensoriais que pertencem ao complexo de de Rham como $\mathfrak{g l}_{3}$-módulos. Nós também realizamos tais módulos via tabelas GT.

Palavras chave: álgebra de Lie de campos vetoriais, módulos de gauge, módulos de Rudakov. 


\section{Contents}

1 Introduction 1

1.1 Historical background . . . . . . . . . . . . . . . . . . 1

1.2 Motivation . . . . . . . . . . . . . . . . . . . 3

1.3 Main results . . . . . . . . . . . . . . . . . . . . . . 4

2 Notation and Definitions $\quad 7$

2.1 Lie algebras . . . . . . . . . . . . . . . . . . . . . 7

2.2 Representations . . . . . . . . . . . . . . . . . . . . 13

2.2.1 Representations of associative algebras . . . . . . . . . . . . . 14

2.2.2 Universal enveloping algebra . . . . . . . . . . . . . . . 15

2.2 .3 Representations of Lie algebras . . . . . . . . . . . . . . 17

2.3 Algebraic varieties . . . . . . . . . . . . . . . . . . . . . 22

$2.4 \mathcal{A} \mathcal{V}$ - modules . . . . . . . . . . . . . . . . . 25

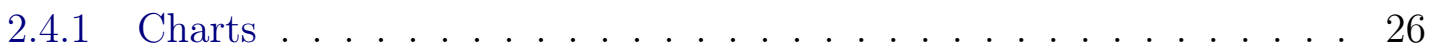

3 Gauge Modules $\quad 31$

3.1 Tensor modules . . . . . . . . . . . . . . . . . . . . . 31

3.2 Gauge fields . . . . . . . . . . . . . . . . . . . . . . . . . 32

3.3 De Rham complex . . . . . . . . . . . . . . . . . . . . . . . 37

3.4 Gauge modules on $\mathbb{S}^{1}$ and irreducible modules for $\mathfrak{s l}_{2} \ldots \ldots$. . . . . . . 40

4 Rudakov Modules $\quad 43$

4.1 Rudakov modules for arbitrary varieties . . . . . . . . . . . . . . . . . . 44

4.2 Tensor Product of Rudakov Modules . . . . . . . . . . . . . . . . . . . 48

5 Realizations of de Rham Complex $\quad 51$

$5.1 \mathfrak{g l}_{N+1}$-modules from $\mathcal{V}_{\mathbb{T}^{N} \text {-module } \ldots \ldots \ldots \ldots \ldots} \ldots \ldots \ldots$

5.2 Gelfand-Tsetlin-modules for $\mathfrak{g l}_{N} \ldots \ldots \ldots \ldots$

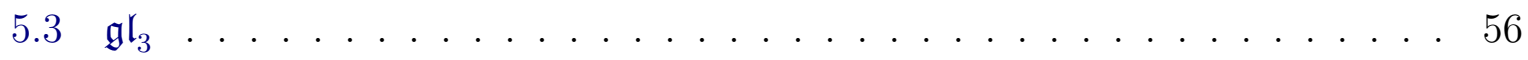

$\begin{array}{ll}\text { Bibliography } & 59\end{array}$ 
vi CONTENTS

Index

61 


\section{Chapter 1}

\section{Introduction}

This thesis is organized into five chapters. The present one contains a historical background and the motivation for our work. It also presents our main results. The second chapter contains the basic material about Lie algebras, algebraic geometry and representation theory that is necessary to the reader in order to follow our results. It also contains some highlights of previous results that will be needed in the text. Readers who are familiar with these subjects can go directly to the last section of that chapter, where we present some important results concerning the Lie algebra of vector fields.

The third and fourth chapters are devoted to gauge modules and Rudakov modules, respectively. These are the two classes of modules that we studied. These modules have been studied before as modules over both the Lie algebra of vector fields and the algebra of functions on a variety.

In the fifth chapter, we describe a family of $\mathfrak{g l}_{3}$-modules obtained from the de Rham complex of tensor modules for the algebra of vector field on an 2-dimensional torus.

\section{$1.1 \quad$ Historical background}

One of the main goals in science is to find patterns in nature; symmetries are a great source of them. The study of symmetries has proven to be very fruitful, an example is an important theorem due to Emmy Noether which, roughly speaking, states that every symmetry in a physical system will correspond to a conservation law. For example, the translational symmetry of the space corresponds to the conservation of the linear momentum.

In mathematics, the formalization of symmetries was implemented using the notion of a group. Since symmetries arise from almost everywhere, it is not clear where the abstract group theory, as we know it, exactly started. Nevertheless, one of the major accomplishments at the start of the development of the theory was the study of the symmetries of the roots of algebraic equations done by Lagrange [22]. Years later, Galois built a bridge between field theory and group theory. One of the most notable achievements was a simpler proof of the non-existence of a solution by radicals of polynomial equations of degree five or more.

The theory developed by Galois was admired by many, including the Norwegian mathematician Sophus Lie. Aiming to create a version of Galois Theory for differential equations, he created groups using an analytic point of view, which he called groups of transformations and nowadays are called Lie groups. Examples of Lie groups are the set of non-singular $n$ by $n$ matrices denoted $\mathrm{GL}_{n}$ and its subgroup of matrices with determinant 
1 , denoted $\mathrm{SL}_{n}$.

The approach used by Lie was to look for transformations in the variables of a differential equation in order to decide if it is solvable in quadratures, i.e., has a solution that can be expressed by integrals.

Not many years after the formulation given by Sophus Lie, Wilhelm Killing [21] and Élie Cartan [10] classified all simple Lie groups, which can be seen as the atoms of the theory. The classification was done by looking for an algebraic structure that codified the local structure of a Lie group, this structure is called Lie algebra. The Lie algebra of a Lie group is the tangent space at the identity. From a Lie algebra, one can recover the Lie group through an exponential map. For the fields $\mathbb{R}$ and $\mathbb{C}$ the image of exponential map can be seen as the curve that that is tangent to the group at the identity. The existence of such a map is what makes the study of Lie groups through Lie algebras possible. For example, for connected and simply connected Lie groups, their Lie algebras are isomorphic if and only if the groups are isomorphic.

To classify all simple finite-dimensional Lie algebras, Killing managed to show that the whole algebra may be codified in an even simpler structure, namely, its root system.

The idea is to find a suitable subalgebra, which elements act on the whole algebra in a diagonalizable way, such subalgebra is called a Cartan subalgebra. A root system consists of a set of eigenvectors for a basis of such subalgebra. The root system is uniquely determined for each simple Lie algebra and, also, each root system is related to a unique simple finite-dimensional complex Lie algebra up to isomorphism.

It is not an overstatement to say that this technique shaped the development of the theory, for example, the study of infinite-dimensional Lie algebras is very entangled with root systems. The four classes of Lie algebras of Cartan type, the general series $\mathbf{W}_{n}$, the special series $\mathbf{S}_{n}$, the Hamiltonian series $\mathbf{H}_{n}$ and the contact series $\mathbf{K}_{n}$, were the first examples of simple infinite-dimensional Lie algebras, they were known since the time of Sophus Lie. However, there was no significant development after the work of Cartan on infinite-dimensional Lie algebras until the decade of 1960. Many types of Lie algebras were studied, vector fields on manifolds, smooth mappings of a manifold into a finite dimensional Lie algebra, and operators on a Hilbert space are some examples. But a truly profound insight into infinite-dimensional Lie algebras was gained with the independent works of Victor Kac [20] and Robert Moody [27]. They generalized simple finite-dimensional Lie algebras relaxing the restrictions on root systems.

Another great example that was influenced by roots is representation theory. In algebra, representation theory is a natural way to see how some algebraic structure acts on a vector space. The main tool in representation theory for Lie algebras is a generalization of roots called weights. Essentially, the idea is to look for modules where the action of the Cartan subalgebra is diagonalizable. A module with such a property is called a weight module. Such modules have been extensively studied. For instance, for complex finite-dimensional Lie algebras, every finite-dimensional module is a weight module.

The classification of all irreducible representations was only made for the Lie algebra of 2 by 2 matrices with trace zero over a field of characteristic zero, in a work by Block [9]. For other Lie algebras, even the classification of weight modules is not complete. In order to make a classification feasible one can impose some finiteness condition. For example, classification of modules with finite or uniformly bounded dimensions of weight spaces, which are the generalized eigenspaces for the Cartan subalgebra. For certain algebras, the classification of modules with weight spaces of finite or bounded dimensions has been completed. A theory without roots and, consequently, without weights is still largely 
undeveloped.

Lie algebras have been widely used to solve many questions about Lie groups. But its theory has proliferated into many other areas. Algebraic geometry, combinatorics, computer vision, conformal field theory, harmonic analysis, particle physics, quantum groups, quantum physics, string theory, are some examples. Another, particularly interesting, area that the theory of Lie algebras influenced is gauge theory.

Gauge theory deals with the fact that we can make some changes on fields without changing the physics beneath it. For example, adding a constant field to the gravitational or electric potential fields does not change the physics. For the magnetic potential field, we can add a field with curl zero and the physics would not change. These types of changes are symmetries in the sense of Emmy Noether's theorem and for each system form a Lie algebra (see [1] for more details).

This shows us how important is the study of Lie algebras and their representations. The area of Lie algebras without a Cartan subalgebra is, however, fairly undeveloped and in this work we show some techniques of how to obtain representations of such algebras.

\subsection{Motivation}

Witt algebras $\mathbf{W}_{n}$ were first defined as the polynomial vector fields on the affine space, which can be seen as the Lie algebras of derivations of polynomials in $n$ variables. The same name is also used for the Lie algebras of polynomial vector fields on $n$-dimensional torus, which can be seen as the derivations of Laurent polynomials. So one way to produce new Lie algebras is to look at other varieties.

Fix an affine algebraic variety $X$. One source of Lie algebras is the set of maps from $X$ to a Lie algebra $\mathfrak{g}$. One can even go a step further, let $G$ be a group acting on both the Lie algebra and the affine variety. Then the set of equivariant maps under the action of $G$ is a Lie algebra. This is a very large class of algebras and [24] gives a survey on this subject.

Another source of Lie algebras is the vector fields on $X$. Our previous examples, the vector fields on the affine space and the $n$-dimensional torus, are simple Lie algebras. In fact, David Jordan proved simplicity of all Lie algebras of vector fields on smooth irreducible affine varieties [18] (see also [19]).

For some varieties, both types of Lie algebras have been deeply studied and yield a successful representation theory. For example, for $X=\mathbb{S}^{1}$ the first case became the untwisted loop algebra $\mathbb{K}\left[t, t^{-1}\right] \otimes \mathfrak{g}$. For the second, we obtain the famous Virasoro Lie algebra, whose classification of irreducible modules with finite-dimensional weight spaces was established by Mathieu [25]. This result was generalized by Billig and Futorny [5] for an $N$-dimensional torus.

An important part of these classifications was done by looking for modules that admit the action of both the polynomial algebra $\mathcal{A}$ on $X$ and the algebra of vector field $\mathcal{V}$ on $X$ in a compatible way. Such modules are called $\mathcal{A} \mathcal{V}$-modules.

For an arbitrary variety, these algebras present many challenges. For instance, even to describe their elements can be somewhat tricky. The existence of Cartan subalgebra is not guaranteed, so the main tools of representation theory, roots and weights, are not applicable. Finiteness conditions were not known at the time, so even to conjecture how to generalize the previous results was not clear.

In [6], Billig and Futorny described a new way to present the algebra of vector fields. Using this technique together with the Hilbert Nullstellensatz, Billig and Nilsson [8] proved 
the simplicity of tensor modules for the algebra of vector fields on a sphere. For an arbitrary variety Billig, Futorny and Nilsson [7] studied representations in the category of $\mathcal{A} \mathcal{V}$-modules. Specifically, two classes of representation were studied: Rudakov modules and gauge modules.

The basic ingredient to these two classes of modules is $\mathfrak{g l}_{N}$-modules. Gauge modules are generalizations of tensor modules, which are constructed from modules of vector fields and differential 1-forms by means of taking iterated tensor products parametrized by finite-dimensional $\mathfrak{g l}_{N}$-modules. Rao [28] proved that tensor modules that were generated from irreducible $\mathfrak{g l}_{N}$-modules were irreducible $\mathbf{W}_{N}$-modules for the $N$-dimensional torus, unless the $\mathfrak{g l}_{N}$ module was a power of the natural module. The difference between tensor modules with gauge modules is the introduction of gauge fields.

In [29], Rudakov studied modules for the four Cartan type Lie algebras. Prior to his work, representations of infinite-dimensional Lie algebras were known for Virasoro algebra and finite-dimensional representations of Lie algebras of Cartan type were studied in positive characteristic. His idea consisted in reducing the problem of classifying modules for these infinite-dimensional Lie algebras to the problem of classifying modules for finitedimensional Lie algebras. For the algebra $\mathbf{W}_{N}$ he constructed a class of induced modules, the top of such modules are the $\mathfrak{g l}_{N}$-modules. He has shown that these modules are irreducible if the $\mathfrak{g l}_{N}$-module is irreducible and is not the dual of some exterior power of the natural module. These modules were generalized in [7] for arbitrary varieties and are called Rudakov modules.

The main result of [7] and our starting point is that gauge modules and Rudakov modules that come from irreducible $\mathfrak{g l}_{N}$-modules are irreducible as $\mathcal{A} \mathcal{V}$-modules.

\subsection{Main results}

From the main result of [7], gauge and Rudakov modules are irreducible as $\mathcal{A} \mathcal{V}$-modules as long as the $\mathfrak{g l}_{n}$-module is irreducible. One attempt to obtain irreducible $\mathcal{V}$-modules is to restrict the action. When restricting an action it is not guaranteed that an irreducible module will remain irreducible. Surprisingly, in our case, all, except finitely many, of the $\mathcal{A} \mathcal{V}$-modules remain irreducible when viewed as $\mathcal{V}$-modules.

For the gauge modules, the proof is divided into two parts. The first part consists of showing that we can reconstruct the $\mathcal{A}$ action from the $\mathcal{V}$ action, except for a finite number of $\mathfrak{g l}_{N}$-modules. When reconstructing the $\mathcal{A}$-action, we get restrictions on the central character of the $\mathfrak{g l}_{N}$-module. This was interesting because it was independent of the variety $X$ or the gauge fields. Also, we were not able to reconstruct the $\mathcal{A}$-action for only a finite number of central characters, and by the Harish-Chandra isomorphism we know that a finite-dimensional irreducible $\mathfrak{g l}_{N}$-module is uniquely determined by its central character. So, we had at most a finite number of modules for which we could not claim irreducibility by this technique. For the second part, we have shown that gauge modules for which we cannot claim irreducibility are, in fact, reducible and the corresponding $\mathfrak{g l}_{N^{-}}$ modules are one of the exterior powers of the natural module. This is summarized as our main theorem on gauge modules:

Theorem. If $U$ is a simple finite-dimensional $\mathfrak{g l}_{N}$-module that is not an exterior power of the natural module, then any gauge module corresponding to $U$ is simple as a $\mathcal{V}$-module.

For the Rudakov modules, the idea was to make the necessary changes to Rudakov's proof for the affine space, generalizing it to an arbitrary variety. Rudakov modules have 
a filtration. The first part of the proof consists of showing that the first component of the filtration is the only place where we can find singular vectors, i.e., vectors annihilated by the positive part of the algebra and not belonging to the top. The existence of such vectors is deeply linked to the irreducibility of induced modules. The second part of the proof consists of determining the possible central characters for the $\mathfrak{g l}_{N}$-module in order for the Rudakov module to contain a singular vector. This is done by restricting the possible $\mathfrak{g l}_{N}$ actions using equations of the type $\mathcal{V}_{+} u=0$, where $u$ is a singular vector and $\mathcal{V}_{+}$the positive part of $\mathcal{V}$. We obtain that singular vectors exist if and only if $\mathfrak{g l}_{N}$-module is the dual of an exterior power of the natural module. This is our main theorem about Rudakov modules:

Theorem. If $U$ is a simple finite-dimensional $\mathfrak{g l}_{N}$-module that is not the dual of an exterior power of the natural module, then the Rudakov module $R_{p}(U)$ is irreducible as a $\mathcal{V}$-module.

We also proved that the tensor product of irreducible Rudakov modules at distinct base points is irreducible. The proof consists in creating a version of the Chinese remainder theorem for the Lie algebra of vector fields. This was motivated by a result due to Michael Lau [23]. He proved that every finite-dimensional module for multiloop algebras is the tensor product of evaluation modules. Our result motivates the following conjecture:

Conjecture 1. Let $M$ be an irreducible $\mathcal{A} \mathcal{V}$-module in which every vector generates a finite-dimensional $\mathcal{A}$-submodule. Then $M$ is isomorphic to the tensor product of Rudakov modules.

Finally, we constructed $\mathfrak{g l}_{N+1}$-modules by restricting the action of the Lie algebra of vector fields on a $N$-dimensional torus. The modules that we study are the ones appearing in the de Rham complex, these modules are reducible as modules over the Lie algebra of vector fields, we found conditions for the irreducible components to remain irreducible after restricting the action. For the case $N=2$, we gave a complete description of these modules realizing them using GT-tableaux and using the classification given in [14]. 


\section{Chapter 2}

\section{Notation and Definitions}

This chapter is divided into four parts. The first two present the definition of Lie algebras and their representations, the main reference is [17]. The third part is a brief review of algebraic varieties with the main reference [16]. Finally, the fourth part presents the category of $\mathcal{A} \mathcal{V}$-modules. Our objects of study live in that category. The fourth part also contains a construction (due to [4]) describing elements of vector fields on a variety, in a more tangible way.

\subsection{Lie algebras}

An algebra is a vector space with a bilinear product. One way how algebras appear is as the set of observables in physics. For example, the set of observables in classical mechanics is a Poisson algebra while the ones in quantum mechanics is a Jordan algebra.

Another example of algebra is the set of endomorphisms of a vector space with the product being the composition, it is an associative algebra. Our object of study is an algebra that is neither commutative nor associative, instead, it has two properties that substitute commutativity and associativity:

Definition 2. A Lie algebra $\mathfrak{g}$ over a field $\mathbb{K}$ is a $\mathbb{K}$-vector space together with a bilinear map $[.,]:. \mathfrak{g} \times \mathfrak{g} \rightarrow \mathfrak{g}$, called Lie bracket, with the following properties, for any $x, y, z \in \mathfrak{g}$ :

1. $[x, x]=0$,

2. $[x,[y, z]]+[y,[z, x]]+[z,[x, y]]=0$.

The first property implies anticommutativity $[x, y]=-[y, x]$, and in fact, is equivalent to anticommutativity when the characteristic of the field $\mathbb{K}$ is not 2 . The second property is called the Jacobi identity, it is also good to notice that it can be rewritten as $[x,[y, z]]=$ $[[x, y], z]+[y,[x, z]]$ becoming the Leibniz rule for derivatives.

\section{Examples:}

1. Given any vector space $V$ we can define the Lie bracket identically zero. When this is the case we say that Lie algebra is abelian.

2. The space $\mathbb{R}^{3}$ with the cross product as multiplication is a Lie algebra.

3. Associative algebras are a rich source for Lie algebras. Suppose $A$ is an associative algebra where the product is denoted by juxtaposition. We can define a Lie algebra $A^{(-)}$with a Lie bracket given by the commutator, i. e., $[a, b]=a b-b a$ for $a, b \in A$. 
Given two Lie algebras $\mathfrak{g}$ and $\mathfrak{h}$, a linear transformation $\phi: \mathfrak{g} \rightarrow \mathfrak{h}$ is called a homomorphism if $\phi([g, h])=[\phi(g), \phi(h)]$ for all $g, h \in \mathfrak{g}$. If $A$ is an associative algebra, a linear transformation $\psi: \mathfrak{g} \rightarrow A$ is called a Lie associative morphism if $\psi([g, h])=$ $\psi(g) \psi(h)-\psi(h) \psi(g)$ for all $g, h \in \mathfrak{g}$. As usual, a homomorphism that is a bijection is called isomorphism and if the image is equal to the domain is called endomorphism.

Example: Let $\mathfrak{s o}_{n}(\mathbb{K})$ denote the set of skew-symmetric $n$ by $n$ matrices over $\mathbb{K}$. This is a Lie algebra with the bracket given by the commutator. We claim that $\mathfrak{s o}_{3}$ is isomorphic to $\mathbb{K}^{3}$ with the cross product. Indeed, let $\phi: \mathfrak{s o}_{3} \rightarrow \mathbb{K}^{3}$ be given by

$$
\phi\left(\begin{array}{ccc}
0 & a & c \\
-a & 0 & b \\
-c & -b & 0
\end{array}\right)=(a, b, c)
$$

where $a, b, c \in \mathbb{K}$. It is easy to check that $\phi$ is a bijection, we will show that $\phi$ is a homomorphism. We have, in one hand,

$$
\begin{gathered}
\phi\left[\left(\begin{array}{ccc}
0 & a & c \\
-a & 0 & b \\
-c & -b & 0
\end{array}\right),\left(\begin{array}{ccc}
0 & d & f \\
-d & 0 & e \\
-f & -e & 0
\end{array}\right)\right]=\phi\left(\begin{array}{ccc}
0 & b f-c e & a e-b d \\
-(b f-c e) & 0 & -a f+c d \\
-(a e-b d) & -(-a f+c d) & 0
\end{array}\right) \\
=(b f-c e,-a f+c d, a e-b d) .
\end{gathered}
$$

On the other hand

$$
\left[\phi\left(\begin{array}{ccc}
0 & a & c \\
-a & 0 & b \\
-c & -b & 0
\end{array}\right), \phi\left(\begin{array}{ccc}
0 & d & f \\
-d & 0 & e \\
-f & -e & 0
\end{array}\right)\right]=(a, b, c) \times(d, e, f)=(b f-c e,-a f+c d, a e-b d) .
$$

This shows that $\mathfrak{s o}_{3}(\mathbb{K})$ is isomorphic to $\mathbb{K}^{3}$.

Definition 3. Given a (not necessarily associative or commutative) algebra A, the subset of endomorphisms of A that satisfy the Leibniz rule:

$$
\delta(a b)=\delta(a) b+a \delta(b)
$$

is denoted by $\operatorname{Der}(A)$ and its elements are called derivations of $A$.

The composition of derivations is not necessarily a derivation. Nevertheless, the commutator of two derivations is a derivation:

$$
\begin{aligned}
& {[\delta, \gamma](a b)=\delta(\gamma(a b))-\gamma(\delta(a b)) } \\
= & \delta(\gamma(a)) b+\gamma(a) \delta(b)+\delta(a) \gamma(b)+a \delta(\gamma(b))-\gamma(\delta(a)) b-\delta(a) \gamma(b)-\gamma(a) \delta(b)-a \gamma(\delta(b)) \\
= & {[\delta, \gamma](a) b-a[\delta, \gamma](b) . }
\end{aligned}
$$

This gives us another source of Lie algebras - the set of derivations $\operatorname{Der}(A)$ with the Lie bracket given by the commutators.

Definition 4. An ideal of a Lie algebra $\mathfrak{g}$ is a subspace $\mathfrak{h}$ such that $[\mathfrak{g}, \mathfrak{h}] \subset \mathfrak{h}$. A Lie algebra whose dimension is greater than 1 and whose only ideals are 0 and itself will be called simple. 
One important ideal of every Lie algebra is the center:

Definition 5. Let $\mathfrak{g}$ be a Lie algebra, the center of the Lie algebra is defined as

$$
Z(\mathfrak{g}):=\{g \in \mathfrak{g} \mid[g, h]=0, \text { for all } h \in \mathfrak{g}\} .
$$

It follows immediately from the definition that the center is an ideal of $\mathfrak{g}$.

Example: Let us take the algebra of all $n \times n$ matrices over the field $\mathbb{K}$. This associative algebra is a Lie algebra with the Lie bracket given by the commutator and is denoted as $\mathfrak{g l}_{n}(\mathbb{K})$. Since the trace has the property $\operatorname{Tr}(A B-B A)=0$, for any matrices $A, B$, this algebra has two non-trivial ideals: the center, given by multiples of the identity and the subalgebra of matrices with trace equal to zero which we denote by $\mathfrak{s l}_{n}(\mathbb{K})$. The Lie algebra $\mathfrak{s l}_{n}(\mathbb{K})$ is actually our first example of a simple Lie algebra ${ }^{1}$. When the field is fixed we might simply write $\mathfrak{g l}_{n}$ and $\mathfrak{s l}_{n}$.

Let $\mathfrak{g}$ be a Lie algebra and $g, h \in \mathfrak{g}$. Define the adjoint map ad: $\mathfrak{g} \rightarrow \operatorname{Der}(\mathfrak{g})$ as $(\operatorname{ad}(g))(h)=[g, h]$. Because of the Jacobi identity $\operatorname{ad}(g)$ is, indeed, a derivation. Since, for $\delta$ in $\operatorname{Der}(\mathfrak{g}),[\delta, \operatorname{ad}(g)]=\operatorname{ad}(\delta(g))$, we have that $\operatorname{ad}(\mathfrak{g})$ is an ideal of $\operatorname{Der}(\mathfrak{g})$. For simple finite-dimensional Lie algebras we actually have the equality $\operatorname{ad}(\mathfrak{g})=\operatorname{Der}(\mathfrak{g})$ (See section 5.3 of $[17])$.

Example: Let us take the Lie algebra $\mathfrak{s l}_{2}(\mathbb{K})$, the usual basis for this algebra is given as follows:

$$
e=\left(\begin{array}{ll}
0 & 1 \\
0 & 0
\end{array}\right), \quad h=\left(\begin{array}{cc}
1 & 0 \\
0 & -1
\end{array}\right), \quad f=\left(\begin{array}{ll}
0 & 0 \\
1 & 0
\end{array}\right) .
$$

We have $[e, f]=h,[h, e]=2 e$ and $[h, f]=-2 f$, all the others brackets can be obtained immediately using the properties of a Lie algebra. In the basis $\{e, h, f\}$, we have the following matrices for the adjoint:

$$
\operatorname{ad}(e)=\left(\begin{array}{ccc}
0 & -2 & 0 \\
0 & 0 & 1 \\
0 & 0 & 0
\end{array}\right), \quad \operatorname{ad}(h)=\left(\begin{array}{ccc}
2 & 0 & 0 \\
0 & 0 & 0 \\
0 & 0 & -2
\end{array}\right), \quad \operatorname{ad}(f)=\left(\begin{array}{ccc}
0 & 0 & 0 \\
-1 & 0 & 0 \\
0 & 2 & 0
\end{array}\right)
$$

A special type of subalgebra, called Cartan subalgebra, provides a crucial tool for the Cartan-Killing classification of simple Lie algebras. The formal definition needs two other concepts. A Lie algebra $\mathfrak{g}$ is called nilpotent if $\mathfrak{g}^{n}=0$ for some $n$, where $\mathfrak{g}^{0}:=\mathfrak{g}$ and $\mathfrak{g}^{i}:=\left[\mathfrak{g}^{i-1}, \mathfrak{g}\right]$ for $i \geq 1$. The normalizer of a subalgebra $\mathfrak{h}$ of a Lie algebra $\mathfrak{g}$ is defined as

$$
N_{\mathfrak{g}}(\mathfrak{h}):=\{g \in \mathfrak{g} \mid[g, \mathfrak{h}] \subset \mathfrak{h}\} .
$$

Definition 6. A Cartan subalgebra $\mathfrak{h} \subset \mathfrak{g}$ is a nilpotent subalgebra which is equal to its normalizer in $\mathfrak{g}$.

For simple finite-dimensional Lie algebras, a Cartan subalgebra is a maximal abelian subalgebra such that ad is diagonalizable for every of its elements (see [17, Chapter 8 and $15])$.

\footnotetext{
${ }^{1}$ It is no coincidence the name of these algebras with the groups $\mathrm{GL}_{n}(\mathbb{K})$ and $\mathrm{SL}_{n}(\mathbb{K})$. They are the Lie algebras of these Lie groups.
} 
Definition 7. Let $\mathfrak{g}$ be a Lie algebra with a Cartan subalgebra $\mathfrak{h}$ and $\alpha \in \mathfrak{h}^{*} \backslash\{0\}$ be a linear functional. If $\mathfrak{g}_{\alpha}:=\{g \in \mathfrak{g} \mid[h, g]=\alpha(h) g, h \in \mathfrak{h}\} \neq 0$, then $\alpha$ is called a root of $\mathfrak{g}$ and $\mathfrak{g}_{\alpha}$ a root space.

We know from linear algebra that when diagonalizable linear transformations commute then they are simultaneously diagonalizable. So given a Cartan subalgebra $\mathfrak{h}$ we can decompose $\mathfrak{g}$ as a direct sum of root spaces $\mathfrak{g}_{\alpha}$ and the centralizer of $\mathfrak{h}$ which is actually equal to $\mathfrak{h}$ (see [17, Proposition 8.2] ). This decomposition is known as the root decomposition or Cartan decomposition. The set of roots of a Lie algebra will be called root system and denoted by $\Phi$. So the Cartan decomposition is written as

$$
\mathfrak{g}=\mathfrak{h} \oplus \bigoplus_{\alpha \in \Phi} \mathfrak{g}_{\alpha}
$$

Remark. All Cartan subalgebras of a Lie algebra are conjugate, therefore the root system is uniquely determined up to an isomorphism (see [17, section 16]).

Example: Let us take the Lie algebra $\mathfrak{g}=\mathfrak{s l}_{2}(\mathbb{K})$ again and take $\mathfrak{h}=\langle h\rangle$. Since $\operatorname{ad}(h)$ is diagonal in the basis $\{e, h, f\}$, this is an ad diagonalizable subalgebra. It is also a maximal abelian algebra since $[h, \alpha e+\beta f]=0$ would imply $\alpha=\beta=0$. So $\mathfrak{h}$ is a Cartan subalgebra, and we have $\mathfrak{g}_{2}=\langle e\rangle$ and $\mathfrak{g}_{-2}=\langle f\rangle$. So the roots of $\mathfrak{s l}_{2}(\mathbb{K})$ are 2 and -2 , where we identify a linear operator in $\mathfrak{h}^{*}$ with its value on $h$.

Let $\mathfrak{g}$ be a Lie algebra and $g, h \in \mathfrak{g}$. We define $\kappa(g, h)=\operatorname{Tr}(\operatorname{ad} g$ ad $h)$. This symmetric bilinear form is known as the Killing form. For simple Lie algebras, this form is nondegenerate [17, Chapter 5]. Hence it will induce a bilinear form on the dual space $\mathfrak{g}^{*}$ and, in fact, by restriction, it will give us a bilinear form on the $\mathfrak{h}^{*}$. We shall denote this form by parenthesis.

Example: For any root $\alpha$ of a Lie algebra $\mathfrak{g}$, denote by $t_{\alpha}$ the element of $\mathfrak{g}$ such that $\alpha(g)=\kappa\left(t_{\alpha}, g\right)$. Let us take $\mathfrak{s l}_{2}$ with the usual basis $(e, h, f)$. We already have the adjoint matrices of these elements with respect to this basis. To compute the Killing form, it only remains to multiply them and take the trace. Then we obtain the following matrix for the Killing form:

$$
\kappa=\left(\begin{array}{lll}
0 & 0 & 4 \\
0 & 8 & 0 \\
4 & 0 & 0
\end{array}\right)
$$

whose determinant is -128 , so it is nondegenerate if char $\mathbb{K} \neq 2$.

The Lie algebra $\mathfrak{s l}_{2}$ has two roots $\alpha$ and $-\alpha$ where $\alpha(h)=2$. In order to construct the bilinear form on $\mathfrak{h}^{*}$, we need to find $t_{\alpha} \in \mathfrak{h}$ such that $\kappa\left(t_{\alpha}, h\right)=\alpha(h)=2$. Using the matrix of $\kappa$ it is straightforward to obtain $t_{\alpha}=h / 4$, so $(\alpha, \alpha)=\kappa\left(t_{\alpha}, t_{\alpha}\right)=1 / 2$.

It is more interesting to look at $\mathfrak{s l}_{3}$, this is an eight dimensional Lie algebra, the usual basis is the following:

$$
e_{1}=\left(\begin{array}{lll}
0 & 1 & 0 \\
0 & 0 & 0 \\
0 & 0 & 0
\end{array}\right), \quad e_{2}=\left(\begin{array}{lll}
0 & 0 & 0 \\
0 & 0 & 1 \\
0 & 0 & 0
\end{array}\right), \quad e_{3}=\left[e_{1}, e_{2}\right]=\left(\begin{array}{lll}
0 & 0 & 1 \\
0 & 0 & 0 \\
0 & 0 & 0
\end{array}\right)
$$




$$
\begin{array}{ccc}
f_{1}=\left(\begin{array}{lll}
0 & 0 & 0 \\
1 & 0 & 0 \\
0 & 0 & 0
\end{array}\right), \quad f_{2}=\left(\begin{array}{lll}
0 & 0 & 0 \\
0 & 0 & 0 \\
0 & 1 & 0
\end{array}\right), \quad f_{3}=\left[f_{2}, f_{1}\right]=\left(\begin{array}{lll}
0 & 0 & 0 \\
0 & 0 & 0 \\
1 & 0 & 0
\end{array}\right), \\
h_{1}=\left(\begin{array}{ccc}
1 & 0 & 0 \\
0 & -1 & 0 \\
0 & 0 & 0
\end{array}\right) & \text { and } \quad h_{2}=\left(\begin{array}{ccc}
0 & 0 & 0 \\
0 & 1 & 0 \\
0 & 0 & -1
\end{array}\right) .
\end{array}
$$

There are three copies of $\mathfrak{s l}_{2}$ inside of $\mathfrak{s l}_{3}$, indeed, $\left\langle e_{1}, h_{1}, f_{1}\right\rangle,\left\langle e_{2}, h_{2}, f_{2}\right\rangle$ and $\left\langle e_{3}, h_{1}+\right.$ $\left.h_{2}, f_{3}\right\rangle$ are all isomorphic to $\mathfrak{s l}_{2}$. If we take as Cartan subalgebra $\mathfrak{h}=\left\langle h_{1}, h_{2}\right\rangle$, we expect to have three pairs of roots that form root systems of $\mathfrak{s l}_{2}$. To complete the action of the Cartan subalgebra we need the following brackets: $\left[h_{i}, e_{j}\right]=-e_{j},\left[h_{i}, e_{3}\right]=e_{3},\left[h_{i}, f_{j}\right]=f_{j}$ and $\left[h_{i}, f_{3}\right]=-f_{3}$, where $1 \leq i \neq j \leq 2$.

Fixing a basis of $\mathfrak{s l}_{3}$ as $\left\{e_{1}, e_{2}, e_{3}, f_{1}, f_{2}, f_{3}, h_{1}, h_{2}\right\}$ we have $\operatorname{ad}\left(h_{1}\right)=\operatorname{diag}(2,-1,-2,1$, $1,-1,0,0)$ and $\operatorname{ad}\left(h_{2}\right)=\operatorname{diag}(-1,2,1,-2,1,-1,0,0)$. We can calculate the Killing form restricted to $\mathfrak{h}$ :

$$
\kappa_{\mathfrak{h}}=\left(\begin{array}{cc}
12 & -6 \\
-6 & 12
\end{array}\right)
$$

The roots are $\alpha=(2,-1), \beta=(-1,2), \alpha+\beta=(1,1)$ and their opposites $-\alpha,-\beta$, $-\alpha-\beta$, where we identified the roots by their values on $\left(h_{1}, h_{2}\right)$. From the Killing form we obtain $t_{\alpha}=h_{1} / 6, t_{\beta}=h_{2} / 6$ and $t_{\alpha+\beta}=\left(h_{1}+h_{2}\right) / 6$, which will imply $(\alpha, \alpha)=(\beta, \beta)=1 / 3$ and $(\alpha, \beta)=-1 / 6$. We can realize root systems as vectors in $\mathbb{R}^{\operatorname{dim}(\mathfrak{h})}$ and the bilinear form as the inner product. In the case of $\mathfrak{s l}_{3}$ all roots will have the same length and the angle $\theta$ between $\alpha$ and $\beta$, will be given by

$$
\cos \theta=\frac{(\alpha, \beta)}{\sqrt{(\alpha, \alpha)(\beta, \beta)}}=-\frac{1}{2},
$$

which implies $\theta=2 \pi / 3$, so we can represent the roots as follows:

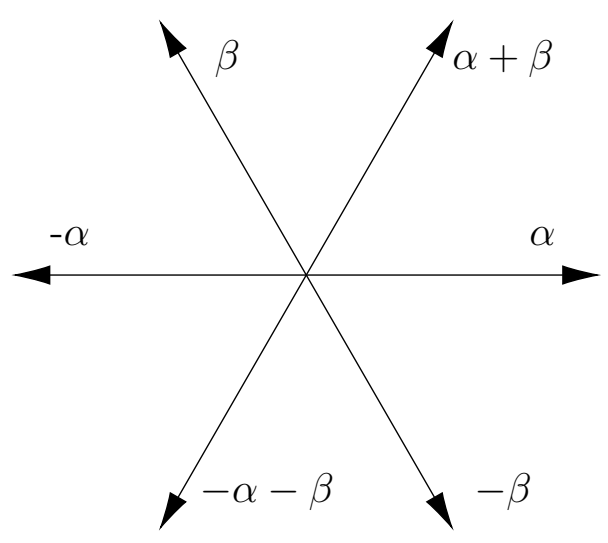

The following theorem summarizes the properties of a root system in general:

Theorem 8. [17, Theorem 8.4] Let $\mathfrak{g}$ be a Lie algebra, $\mathfrak{h}$ a Cartan subalgebra of $\mathfrak{g}$ and $\Phi$ the root system with respect to $\mathfrak{h}$. Then:

1. $\Phi$ spans $\mathfrak{h}^{*}$.

2. If $\alpha \in \Phi$ then $-\alpha \in \Phi$, but no other scalar multiple of $\alpha$ belongs to $\Phi$. 
3. If $\alpha, \beta \in \Phi$ then

$$
\langle\alpha, \beta\rangle:=\frac{2(\beta, \alpha)}{(\alpha, \alpha)}
$$

is an integer and $\alpha-\langle\alpha, \beta\rangle \beta$ belongs to $\Phi$.

These restrictions show us that the possibilities for root systems are very limited, for example, there exists only one possible root system of dimension one and only four of dimension 2. These are all the ingredients that one needs in order to classify all simple Lie algebras of finite dimensions.

Eugene Dynkin [12] gave a very elegant proof of the classification of simple Lie algebras of finite dimension using a diagram that today has the name Dynkin diagram in his honour. An important part of his classification was the definition of simple roots:

Definition 9. Let $\Phi$ be a root system. A subset $\Delta \subset \Phi$ is called a basis of $\Phi$ if every element of $\Phi$ can be uniquely written as a combination of elements in $\Delta$ with the coefficients all non-negative or all non-positive.

Given a root system, it is always possible to construct a basis, the elements of a basis are called simple roots. In our example of $\mathfrak{s l}_{3}, \alpha$ and $\beta$ could be chosen as simple roots. Given a basis we define a Cartan matrix as $A_{i j}:=\left\langle\alpha_{i}, \alpha_{j}\right\rangle$, where $\alpha_{i}, \alpha_{j}$ are simple roots. One important remark is that, up to reordering, a Lie algebra will only have one Cartan matrix. In other words, different Cartan subalgebras or a different choice of basis will give the same Cartan matrix, up to an ordering of the roots.

To each Cartan matrix, we associate a Dynkin diagram, which consists of a set of vertices and edges. The vertices are the simple roots and the number of edges between $\alpha_{i}, \alpha_{j}$ is the product $A_{i j} A_{j i}$. When these numbers are not equal the edges are marked with one arrow pointing to the shorter root. The classification of all possible diagrams can be found in [17, Chapter 11].

Example: For $\mathfrak{s l}_{2}$ we have only one simple root so the Cartan matrix will have only one entry equal to 2 and the Dynkin diagram will consist of only one vertex with no edges. For $\mathfrak{s l}_{3}$ we have $\langle\alpha, \beta\rangle=\langle\beta, \alpha\rangle=-1$, so the Cartan matrix is:

$$
\left(\begin{array}{cc}
2 & -1 \\
-1 & 2
\end{array}\right)
$$

and the Dynkin diagram will be:

Two questions arise. First, can two non-isomorphic Lie algebras generate the same root system? And, second, given a root system that satisfies the conditions of Theorem 8, will it generate a Lie algebra? Both questions are easily answered with the so-called Serre-Chevalley realization.

Let $\Delta=\left\{\alpha_{1}, \ldots, \alpha_{n}\right\}$ be a basis of a root system and let $A$ be its associtated Cartan matrix. Let $\mathfrak{h}=\operatorname{span}\left\{h_{1}, \ldots, h_{n}\right\}$ and define the action of $\Delta$ in $\mathfrak{h}$ as $\alpha_{j}\left(h_{i}\right)=A_{i j}$. Define the Lie algebra $\mathfrak{g}$ generated by the $3 n$ elements $\left\{e_{i}, h_{i}, f_{i}\right\}$ with $i=1, \ldots, n$ and the following relations:

1. $\left[h_{i}, h_{j}\right]=0$, 
2. $\left[e_{i}, f_{j}\right]=\delta_{i j} h_{i}$,

3. $\left[h_{i}, e_{j}\right]=A_{i j} e_{j}$,

4. $\left[h_{i}, f_{j}\right]=-A_{i j} f_{j}$,

5. $\operatorname{ad}\left(e_{i}\right)^{1-A_{i j}}\left(e_{j}\right)=0$,

6. $\operatorname{ad}\left(f_{i}\right)^{1-A_{i j}}\left(f_{j}\right)=0$.

Then $\mathfrak{g}$ will be a Lie algebra, $\mathfrak{h}$ will be a Cartan subalgebra of $\mathfrak{g}$, the root system of $\mathfrak{g}$ will be the one generated by $\Delta$. If the Cartan matrix is indecomposable then the Lie algebra will be simple. This construction shows us that for any Cartan matrix there exists a Lie algebra associated, and it is easy to construct an isomorphism from a Lie algebra with a Cartan algebra to the one generated by the Serre-Chavalley relations.

Observe that for each root $\alpha_{i}$ we have a copy of $\mathfrak{s l}_{2}$, since $A_{i i}=2$ for every $i$. The element $h_{i}$ obtained that way is called the co-root associated with $\alpha_{i}$. We can also express $h_{i}$ as

$$
\frac{2 t_{\alpha_{i}}}{\left(\alpha_{i}, \alpha_{i}\right)}
$$

Observe that this coincides with our definition:

$$
\alpha_{j}\left(h_{i}\right)=\frac{2 \alpha_{j}\left(t_{i}\right)}{\left(\alpha_{i}, \alpha_{i}\right)}=A_{i j}
$$

Given a basis of a root system, we will say that a root $\alpha$ is positive if all its coefficients in this basis are non-negative and we shall write $\alpha>0$, respectively, we say that $\alpha$ is negative if all its coefficients are non-positive and we shall write $\alpha<0$. Then the set of roots is divided in two parts, the positive roots and the negative roots. This will induce a decomposition of the Lie algebra known as the triangular decomposition. Let

$$
\mathfrak{n}_{+}:=\bigoplus_{\alpha>0} \mathfrak{g}_{\alpha} \text { and } \mathfrak{n}_{-}:=\bigoplus_{\alpha<0} \mathfrak{g}_{\alpha}
$$

Then we have

$$
\mathfrak{g}=\mathfrak{n}_{-} \oplus \mathfrak{h} \oplus \mathfrak{n}_{+}
$$

This decomposition will be important for us in the next section.

Example: Let $\mathfrak{g}=\mathfrak{s l}_{3}$ and let us the same notation of the previous example. We have

$$
\mathfrak{n}_{+}=\mathfrak{g}_{\alpha} \oplus \mathfrak{g}_{\beta} \oplus \mathfrak{g}_{\alpha+\beta}=\mathbb{K} e_{1} \oplus \mathbb{K} e_{2} \oplus \mathbb{K}\left[e_{1}, e_{2}\right] .
$$

Analogously, $f_{1}, f_{2}$ and $\left[f_{1}, f_{2}\right]$ will generate the negative part.

\subsection{Representations}

In the words of Pavel Etingof: "Very roughly speaking, representation theory studies symmetry in linear spaces."[13]. 
The idea behind the representation theory is to realize an algebraic structure as endomorphisms of vector spaces. When the vector space is finite-dimensional we can identify elements of an abstract algebraic structure with matrices. This gives us a new way to interpret a, sometimes, very hard structure in a much more tangible way. Not only that, the classification of all representations also gives us all the possible ways we can use the algebraic structure acting somewhere.

Observe that it seems we are talking about morphisms between different categories. Nevertheless, we can circumvent the restriction creating some associative algebra underlying our structure. For example, group representations are, in fact, representations of the group algebra. In the case of a Lie algebra, the corresponding associative algebra is the universal enveloping algebra, which will be presented at the second subsection. In the last subsection, we present their representations.

\subsubsection{Representations of associative algebras}

Definition 10. A representation of an associative algebra $A$ is a vector space $V$ together with a homomorphism of associative algebras $\rho: A \rightarrow \operatorname{End}(V)$.

Given a representation $(V, \rho)$ of an associative algebra $A$ we may also call $V$ a representation or a module for $A$. We also may omit $\rho$ when we talk about $A$ acting on $V$, writing $a v=\rho(a) v$ for $a \in A$ and $v \in V$.

Examples: Let $A$ be an associative algebra.

1. The space $V=\{0\}$ is a representation called the trivial representation.

2. If $A$ is 1-dimensional, that is, it is a field, then every representation is a vector space over $A$.

3. Let $V$ and $W$ be representations of $A$. Then $V \oplus W$ is a representation of $A$ with the action given by $a(v, w)=(a v, a w)$, for $a \in A, v \in V$ and $w \in W$.

Given $W \subset V$, if $A W:=\operatorname{Span}\{a w \mid a \in A, w \in W\} \subset W$, we say that $W$ is a subrepresentation of $V$. If $W$ is equal to 0 or $V$ then $W$ is called a trivial subrepresentation. If a representation $V$ contains only trivial subrepresentations, it is called irreducible and if it cannot be written as a direct sum of two non-trivial subrepresentation, it is called indecoponsable.

The main problem in representation theory is the classification of irreducible and indecomposable representations. An irreducible representation is indecomposable while the converse is not true. For finite groups the converse is also true, it is known as Maschke's theorem.

Example: Let $S_{n}$ be the group of permutation of $n$ elements. Then $\mathbb{K}^{n}$ is a representation with the action $\sigma\left(e_{i}\right)=e_{\sigma(i)}$, where $\sigma \in S_{n}$ and $\left\{e_{1}, \ldots, e_{n}\right\}$ is the standard basis of $\mathbb{K}^{n}$.

Let $V=\mathbb{K}\left(e_{1}+\ldots+e_{n}\right)$. Since every permutation acts on $V$ as identity, we have $S_{n} V=V$. So $V$ is a submodule, since it is 1-dimensional it is irreducible. The space $V^{\perp}$ of is also an irreducible submodule. This fact can be proven using character theory in 
a very simply way ${ }^{2}$. An interesting consequence is that given any two non-zero vectors with the sum of their coordinates equal to 0 , we can write one as a linear combination of permutations of the other.

Lemma 11 (Schur's lemma). Let $\rho_{1}: A \rightarrow \operatorname{End}\left(V_{1}\right)$ and $\rho_{2}: A \rightarrow \operatorname{End}\left(V_{2}\right)$ be two irreducible finite dimensional representations of $A$. Let $f: V_{1} \rightarrow V_{2}$ be a linear map such that $f \rho_{1}(a)=\rho_{2}(a) f$, for every $a \in A$. Then

1. $f=0$ if $\left(V_{1}, \rho_{1}\right)$ is not isomorphic to $\left(V_{2}, \rho_{2}\right)$.

2. $f$ is a multiplication by a scalar if $\left(V_{1}, \rho_{1}\right)=\left(V_{2}, \rho_{2}\right)$.

Proof. Although the proof is very similar for different structures, they are some idiosyncrasies. For groups see [30, Proposition 4]. For Lie algebras see [15, Theorem 4.29].

\subsubsection{Universal enveloping algebra}

To construct representations of Lie algebras, we need some underlying associative structure. One way is to map a Lie algebra $\mathfrak{g}$ into the Lie algebra $A^{(-)}$for some associative algebra $A$. The universal enveloping algebra for a Lie algebra will be an associative algebra with 1 , that can be considered containing all such maps as quotients. The formal definition is given as follows:

Definition 12. A universal enveloping algebra of a Lie algebra $\mathfrak{g}$ is an associative algebra $\mathcal{U}(\mathfrak{g})$ together with a Lie associative map $i: \mathfrak{g} \rightarrow \mathcal{U}(\mathfrak{g})$ such that the following holds: for any associative algebra $A$ and Lie associative map $j: \mathfrak{g} \rightarrow A$, there exists a unique homomorphism of associative algebras $\psi: \mathcal{U}(\mathfrak{g}) \rightarrow A$ such that the following diagram commutes:

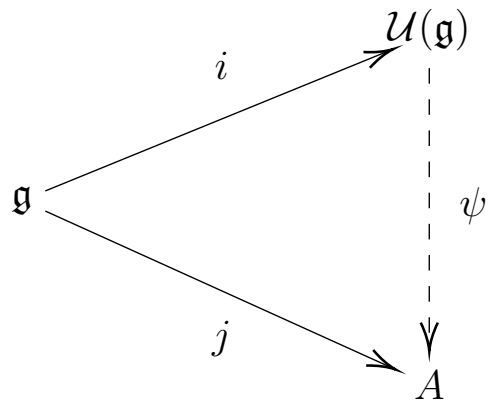

Two questions remain about this definition, does this object exist? And if it exists, is it unique? The second question is easier to answer. Suppose that for a given Lie algebra $\mathfrak{g}$ there exist two universal enveloping algebras $(\mathcal{U}(\mathfrak{g}), i)$ and $\left(U^{\prime}(\mathfrak{g}), i^{\prime}\right)$. If we set, in the previous diagram, $A=\mathcal{U}^{\prime}(\mathfrak{g})$ and $j=i^{\prime}$ we get $\psi: \mathcal{U}(\mathfrak{g}) \rightarrow \mathcal{U}^{\prime}(\mathfrak{g})$ and $\phi: \mathcal{U}^{\prime}(\mathfrak{g}) \rightarrow \mathcal{U}(\mathfrak{g})$. Now, if we set $A=\mathcal{U}(\mathfrak{g})$ and $j=i$ we get that the identity in $\mathcal{U}(g)$ and $\phi \circ \psi$ will work as the unique dotted map, so they must be the same. Analogously, $\psi \circ \phi$ and the identity in $\mathcal{U}^{\prime}(\mathfrak{g})$ are the same, so $\mathcal{U}(\mathfrak{g})$ is isomorphic to $\mathcal{U}^{\prime}(\mathfrak{g})$.

\footnotetext{
${ }^{2} \mathrm{An}$ excellent reference for this subject is Serre's book [30].
} 

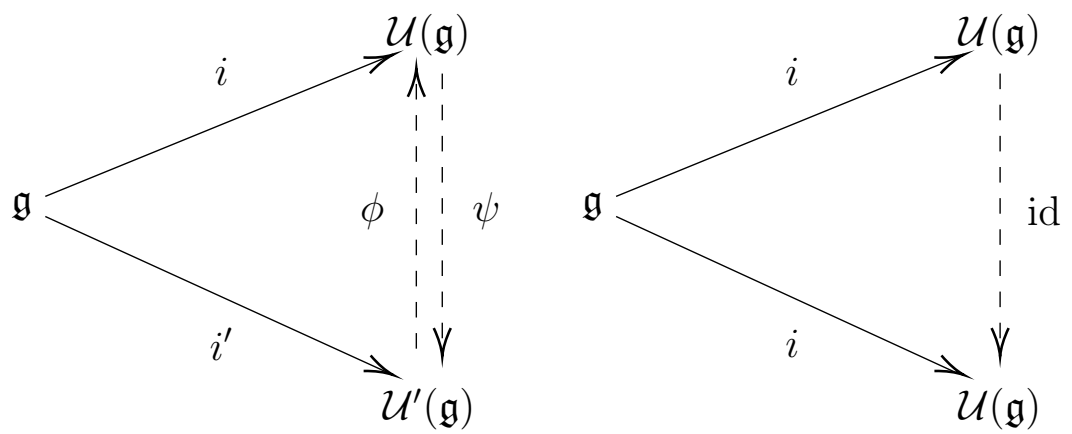

To answer the question about existence, we will give a construction of the universal enveloping algebra. Let $V$ be a $\mathbb{K}$-vector space and define $T^{0}(V)=\mathbb{K}, T^{1}(V)=V$, $T^{2}(V)=V \otimes V, \ldots$ and let

$$
T(V)=\bigoplus_{i=0}^{\infty} T^{i}(V) .
$$

A spanning set for $T(V)$ is given by elements of the form $v_{1} \otimes v_{2} \otimes \ldots \otimes v_{n}$, for arbitrary $n$. We can multiply elements in $T(V)$ in the following way

$$
\left(v_{1} \otimes \ldots \otimes v_{n}\right)\left(w_{1} \otimes \ldots \otimes w_{m}\right)=v_{1} \otimes \ldots \otimes v_{n} \otimes w_{1} \otimes \ldots \otimes w_{m},
$$

so $T^{n}(V) T^{m}(V) \subset T^{n+m}(V)$. This makes $T(V)$ a graded associative algebra which is called the tensor algebra. It is actually a universal object: given an associative algebra $A$ and a linear map $j: V \rightarrow A$ there exists a unique homomorphism of associative algebras $\psi: T(V) \rightarrow A$ such that $j=\psi \circ i$ where $i$ is the inclusion $i: V \rightarrow T(V)$.

To construct the universal enveloping algebra, we will need to take a quotient of the tensor algebra, so that the structure of Lie algebra is preserved. Before doing so, we will present two other important quotients of the tensor algebra as examples.

\section{Examples:}

1. Let $I_{S}$ be the two-sided ideal of $T(V)$ generated by all elements of the form $x \otimes y-$ $y \otimes x$ where $x, y \in V$. The quotient $S(V):=T(V) / I_{S}$, is called symmetric algebra. If we fix a basis for $V$ then $S_{n}(V)$ can be viewed as the polynomial algebra with the variables being the basis elements.

2. Take the two-sided ideal $I_{A}$ generated by all elements of the form $x \otimes y+y \otimes x$, then the quotient $\Lambda(V):=T(V) / I_{A}$, is called the exterior algebra. Unlike $S(V)$, the exterior algebra $\Lambda(V)$ is finite-dimensional, whenever $V$ is.

The universal enveloping algebra for a Lie algebra $\mathfrak{g}$ is constructed in a similar way as in the previous examples, in this case, we take the two-sided ideal $I_{U}$ in $T(\mathfrak{g})$, generated by all elements of the form $x \otimes y-y \otimes x-[x, y]$ where $x, y$ in $\mathfrak{g}$, then we define $\mathcal{U}(\mathfrak{g}):=T(\mathfrak{g}) / I_{U}$.

The proof of the universality of $\mathcal{U}(\mathfrak{g})$ follows from the universality of $T(\mathfrak{g})$. For any linear map $j: \mathfrak{g} \rightarrow A$ there is the unique $\psi: T(\mathfrak{g}) \rightarrow A$, but if $j$ is a Lie associative morphism then its kernel lies in $I_{U}$, so by the isomorphism theorem there exists a unique $\psi^{\prime}: \mathcal{U}(\mathfrak{g}) \rightarrow A$ such that the following diagram commutes: 


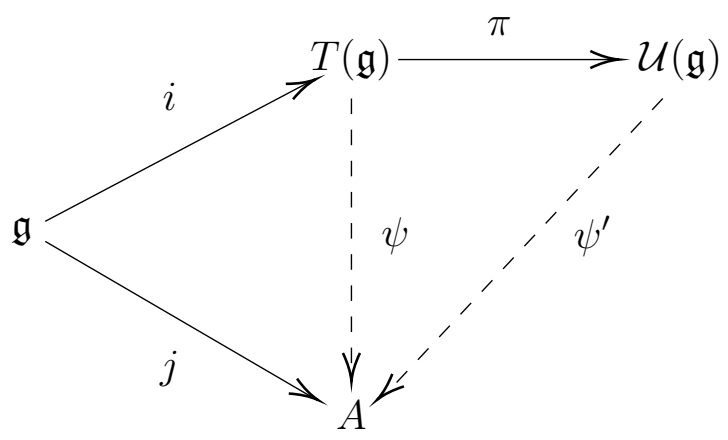

where $\pi: T(\mathfrak{g}) \rightarrow U(\mathfrak{g})$ is the projection.

Just from the definition of universal enveloping algebra it is very hard to describe the elements of it. However, there is one important theorem, named after Henri Poincaré, Garrett Birkhoff and Ernst Witt, which gives us a basis of the universal enveloping algebra:

Theorem 13. [17, Corollary $C$ of Theorem 17.3] Let $\mathfrak{g}$ be a Lie algebra and $\left(g_{1}, g_{2}, \ldots\right)$ an ordered basis of $\mathfrak{g}$. Then the elements $g_{i(1)} \ldots g_{i(m)}:=\pi\left(g_{i(1)} \otimes \ldots \otimes g_{i(m)}\right)$ with $m \in \mathbb{Z}^{+}$and $i(1) \leq \ldots \leq i(m)$ form a basis of $\mathcal{U}(\mathfrak{g})$. Here $\pi: T(\mathfrak{g}) \rightarrow \mathcal{U}(\mathfrak{g})$ is the natural projection.

This theorem is commonly referred to as PBW theorem. The following example shows how we can express an arbitrary element of the universal enveloping algebra in different forms and how to obtain the unique expression in terms of a basis given by the PBW theorem.

Example: Let us take the Lie algebra $\mathfrak{s l}_{2}(\mathbb{K})$ with the usual basis but with a different order $(f, h, e)$. Then a basis of $\mathcal{U}\left(\mathfrak{s l}_{2}(\mathbb{K})\right)$ is given by all $f^{n_{1}} h^{n_{2}} e^{n_{3}}$, with $n_{1}, n_{2}, n_{3} \in \mathbb{Z}^{+}$. For example, suppose we want to expand $e^{2} f$ in this basis. To do this we will have to proceed using commutators as follows:

$$
e^{2} f=e f e+e[e, f]=e f e+e h=f e^{2}+[e, f] e+h e+[e, h]=f e^{2}+2 h e-2 e .
$$

\subsubsection{Representations of Lie algebras}

Remember that from an associative algebra we can build a Lie algebra. Constructing a representation of a Lie algebra amounts to finding a copy of this Lie algebra or of its quotient in the set of endomorphisms of a vector space.

Hence a representation of a Lie algebra $\mathfrak{g}$ will consist of a vector space $V$ and a Lie associative map from $\mathfrak{g}$ to $\operatorname{End}(V)$. By the universal property of $\mathcal{U}(\mathfrak{g})$ this is equivalent to a representation of $\mathcal{U}(\mathfrak{g})$.

\section{Examples:}

1. For $\mathfrak{g l}_{n}$ there exists a representation, which is called natural representation, that consists of sending each matrix of $\mathfrak{g l}_{n}$ to the linear transformation given by that matrix in the canonical basis of $\mathbb{K}^{n}$. It is clear from this action that any non-zero 
vector $v \in \mathbb{K}^{n}$ will generate the whole space, so this is an example of an irreducible representation of $\mathfrak{g l}_{n}$.

2. Let $\mathfrak{g}$ be a Lie algebra and $V$ and $W$ be $\mathfrak{g}$-modules. Then $V \otimes W$ is a $\mathfrak{g}$-module with the action given by

$$
g(v \otimes w)=g v \otimes w+v \otimes g w,
$$

where $g \in \mathfrak{g}, v \in V$ and $w \in W$.

3. Let $V$ be a $\mathfrak{g}$-module, then the exterior algebra $\Lambda(V)$ is a $\mathfrak{g}$-module with the action given by:

$$
g\left(v_{1} \wedge \ldots \wedge v_{k}\right)=\sum_{i=1}^{k}\left(v_{1} \wedge \ldots \wedge g v_{i} \wedge \ldots \wedge v_{k}\right) .
$$

The subspaces $\Lambda^{k}(V)$ generated by elements of length $k$, that is, $v_{1} \wedge \ldots \wedge v_{k}$, are submodules of the exterior algebra and are called the $k$-th power of $V$. For $\mathfrak{g l}_{n}$ the powers of the natural module will play an important role in the next chapters.

For a given representation $(V, \rho)$ of a Lie algebra $\mathfrak{g}$, we can construct a dual representation on the dual space $V^{*}$. We define the dual representation $\rho^{*}: \mathfrak{g} \rightarrow \operatorname{End}\left(V^{*}\right)$ as

$$
\left(\rho^{*}(g) f\right)(v)=-f(\rho(g) v),
$$

where $f \in V^{*}$ and $v \in V$ and $g \in \mathfrak{g}$. The minus sign is needed to ensure that $\rho^{*}$ is a representation.

Given a module $V$ for a Lie algebra $\mathfrak{g}$ with a fixed Cartan subalgebra $\mathfrak{h}$ and $\alpha \in \mathfrak{h}^{*}$, define

$$
V_{\alpha}:=\{v \in V \mid h v=\alpha(h) v \text { for all } h \in \mathfrak{h}\},
$$

if $V_{\alpha} \neq 0$ we say that $\alpha$ is a weight of $V$ and $V_{\alpha}$ is a weight space. When $V$ is a direct sum of weight spaces we call $V$ a weight module.

Example: If $\mathfrak{g}$ is a simple finite-dimensional Lie algebra then $(\mathfrak{g}, \mathrm{ad})$ is a weight representation of $\mathfrak{g}$. The roots are the weights of this representation.

For simple finite-dimensional Lie algebras, every finite-dimensional representation must preserve the Jordan decomposition, in other words, it means that an ad diagonalizable element will also have its image, under the representation, also diagonalizable and every nilpotent element will have nilpotent action (see [17, Corollary 6.4]). This implies that every finite-dimension representation of a finite-dimensional Lie algebra is a weight representation.

Example: We will construct all irreducible finite-dimensional modules for $\mathfrak{s l}_{2}$. Let us start with an $\mathfrak{s l}_{2}$-weight module $M$. Let $v$ be an eigenvector of $h$ associated with the eigenvalue $\lambda$, that is, $h v=\lambda v$. We have

$$
h e v=e h v+[h, e] v=\lambda e v+2 e v=(\lambda+2) v
$$

and

$$
h f v=f h v+[h, f] v=\lambda f v-2 f v=(\lambda-2) v .
$$


So $e$ and $f$ act in $M$ by shifting the eigenvectors of $h$. Since we are looking for finitedimensional modules there must be some eigenvector on which $e$ will act as zero, otherwise, we could be generating infinitely many eigenvectors of $h$.

Let $\lambda$ be the eigenvalue of such eigenvector $v_{0}$, that is, $e v_{0}=0$ and $h v_{0}=\lambda v_{0}$. Let us construct a basis for $M$ by applying $f$ to $v_{0}: v_{1}:=f v_{0}, v_{2}:=f^{2} v_{0}$ and so on. In summary we have so far $h v_{n}=(\lambda-2 n) v_{n}$ and $f v_{n}=v_{n+1}$ for $n \in \mathbb{Z}^{+}$. It remains to calculate the action of $e$ on this set. We claim that $e v_{n}=(n \lambda-n(n-1)) v_{n-1}$. We will prove this by induction. For the basis of induction, we have $e v_{0}=0$. For the induction step suppose $e v_{n-1}=((n-1) \lambda-(n-1)(n-2)) v_{n-2}$, then we have

$$
\begin{aligned}
& e v_{n}=e f v_{n-1}=f e v_{n-1}+[e, f] v_{n-1}=f((n-1) \lambda-(n-1)(n-2)) v_{n-2}+h v_{n-1} \\
= & ((n-1) \lambda-(n-1)(n-2)+\lambda-2(n-1)) v_{n-1}=(n \lambda-n(n-1)) v_{n-1} .
\end{aligned}
$$

The whole action can be summarized in the following diagram:

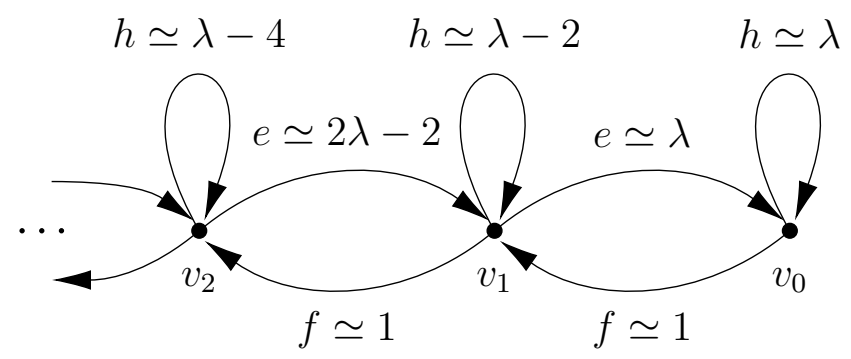

Here each arrow indicates the action of a basis element of $\mathfrak{s l}_{2}$. For example, it states that if you apply $e$ to $v_{1}$ you will obtain $v_{0}$ times $\lambda$, if you apply $h$ to $v_{2}$ you get $v_{2}$ times $\lambda-4$.

From this we actually got an infinite-dimensional module, however, if $\lambda=n \in \mathbb{Z}^{+}$, then we have that $e v_{n+1}=0$, so $v_{n+1}$ will generate a sub-module, let us call it $M^{\text {rad }}$. The quotient $L(\lambda):=M / M^{r a d}$ is our finite-dimensional module, actually any finite-dimensional module for $\mathfrak{s l}_{2}$ is isomorphic to some $L(\lambda)$ with $\lambda \in \mathbb{Z}^{+}$. For example, the trivial representation is $L(0)$, the natural representation is $L(1)$ and the adjoint representation is isomorphic to $L(2)$.

There is a generalization of this construction to any Lie algebra with a triangular decomposition, called the Verma module, in honour of Daya-Nand Verma.

Definition 14. Let $\mathfrak{g}$ be a Lie algebra with a triangular decomposition: $\mathfrak{n}_{-} \oplus \mathfrak{h} \oplus \mathfrak{n}_{+}$. Let $\lambda \in \mathfrak{h}^{*}$ and let $V$ be the one dimensional module for $\mathfrak{h} \oplus \mathfrak{n}_{+}$where $h v=\lambda(h) v$ for every $h \in \mathfrak{h}$ and $\mathfrak{n}_{+}$acts trivialy. Then the Verma module is defined as

$$
M(\lambda)=\mathcal{U}(\mathfrak{g}) \otimes_{\mathcal{U}\left(\mathfrak{h} \oplus \mathfrak{n}_{+}\right)} V .
$$

By the PBW theorem we have that

$$
M(\lambda) \simeq \mathcal{U}\left(\mathfrak{n}_{-}\right) \otimes_{\mathbb{K}} V
$$

A weight vector $v$ in a $\mathfrak{g}$-weight module $M$ such that the positive part of $\mathfrak{g}$ acts as zero is called a highest weight vector. If $M$ is generated by a highest weight vector it is called highest weigh module. Verma modules are highest weight modules by construction.

Verma modules are always infinite-dimensional weight modules. Except for $\mathfrak{s l}_{2}$, for all 
other simple finite-dimensional Lie algebras, the dimensions of the weight spaces are finite but not bounded.

For Verma modules, there is a simple condition to determine if they are irreducible. If $\lambda(h) \notin \mathbb{Z}^{+}$for all co-roots $h$, then $M(\lambda)$ is irreducible. For a proof of this fact see [17, Section 21.2] ${ }^{3}$.

Every Verma module contains a unique maximal submodule $M^{\text {rad }}$ and then $L(\lambda):=$ $M(\lambda) / M^{r a d}$ is irreducible. If $\lambda(h) \in \mathbb{Z}^{+}$for all co-roots, then $L(\lambda)$ is finite-dimensional. Every finite-dimensional irreducible module for simple finite-dimensional Lie algebras will be of this type.

Proposition 15. $\Lambda^{k}\left(\mathbb{K}^{N}\right)$ is an irreducible $\mathfrak{g l}_{N}$-module.

Proof. Let $\left\{e_{1}, \ldots, e_{N}\right\}$ be the standard basis of $\mathbb{K}^{N}$. Then $e_{a_{1}} \wedge \ldots \wedge e_{a_{k}}$ with $a_{1}<\ldots<a_{k}$ form a basis of $\Lambda^{k}\left(\mathbb{K}^{N}\right)$. Each element of this basis is a weight vector of distinct weight for the Cartan subalgebra $\mathfrak{h}=\left\langle E_{i i}\right\rangle$. This implies that every highest weight vector must be a multiple of some basis vector.

Let $e_{a_{1}} \wedge \ldots \wedge e_{a_{k}}$ be a highest weight vector, that is, $E_{i j}\left(e_{a_{1}} \wedge \ldots \wedge e_{a_{k}}\right)=0$ for $i<j$. For a fixed $E_{i j}$ the last equation is true if $a_{k}=i$ for some $k$ or none is equal to $j$. If we consider all pairs $(i, j)$ with $i<j$ the only possible vector that satisfies this equation is $e_{1} \wedge \ldots \wedge e_{k}$. Similar to the case of groups, for simple Lie algebras, indecoponsable representations will also be irreducible. This is know as Weyl's Theorem ([17][Theorem 6.3]). For our case, since there is only one highest weight vector and this module is finite-dimensional, we have that it is irreducible.

For $k \in\{1, \ldots, N\}$, the highest weights appearing in $\Lambda^{k}\left(\mathbb{K}^{N}\right)$ are special, they are called fundamental weights. Since

$$
E_{i i} e_{1} \wedge \ldots \wedge e_{k}=\left\{\begin{array}{lr}
e_{1} \wedge \ldots \wedge e_{k}, & i \in\{1, \ldots, k\} \\
0, & \text { otherwise }
\end{array}\right.
$$

we have that $h_{i}\left(e_{1} \wedge \ldots \wedge e_{k}\right)=\delta_{i k} e_{1} \wedge \ldots \wedge e_{k}$, where $h_{i}=E_{i i}-E_{i+1, i+1}$, is the usual set of co-roots. so these highest weights coincide with the dual basis of the set of co-roots.

Let $\mathfrak{g}$ be a Lie algebra, and let $Z(\mathcal{U}(\mathfrak{g}))$ be the center of the universal enveloping algebra. By Schur's Lemma 11 any element in $Z(\mathcal{U}(\mathfrak{g}))$ will act in an irreducible $\mathfrak{g}$-module by a scalar. Given a $\mathfrak{g}$-module $V$ we define $\chi: Z(\mathcal{U}(\mathfrak{g})) \rightarrow \mathbb{K}$ by taking each element of $Z(\mathcal{U}(\mathfrak{g}))$ into the scalar it is acting by. Such function is called a central character.

For some algebras, describing their center is not an easy task. For us the center of the universal enveloping algebra of $\mathfrak{g l}_{N}$ will be very important. Define the following elements of $U\left(\mathfrak{g l}_{N}\right)$ :

$$
\Omega_{k}=\sum_{i_{1}, \ldots, i_{k}=1}^{N} E_{i_{1} i_{2}} E_{i_{2} i_{3}} \cdots E_{i_{k} i_{1}}
$$

These are called Casimir elements. The Casimir elements give a description of $Z\left(\mathcal{U}\left(\mathfrak{g l}_{N}\right)\right)$ :

Proposition 16. [26, Corollary 7.1.2] $Z\left(\mathcal{U}\left(\mathfrak{g l}_{N}\right)\right)$ is a polynomial algebra in the variables $\Omega_{i}$ :

$$
Z\left(\mathcal{U}\left(\mathfrak{g l}_{N}\right)\right)=\mathbb{K}\left[\Omega_{1}, \ldots, \Omega_{N}\right]
$$

\footnotetext{
${ }^{3}$ In Humphrey's book Verma modules are called standard cyclic modules.
} 
Example: Note that $Z\left(\mathcal{U}\left(\mathfrak{g l}_{N}\right)\right)$ acts via a central character on any Verma module. In order to compute this central character we may apply the Casimirs to the highest weight vector. Let $\mathfrak{h}$ be the usual Cartan subalgebra of $\mathfrak{s l}_{N}$ and let $\lambda \in \mathfrak{h}^{*}$ and $\alpha \in \mathbb{K}$. Denote by $V(\lambda, \alpha)$ the $\mathfrak{g l}_{N}$-module which is the quotient $L(\lambda)$ of the Verma module $M(\lambda)$ for $\mathfrak{s l}_{N}$, and with the identity acting as multiplication by $\alpha$. So $\Omega_{1}=I d$ will act by $\alpha$.

Let us look at the case $N=2$. Let $v_{\lambda} \in V(\lambda, \alpha)$ be a maximal weight vector. We have that $\Omega_{2}$ will act on $v_{\lambda}$ as follows:

$$
\begin{aligned}
& \Omega_{2} v_{\lambda}=\left(E_{11}^{2}+E_{12} E_{21}+E_{21} E_{12}+E_{22}^{2}\right) v_{\lambda} \\
= & \left(\frac{1}{4}(\mathrm{Id}+h)^{2}+e f+f e+\frac{1}{4}(\mathrm{Id}-h)^{2}\right) v_{\lambda} \\
= & \left(\frac{1}{4}(\alpha+\lambda)^{2}+\lambda+\frac{1}{4}(\alpha-\lambda)^{2}\right) v_{\lambda}=\left(\frac{1}{2} \alpha^{2}+\frac{1}{2} \lambda^{2}+\lambda\right) v_{\lambda},
\end{aligned}
$$

which implies

$$
\lambda=-1 \pm \sqrt{1+\chi\left(\Omega_{2}\right)-\alpha}
$$

If we add the two possible values of $\lambda$ it gives us -2 . For a positive integer $n$, the only highest module with the same central character as $V(n, \alpha)$ is $V(-n-2, \alpha)$, which agrees with the construction of a Verma module $M(n)$. The first module is isomorphic to the quotient $L(n)$ and the second to the radical of $M(n)$.

Recall that every irreducible finite-dimensional module for $\mathfrak{g l}_{2}$ is a highest weight module. So the conclusion of this example is that given a central character there exists at most one irreducible finite-dimensional module with such central character.

For an arbitrary simple Lie algebra this result continues to be true, it is a consequence of an important theorem due to Harish-Chandra. To present his theorem let $\delta$ denote the half of the sum of all positive roots of $\mathfrak{g}$ and let $\mathcal{W}$ denote the group of symmetries of the root system of $\mathfrak{g}$, such group is called Weyl group named after Hermann Weyl. Let $\lambda \mu$ be weights, we will write $\lambda \simeq \mu$ if $\lambda+\delta$ is in the same orbit as $\mu+\delta$ under the action of the Weyl group.

Example: Let $\mathfrak{g}=\mathfrak{s l}_{2}$, we have two roots 2 and -2. So the Weyl group is isomorphic to $S_{2}$, the identity and the multiplication by -1 . In this case, $\delta$ is equal to 1 . For $\lambda$ and $\mu$ distinct weights of $\mathfrak{s l}_{2}$, we have $\lambda \simeq \mu$ if and only if $\lambda+1=-(\mu+1)$ which implies $\lambda+\mu=-2$ as we obtained in our previous example.

Theorem 17 (Harish-Chandra's isomorphism). Let $\mathfrak{g}$ be a simple Lie algebra with a Cartan subalgebra $\mathfrak{h}$. Let $\lambda, \mu \in \mathfrak{h}^{*}$. If $\chi_{\lambda}=\chi_{\mu}$ then $\lambda \simeq \mu$.

It follows from this theorem that there is at most one finite-dimensional simple module with a given central character. In order to properly address this question, we would have to discuss in much more detail the symmetries of root systems. However the general idea can be seen from our previous example. A finite-dimensional module will have the same character as modules in the same lattice obtained by a symmetry of the root system applied to the highest weight vector, this symmetry will switch the sign of at least one co-root. As we saw, the corresponding module will be infinite-dimensional. So, the central character defines uniquely a finite-dimensional module.

Example: Let us calculate central characters for $\Lambda^{0}\left(\mathbb{C}^{2}\right), \Lambda^{1}\left(\mathbb{C}^{2}\right), \Lambda^{2}\left(\mathbb{C}^{2}\right)$ as a $\mathfrak{g l}_{2}$ modules. For $\Lambda^{0}\left(\mathbb{C}^{2}\right)$, the central character is zero since the action is equal to zero for every element of $\mathfrak{g l}_{2}$. 
For $\Lambda^{1}\left(\mathbb{C}^{2}\right)$, we have that $\Omega_{1}$ will act as 1 , an the action of $\Omega_{2}$ can be calculated applying it to $e_{1}$ :

$$
\left(E_{11}^{2}+E_{12} E_{21}+E_{21} E_{12}+E_{22}^{2}\right) e_{1}=E_{11} e_{1}+E_{12} e_{2}=2 e_{1} .
$$

For $\Lambda^{2}\left(\mathbb{C}^{2}\right)$, we have $\Omega_{1}$ acting by 2 , an the action of $\Omega_{2}$ can be calculated applying it to $e_{1} \wedge e_{2}$ :

$$
\left(E_{11}^{2}+E_{12} E_{21}+E_{21} E_{12}+E_{22}^{2}\right)\left(e_{1} \wedge e_{2}\right)=\left(E_{11}+E_{22}\right)\left(e_{1} \wedge e_{2}\right)=2 e_{1} \wedge e_{2} .
$$

These central characters are presented in the following table:

\begin{tabular}{|l||l|l|l|}
\hline$\Omega_{1}$ & 0 & 1 & 2 \\
\hline$\Omega_{2}$ & 0 & 2 & 2 \\
\hline
\end{tabular}

So any finite-dimensional $\mathfrak{g l}_{2}$-module that has one of these characters will be isomorphic to one of the exterior powers of the natural module.

\subsection{Algebraic varieties}

The results in this section do not require that our field $\mathbb{K}$ is of characteristic zero. Let $\mathbb{A}_{\mathbb{K}}^{n}$ be the set of all $n$-tuples of elements of $\mathbb{K}$. For $f \in \mathbb{K}\left[x_{1}, \ldots, x_{n}\right]$, we can define a function from $\mathbb{A}_{\mathbb{K}}^{n}$ to $\mathbb{K}$ by evaluation: $\left(a_{1}, \ldots, a_{n}\right) \rightarrow f\left(a_{1}, \ldots, a_{n}\right)$ where $a_{1}, \ldots, a_{n} \in \mathbb{K}$. Let $I$ be an ideal of $\mathbb{K}\left[x_{1}, \ldots, x_{n}\right]$. Since $\mathbb{K}\left[x_{1}, \ldots, x_{n}\right]$ is a Noetherian ring, there exist $g_{1}, \ldots, g_{m}$ such that $I=\left\langle g_{1}, \ldots, g_{m}\right\rangle$. Since each $g_{i}$ is a polynomial, we can talk about theirs zeros. Let us denote the set of common zeros by

$$
V(I)=\left\{\left(a_{1}, \ldots, a_{n}\right) \in \mathbb{A}_{\mathbb{K}}^{n} \mid f\left(a_{1}, \ldots, a_{n}\right)=0 \text { for all } f \in I\right\} .
$$

Definition 18. A subset $X \in \mathbb{A}_{\mathbb{K}}^{n}$ is called an affine algebraic variety, or simply affine variety, if there exists ideal $I$ of $\mathbb{K}\left[x_{1}, \ldots, x_{n}\right]$ such that $X=V(I)$.

Proposition 19. The union of two affine varieties is an affine variety. The intersection of any family of affine varieties is an affine variety. The empty set and the whole space are affine varieties.

Proof. Let $X_{i}=V\left(I_{i}\right)$ be a family of affine varieties. Let $I_{i} I_{j}$ be the set of all linear combinations of polynomials that are a product of one polynomial in $I_{i}$ by one in $I_{j}$. Then $X_{i} \cup X_{j}=V\left(I_{i} I_{j}\right)$, indeed, any point in $X_{i} \cup X_{j}$ will be in $X_{i}$ or $X_{j}$ and it will be a zero of $I_{i}$ or $I_{j}$, respectively, in any case it will be a zero in $I_{i} I_{j}$. Now take a point $p$ in $V\left(I_{i} I_{j}\right)$ it must be a zero of $I_{i}$ or $I_{j}$, otherwise there would exist $f_{i} \in I_{i}$ and $f_{j} \in I_{j}$ such that $f_{i}(p) \neq 0 \neq f_{j}(p)$ and then $f_{i} f_{j}(p) \neq 0$. If $p$ is a zero in $I_{i}$ it belongs to $X_{i}$, if is a zero in $I_{j}$ it belongs to $X_{j}$.

The proof of $\bigcap X_{i}=V\left(\bigcup I_{i}\right)$ is analogous. Finally, we have $\mathbb{A}_{\mathbb{K}}^{n}=V(0)$ and $\emptyset=V(\langle 1\rangle)$.

From this proposition we can define a topology in $\mathbb{A}_{\mathbb{K}}^{n}$ where the affine varieties are the closed subsets, this is known as Zariski topology. 
Definition 20. A non-empty subset $X$ of a topological space $Y$ is irreducible if it cannot be expressed as a union $X=X_{1} \cup X_{2}$ of two proper Zariski closed subsets of $X$. The empty set is not considered to be irreducible.

Example: We have that $\mathbb{K}[x]$ is a principal ideal domain, so any affine variety in $\mathbb{A}_{\mathbb{K}}^{1}$ will be the set of zeros of a single polynomial. Since $\mathbb{A}_{\mathbb{K}}^{1}$ is infinite (our field is algebraically closed) and all proper affine varieties are finite, we have that $\mathbb{A}_{\mathbb{K}}^{1}$ is an irreducible variety.

For an affine variety $X \subset \mathcal{A}_{\mathbb{K}}^{n}$ we define

$$
\mathcal{I}(X):=\left\{f \in \mathbb{K}\left[x_{1}, \ldots, x_{n}\right] \mid f(X)=0\right\} .
$$

We have one functor that takes ideals into affine varieties and now one that takes affine varieties into ideals. The relations between these twp functors are shown by the following proposition:

Proposition 21. Let $X$ be any subset of $\mathcal{A}_{k}^{n}$ and $I$ an ideal in $\mathbb{K}\left[x_{1}, \ldots, x_{n}\right]$. Then

1. $V(\mathcal{I}(X))=\bar{X}$.

2. $\mathcal{I}(V(I))=\sqrt{I}$.

Here $\bar{X}$ denotes the closure of $X, i$. e., the smallest variety that contains $X$, and

$$
\sqrt{I}:=\left\{f \in \mathbb{K}\left[x_{1}, \ldots, x_{n}\right] \mid f^{r} \in I \text { for some } r \geq 1\right\}
$$

is called the radical of $I$.

The first part of this proposition follows from the fact that the zero set of an ideal is closed. The second part is a consequence of an important theorem known as Hilbert's Nullstellensatz:

Theorem 22 (Hilbert's Nullstellensatz). Let $I$ be an ideal of $\mathbb{K}\left[x_{1}, \ldots, x_{n}\right]$ and $f$ be $a$ polynomial that vanishes at every point of $V(I)$. Then $f^{r} \in I$ for some $r>0$.

For us, one corollary of this theorem will be important later in the text. But before stating the corollary we need the following definition:

Definition 23. Let $X$ be an affine variety and $I$ be an ideal of functions that vanish on $X$. Define the affine coordinate ring $\mathcal{A}(X)$ as $\mathbb{K}\left[x_{1}, \ldots, x_{n}\right] / I$.

For every element in $\mathcal{A}(X)$ one can define a function on $X$ by evaluation. So the coordinate ring is also called the ring of polynomial functions on $X$.

Corollary 24. Let $X$ be an affine variety and let $\left\{h_{i}\right\} \subset \mathcal{A}(X)$ be a set of polynomial functions on $X$ such that for all points $p \in X$ at least one of the $h_{i}$ is non-zero. Then the ideal generated by $\left\{h_{i}\right\}$ will be equal to the affine coordinate ring $\mathcal{A}(X)$.

Proof. Let $H$ be the ideal generated by $\left\{h_{i}\right\}$ and let $J \subset \mathbb{K}\left[x_{1}, \ldots, x_{N}\right]$ be the ideal given by the theorem of correspondence of ideals, i. e., the pre-image of $H$ under the projection on $\mathcal{A}(X)$. We have that $V(J) \cap X=\emptyset$ so, by the Hilbert's Nullstellensatz, the constant function on $X$ belongs to $J$ which implies that $\mathcal{A}(X) \subset H$. 
We define the dimension of an irreducible affine variety $X$ to be its dimension as a topological space, i.e., the supremum of all integers $n$ such that there exists a chain $Z_{0} \subset Z_{1} \subset \ldots \subset Z_{n}$ of distinct irreducible closed subsets of $X$.

The dimension of a ring is defined in a similar way, it is the supremum of integers $n$ such that there exists a chain $\mathfrak{p}_{0} \subset \mathfrak{p}_{1} \subset \ldots \subset \mathfrak{p}_{n}$ of distinct prime ideals.

Proposition 25. [16, Proposition 17] Let $X$ be an affine variety, then the dimension of $X$ is equal to the dimension of $\mathcal{A}(X)$.

In the remainder of this subsection we will discuss the notion of smoothness in algebraic varieties. From the geometric point of view, a variety is smooth if we can define a tangent space at its every point. Points where we cannot properly define a tangent space will be called singular points. Before introducing a formal definition, let us give some examples.

Examples: In the following examples we fix our field $\mathbb{K}=\mathbb{R}$ of the real numbers, even not being closed, it is the only one where we can draw, to give the idea of the types of singular points. In the next examples the origin will be a singularity, each of a different type.

1. The following graph is the variety $V\left(\left\langle x^{2}(x+1)-y^{2}\right\rangle\right)$. This type of a singular point is called a node. At this point you have more than one tangent line.

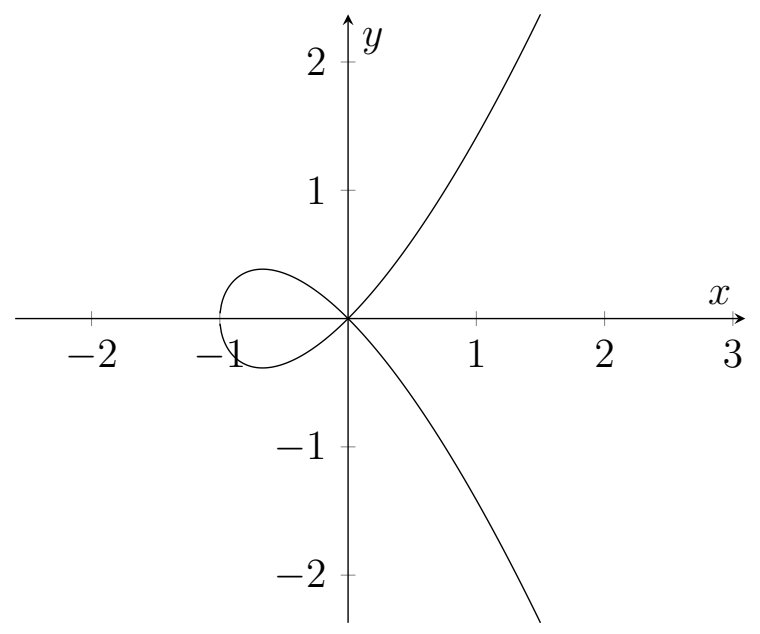

2. Another type of singular point is the one called cusp, here all partial derivatives vanish. An example of such phenomena is the variety $V\left(\left\langle x^{4}-x^{3}+y^{2}\right\rangle\right)$.

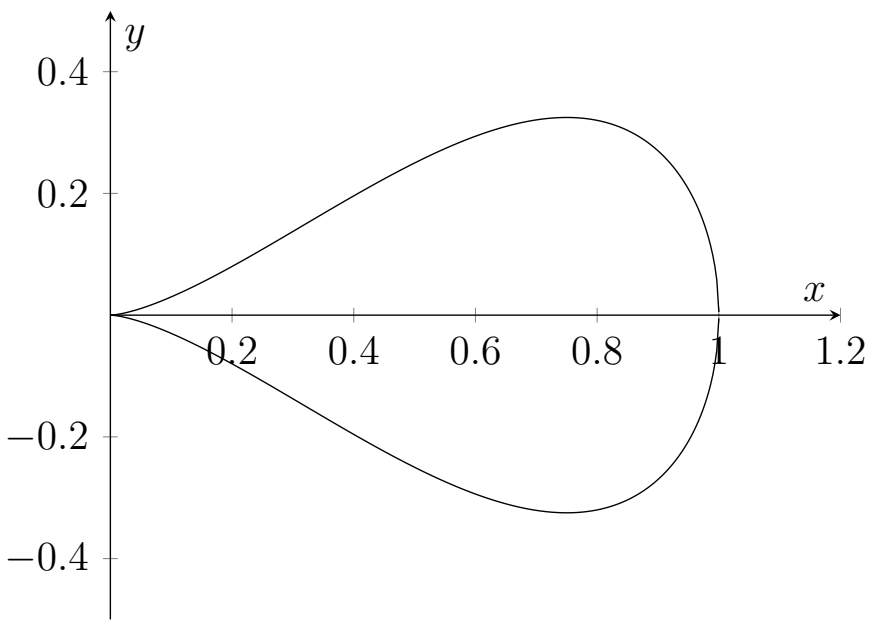


3. Consider the variety $V(\langle x y, x z\rangle) \subset \mathbb{R}^{3}$. This variety is a union of two other varieties the $y z$-plane, $V(x)$, and the $x$-axis, $V(y, z)$. As we mentioned before, the union of two varieties is a variety and can be obtained by taking the product of the corresponding ideals.

This is another type of singular point, the line would have one dimension and the plane two dimensions.

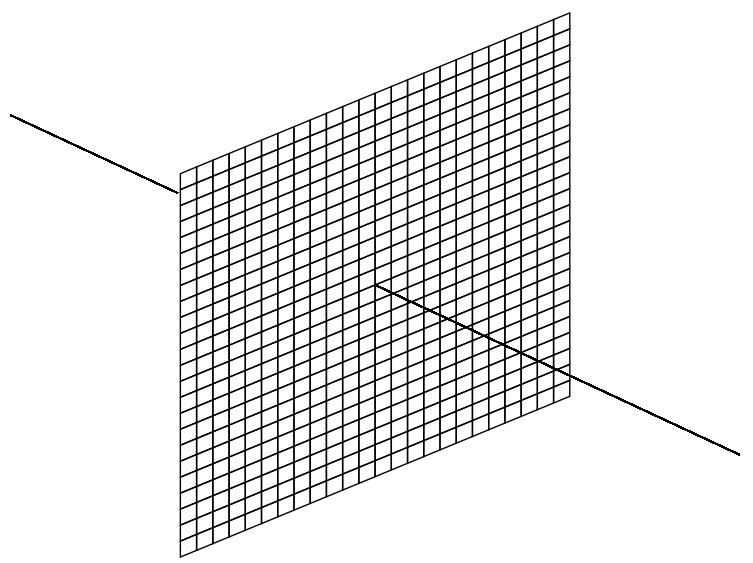

One way to define the tangent space is through the jacobian. It also helps to give a formal definition of singular points.

Definition 26. For a given variety $X \subset \mathcal{A}_{\mathbb{K}}^{n}$, Let $I=\left\langle g_{1}, \ldots, g_{m}\right\rangle$ be the ideal of functions that vanish on $X$. Then the $m \times n$ matrix

$$
J:=\left(\frac{\partial g_{i}}{\partial x_{j}}\right)_{i, j}
$$

is called the jacobian.

From this definition it is clear that $J$ depends on the choice of the generators of $I$. However, the rank of $J$ is independent of this choice (see [16][Theorem 5.1]). A point $p \in X$ is called singular if $\operatorname{rank} J(p)<n-\operatorname{dim} X$. A variety is called smooth if it does not contain singular points.

Example: Elliptic curves are plane curves given by $V\left(x^{3}+a x+b-y^{2}\right)$, where $a$ and $b$ are two parameters of the field, such that the curve is smooth. To decide the possible values of the parameters let us calculate the jacobian:

$$
J=\left(\begin{array}{ll}
3 x^{2}+a & 2 y
\end{array}\right)
$$

The rank of the jacobian is not 1 only when both expressions are equal to 0 . If we plug the equalities $a=-3 x^{2}$ and $y=0$ into the equation of the curve we obtain $-2 x^{3}+b=0$. So this equation does not define an elliptic curve if and only if $4 a^{3}+27 b^{2}=0$.

\section{$2.4 \mathcal{A} \mathcal{V}-$ modules}

As we have mentioned before, our objects of study will be modules which admit the action of both the Lie algebra of vector fields and the ring of polynomial functions on a 
smooth irreducible variety. The main idea of this section is to present a category where those modules lie.

\subsubsection{Charts}

Throughout this section, let our field $\mathbb{K}$ be an algebraically closed field of characteristic 0 , and let $X \subset \mathbb{A}_{\mathbb{K}}^{n}$ be a smooth irreducible affine variety of dimension $N$. Let $I=$ $\left\langle g_{1}, \ldots, g_{m}\right\rangle$ be the ideal of functions in $\mathbb{K}\left[x_{1}, \ldots, x_{n}\right]$ that vanish on $X$ and let $J$ be the jacobian matrix and $r$ its rank. Let $\mathcal{A}=\mathbb{K}\left[x_{1}, \ldots, x_{n}\right] / I$ be the algebra of polynomial functions on $X$, and let $\mathcal{V}=\operatorname{Der}(\mathcal{A})$ be the Lie algebra of vector fields on $X$. For the special case $X=\mathbb{A}^{N}$ we shall write $\mathcal{L}$ for the algebra of vector fields in $X$ :

$$
\mathcal{L}=\bigoplus_{i=1}^{N} \mathbb{K}\left[x_{1}, \ldots, x_{N}\right] \frac{\partial}{\partial x_{i}}
$$

If $Q$ is a monomial of degree $d$, we define the degree of the derivation $Q \frac{\partial}{\partial x_{i}}$ to be $d-1$. For $l \geq-1$, let $\mathcal{L}(l)$ the subalgebra of $\mathcal{L}$ consisting of derivations with no terms of degree less than $l$ and we have a filtration

$$
\mathcal{L} \supset \mathcal{L}(0) \supset \mathcal{L}(1) \supset \mathcal{L}(2) \supset \ldots
$$

Let $\left\{h_{i}\right\}$ be the set of non-zero $r \times r$ minors of $J$ and let $C\left(h_{i}\right):=\left\{p \in X \mid h_{i}(p) \neq 0\right\}$. The jacobian criterion for smoothness (see [16, Section I.5]) states that $\bigcup_{i} C\left(h_{i}\right)=X$. One way to describe the Lie algebra $\mathcal{V}$ is as an $\mathcal{A}$-submodule in the free $\mathcal{A}$-module $\oplus_{i=1}^{n} \mathcal{A} \frac{\partial}{\partial x_{i}}$, which is the kernel of the jacobian matrix: $\sum_{i=1}^{n} f_{i} \frac{\partial}{\partial x_{i}} \in \mathcal{V}$ if and only if

$$
\sum_{i=1}^{n} f_{i} \frac{\partial g_{j}}{\partial x_{i}}=0
$$

in $\mathcal{A}$ for all $j=1, \ldots, m$ (see e.g. [6]).

Fix a non-zero minor $h$ of $J$, let $\beta \subset\{1, \ldots, n\}$ be the set of corresponding columns of $J$ in $h$. For each $i \notin \beta$, we can find $f_{i j} \in h^{-1} \mathcal{A}$, such that $J \tau_{i}=0$ where

$$
\tau_{i}:=\frac{\partial}{\partial x_{i}}+\sum_{j \in \beta} f_{i j} \frac{\partial}{\partial x_{j}} .
$$

This is done solving the system $J f=0$. Since the rank of $J$ is equal to $|\beta|$, we can solve this system of linear equations treating $f_{i}, i \notin \beta$ as free variables.

Since $f_{i j} \in h^{-1} \mathcal{A}$ we have that $h \tau_{i} \in \operatorname{Der}(\mathcal{A})$. Note that each $\tau_{i}$ is a derivation of the localized algebra $\mathcal{A}_{(h)}$ but not necessarily of $\mathcal{A}$.

We have the following definition from [8]:

Definition 27. We shall say that $t_{1}, \ldots, t_{N} \in \mathcal{A}$ are chart parameters in the chart $C(h)$ provided that the following conditions are satisfied:

1. $t_{1}, \ldots, t_{N}$ are algebraically independent, so $\mathbb{K}\left[t_{1}, \ldots, t_{N}\right] \subset \mathcal{A}$.

2. Each element of $\mathcal{A}$ is algebraic over $\mathbb{K}\left[t_{1}, \ldots, t_{N}\right]$.

3. For each $i=1, \ldots, N$, the derivation $\frac{\partial}{\partial t_{i}} \in \operatorname{Der}\left(\mathbb{K}\left[t_{1}, \ldots, t_{N}\right]\right)$ extends to a derivation of the localized algebra $\mathcal{A}_{(h)}$. 
From this definition, the existence of chart parameters is not guaranteed. The next lemma gives us an example independent of charts and the variety, which assures the existence of chart parameters in any case:

Lemma 28. [8, Lemma 4] We have that $\left\{x_{i} \mid i \notin \beta\right\}$ are chart parameters in the chart $C(h)$.

It follows from the definition of chart parameters that

$$
\operatorname{Der}\left(\mathcal{A}_{(h)}\right)=\bigoplus_{i=1}^{N} \mathcal{A}_{(h)} \frac{\partial}{\partial t_{i}}
$$

Since $\operatorname{Der}(\mathcal{A}) \subset \operatorname{Der}\left(\mathcal{A}_{(h)}\right)$, each polynomial vector field $\eta$ on $X$ can be written as

$$
\eta=\sum_{i=1}^{N} f_{i} \frac{\partial}{\partial t_{i}}
$$

where $f_{i}=\eta\left(t_{i}\right) \in \mathcal{A}$. Note that here $\frac{\partial}{\partial t_{i}}$ is interpreted as the unique extension of the partial derivative on $\mathbb{K}\left[t_{1}, \ldots, t_{N}\right]$ to a derivation of $\mathcal{A}_{(h)}$.

In the case when we take $\left\{x_{i} \mid i \notin \beta\right\}$ as chart parameters, a derivation

$$
\eta=\sum_{i \notin \beta} f_{i} \frac{\partial}{\partial x_{i}}+\sum_{j \in \beta} f_{j} \frac{\partial}{\partial x_{j}}
$$

when embedded in $\operatorname{Der}\left(\mathcal{A}_{(h)}\right)$ will be written simply as

$$
\eta=\sum_{i \notin \beta} f_{i} \frac{\partial}{\partial x_{i}}
$$

with the understanding that for $j \in \beta$ we have $\eta\left(x_{j}\right)=\sum_{i \notin \beta} f_{i} \partial x_{j} / \partial x_{i}=\sum_{i \notin \beta} f_{i} \tau_{i}\left(x_{j}\right)$. With this convention we have $h \frac{\partial}{\partial x_{i}} \in \mathcal{V}$ for every $i \notin \beta$.

Example: Let us take $X$ to be a 2-dimensional sphere $\mathbb{S}^{2} \subset \mathbb{A}^{3}$ with a defining ideal $I=\left\langle x^{2}+y^{2}+z^{2}-1\right\rangle$. Then the jacobian matrix is

$$
J=\left(\begin{array}{lll}
2 x & 2 y & 2 z
\end{array}\right) .
$$

We have three minors $h_{1}=x, h_{2}=y$ and $h_{3}=z$. Each corresponding chart is the sphere without a great circle $x=0, y=0$ or $z=0$, respectively. Each point of $X$ belongs to at least one chart, which agrees with the fact that $\mathbb{S}^{2}$ is a smooth variety. Let us fix $h=z$ so that $x$ and $y$ are chart parameters. To find how the partial derivative $\frac{\partial}{\partial x}$ of $\mathbb{K}[x, y]$ extends to a derivation of $\mathcal{A}_{(z)}$ we need to solve the following equation

$$
J \tau_{x}=J\left(\begin{array}{c}
1 \\
0 \\
f_{x z}
\end{array}\right)=0,
$$

where $f_{x z} \in z^{-1} \mathbb{K}[x, y]$. Solving this equation, we obtain $f_{x z}=-x / z$, which implies

$$
\tau_{x}=\frac{\partial}{\partial x}-\frac{x}{z} \frac{\partial}{\partial z} .
$$


If we multiply $\tau_{x}$ by $h=z$ we obtain

$$
z \frac{\partial}{\partial x}-x \frac{\partial}{\partial z}
$$

which is a known vector field on $X$. Treating $z$ as an implicit function of $x$ and $y$, we will write $\tau_{x}=\frac{\partial}{\partial x}$ with understanding that

$$
\frac{\partial z}{\partial x}=-\frac{x}{z}
$$

Given a standard chart $C(h)$, fix a point $p$ in this chart and let $t_{1}, \ldots, t_{N}$ be the standard chart parameters centered at $p$, i.e. $t_{1}(p)=\cdots=t_{N}(p)=0$. One possible way to obtain such chart parameters is taking $t_{j}=x_{i_{j}}-p_{i_{j}}, i_{j} \notin \beta$. Write $\mathfrak{m}_{p}$ for the maximal ideal in $\mathcal{A}$ consisting of functions that vanish at $p$. For $l \geq-1$, define $\mathcal{V}(l):=\left\{\eta \in \mathcal{V} \mid \eta(\mathcal{A}) \in m_{p}^{l+1}\right\}$. Then we have a filtration of subalgebras

$$
\mathcal{V}=\mathcal{V}(-1) \supset \mathcal{V}(0) \supset \mathcal{V}(1) \supset \ldots,
$$

with $[\mathcal{V}(l), \mathcal{V}(k)] \subset \mathcal{V}(l+k)$ for $l+k \geq-1$. To simplify notation we shall sometimes write $\mathcal{V}_{+}$for $\mathcal{V}(0)$

Just from this definition, it is not easy to describe this filtration for most of the varieties. The easiest case is taking $X$ to be the affine space and $p$ equals the origin. We actually already did this at the beginning of this section. The next lemma gives an explicit description of this filtration.

Lemma 29. [7, Lemmas 5,6] (a) We have $\mathcal{V}(l)=m_{p}^{l+1} \mathcal{V}$.

(b) For any element $\mu \in \mathcal{L}$ whose terms all have degree less than $l$, there exists $\eta \in \mathcal{V}$ such that

$$
\eta=\mu+\text { terms of degree } \geq l .
$$

(c) There is an isomorphism of Lie algebras

$$
\mathcal{V}_{+} / \mathcal{V}(l) \simeq \mathcal{L}_{+} / \mathcal{L}(l)
$$

One isomorphism that is particularly important for us is $\rho: \mathcal{L}_{+} / \mathcal{L}(1) \rightarrow \mathfrak{g l}_{N}$ given by $\rho\left(t_{i} \partial / \partial t_{j}\right)=E_{i j}$. This is clearly a bijection. It is a homomorphism of Lie algebras, indeed,

$$
\begin{gathered}
\rho\left[t_{i} \frac{\partial}{\partial t_{j}}, t_{k} \frac{\partial}{\partial t_{l}}\right]=\rho\left(\delta_{j k} t_{i} \frac{\partial}{\partial t_{l}}-\delta_{i l} t_{k} \frac{\partial}{\partial t_{j}}\right) \\
=\delta_{j k} E_{i l}-\delta_{i l} E_{k j}=\left[E_{i j}, E_{k l}\right]=\left[\rho\left(t_{i} \frac{\partial}{\partial t_{j}}\right), \rho\left(t_{k} \frac{\partial}{\partial t_{l}}\right)\right] .
\end{gathered}
$$

Im particular, applying the previous lemma, we have $\mathcal{V}_{+} / \mathcal{V}(1) \simeq \mathfrak{g l}_{N}$. This isomorphism will be used in the construction of our modules.

Definition 30. An $\mathcal{A} \mathcal{V}$-module is a vector space $M$ equipped with module structures over both the commutative unital algebra $\mathcal{A}$ and over the Lie algebra $\mathcal{V}$ such that these structures are compatible in the following sense:

$$
\eta \cdot(f \cdot m)=\eta(f) \cdot m+f \cdot(\eta \cdot m)
$$


for all $\eta \in \mathcal{V}, f \in \mathcal{A}$, and $m \in M$.

We say that an $\mathcal{A} \mathcal{V}$-module $M$ has finite rank if it is finitely generated as an $\mathcal{A}$-module.

Another important construction for us is the Hopf smash product. Let $U$ be a Hopf algebra with co-product: $\Delta: U \rightarrow U \otimes U$. Write $\Delta(u)=\sum_{i} u_{i}^{1} \otimes u_{i}^{2}$. Let $A$ be an associative algebra that is a module for $\mathrm{U}$.

Definition 31. The smash product $A \# U$ is defined as the vector space $A \otimes U$ with the multiplication given as

$$
(a \otimes u)(b \otimes v)=\sum_{i} a u_{i}^{1}(b) \otimes u_{i}^{2} v .
$$

Recall that $\mathcal{A}$ is a $\mathcal{V}$-module with the action being the derivation. Identify $\mathcal{A}$ and $\mathcal{V}$ with their copies in $\mathcal{A} \# \mathcal{U}(\mathcal{V})$. The $\mathcal{A} \mathcal{V}$-module structure is equivalent to $\mathcal{A} \# \mathcal{U}(\mathcal{V})$-module structure. This structure will be important when we talk about Rudakov modules. 


\section{Chapter 3}

\section{Gauge Modules}

Tensor modules form an important class and have their origins in differential geometry. In [25] Mathieu proved that simple weight modules with finite-dimensional weight spaces over the Lie algebra of vector fields on the circle fall into two classes: highest weight modules or tensor modules. Tensor modules will be discussed in the first section of this chapter.

Motivated by non-abelian gauge theory, in [7] Billig, Futorny and Nilsson generalized tensor modules by including into the action gauge fields. These new modules are called gauge modules. When $X=\mathbb{S}^{1}$, gauge modules with non-scalar gauge fields are examples of non-weight modules.

Throughout this chapter we fix the following notation. Let $X$ be an affine smooth irreducible variety of dimension $N, \mathcal{A}$ its coordinate ring and $\mathcal{V}$ the Lie algebra of vector fields on $X$. We also fix the letter $h$ for non-zero minors of the jacobian matrix, $C(h)$ for the charts and $\left\{t_{1}, \ldots, t_{N}\right\}$ a set of chart parameters. When $X$ is the affine space $\mathbb{A}_{\mathbb{K}}^{N}$ we will write the Lie algebra of vector fields as $\mathcal{L}=\operatorname{Der}\left(\mathbb{K}\left[t_{1}, \ldots, t_{N}\right]\right)$, recall its natural $\mathbb{Z}$-grading: $\mathcal{L}=\mathcal{L}_{-1} \oplus \mathcal{L}_{0} \oplus \mathcal{L}_{1} \oplus \mathcal{L}_{2} \oplus \ldots$. We set $\mathcal{L}_{+}$to be a subalgebra of $\mathcal{L}$ of elements of non-negative degree: $\mathcal{L}_{+}=\mathcal{L}_{0} \oplus \mathcal{L}_{1} \oplus \mathcal{L}_{2} \oplus \ldots$ Note that $\mathcal{L}_{0}$ is spanned by the elements $t_{i} \frac{\partial}{\partial t_{j}}$ and it is isomorphic to $\mathfrak{g l}_{N}$. Refer to section 2.4 for more details about these structures.

\subsection{Tensor modules}

Although tensor modules may be defined for any variety, here we will define tensor modules only for the $N$-dimensional torus. This will be done in order to present another realization of the vector fields on an $N$-dimensional torus and, also, to simplify our notation and to present a theorem due to Rao.

One of the many problems in working with arbitrary varieties is to find good ways to present their elements. The presentation via charts solves this problem. Without this technique, only some varieties could be studied using some machinery that, most of the time, was only suitable for it.

For example, functions on the $N$-dimensional torus may be expressed via the variables $t_{j}=e^{i x_{j}}, j=1, \ldots, N$, where $i \in \mathbb{C}$ is the imaginary unit. The Lie algebra of vector fields on a torus then becomes

$$
\mathcal{V}_{\mathbb{T}^{N}}=\operatorname{Der}\left(\mathbb{C}\left[t_{1}^{ \pm 1}, \ldots, t_{N}^{ \pm 1}\right]\right)=\bigoplus_{p=1}^{N} \mathbb{C}\left[t_{1}^{ \pm 1}, \ldots, t_{N}^{ \pm 1}\right] \frac{\partial}{\partial t_{p}}
$$


It will be more convenient for us to work with the degree derivations $d_{p}=t_{p} \frac{\partial}{\partial t_{p}}$ as the free generators of $\mathcal{V}_{\mathbb{T}^{N}}$ as a $\mathbb{C}\left[t_{1}^{ \pm 1}, \ldots, t_{N}^{ \pm 1}\right]$-module:

$$
\mathcal{V}_{\mathbb{T}^{N}}=\bigoplus_{p=1}^{N} \mathbb{C}\left[t_{1}^{ \pm 1}, \ldots, t_{N}^{ \pm 1}\right] d_{p}
$$

The Lie bracket in $\mathcal{V}_{\mathbb{T}^{N}}$ is then written as

$$
\left[t^{r} d_{a}, t^{m} d_{b}\right]=m_{a} t^{r+m} d_{b}-r_{b} t^{r+m} d_{a}
$$

$a, b=1, \ldots, N$. Here we are using the multi-index notations $t^{r}=t_{1}^{r_{1}} \ldots t_{N}^{r_{N}}$ for $r=$ $\left(r_{1}, \ldots, r_{N}\right) \in \mathbb{Z}^{N}$.

Fix a finite-dimensional irreducible $\mathfrak{g l}_{N}$-module $U$. Let $\gamma \in \mathbb{C}^{N}$, this will be a shift in $\mathcal{A}$. We define the tensor module $T=T(U, \gamma)$ to be the vector space

$$
T=q^{\gamma} \mathbb{C}\left[q_{1}^{ \pm 1}, \ldots, q_{N}^{ \pm 1}\right] \otimes U
$$

with the action given by

$$
t^{r} d_{a}\left(q^{\mu} \otimes u\right)=\mu_{a} q^{\mu+r} \otimes u+\sum_{p=1}^{N} r_{p} q^{\mu+r} \otimes E_{p a} u,
$$

where $r \in \mathbb{Z}^{N}, \mu \in \gamma+\mathbb{Z}^{N}, a=1, \ldots, N$.

Theorem 32. [28, Theorem 1.9] Let $U$ be an irreducible finite-dimensional $\mathfrak{g l}_{N}$-module. The tensor module $T(U, \gamma)$ is an irreducible $\mathcal{V}_{\mathbb{T}^{N}}$-module, unless it appears in the de Rham complex of differential forms

$$
q^{\gamma} \Omega^{0}\left(\mathbb{T}^{N}\right) \rightarrow q^{\gamma} \Omega^{1}\left(\mathbb{T}^{N}\right) \rightarrow \ldots \rightarrow q^{\gamma} \Omega^{N}\left(\mathbb{T}^{N}\right) .
$$

The middle terms in this complex are reducible $\mathcal{V}_{\mathbb{T}^{N}}$-modules, while the terms $q^{\gamma} \Omega^{0}\left(\mathbb{T}^{N}\right)$ and $q^{\gamma} \Omega^{N}\left(\mathbb{T}^{N}\right)$ are reducible whenever $\gamma \in \mathbb{Z}^{N}$.

The tensor modules that appear in the de Rham complex correspond to the ones with $U$ as one of the exterior powers of the natural module $\Lambda^{k}\left(\mathbb{C}^{N}\right)$.

\subsection{Gauge fields}

Gauge modules are a generalization of tensor modules. The idea is to include in the action of the vector fields a differential 1-form on the variety, the components of this 1form will be called gauge fields. In order to keep the action they must satisfy the following axioms:

Definition 33. Let $(U, \rho)$ be a finite-dimensional $\mathcal{L}_{+}$-module.

Functions $B_{i}: \mathcal{A}_{(h)} \otimes U \rightarrow \mathcal{A}_{(h)} \otimes U, i=1, \ldots, N$, are called gauge fields if

1. each $B_{i}$ is $\mathcal{A}_{(h)}$-linear,

2. $\left[B_{i}, \rho\left(\mathcal{L}_{+}\right)\right]=0$,

3. $\left[\frac{\partial}{\partial t_{i}}+B_{i}, \frac{\partial}{\partial t_{j}}+B_{j}\right]=0$ as operators on $\mathcal{A}_{(h)} \otimes U$ for all $i, j \in\{1, \ldots, N\}$. 
Let $\left\{B_{i}\right\}$ be gauge fields, $i=1, \ldots, N$. Then the space $\mathcal{A}_{(h)} \otimes U$ is a $\operatorname{Der}\left(\mathcal{A}_{(h)}\right)$-module with the following action

$$
\left(f \frac{\partial}{\partial t_{i}}\right) \cdot(g \otimes u)=f \frac{\partial g}{\partial t_{i}} \otimes u+f g \otimes B_{i} u+\sum_{k \in \mathbb{Z}_{+}^{N} \backslash\{0\}} \frac{1}{k !} g \frac{\partial^{k} f}{\partial t^{k}} \otimes \rho\left(t^{k} \frac{\partial}{\partial t_{i}}\right) u .
$$

where $f, g \in \mathcal{A}_{(h)}$ and $u \in U$.

Considering left $\mathcal{A}$-action by multiplication and identifying the Lie algebra vector fields $\mathcal{V}$ with its natural embedding into $\operatorname{Der}\left(\mathcal{A}_{(h)}\right)$, we obtain $\mathcal{A} \mathcal{V}$-module structure on $\mathcal{A}_{(h)} \otimes U$.

Definition 34. An $\mathcal{A} \mathcal{V}$-submodule in $\mathcal{A}_{(h)} \otimes U$ which has finite rank over $\mathcal{A}$ is called a local gauge module. We say that an $\mathcal{A} \mathcal{V}$-module $M$ is a gauge module if it is isomorphic to a local gauge module for each chart $C(h)$ in our standard atlas.

In this chapter, we will focus on the case when $U$ is an irreducible finite-dimensional $\mathcal{L}_{+}$-module. It was shown in [3, Lemma 2] that such modules are just irreducible $\mathcal{L}_{0} \cong \mathfrak{g l}_{n^{-}}$ modules on which $\mathcal{L}_{s}$ with $s \geq 1$ act trivially. In this case the second axiom implies that the gauge fields $B_{i}$ are just functions in $\mathcal{A}_{(h)}$, and the third axiom of the gauge fields becomes $\frac{\partial B_{i}}{\partial t_{j}}=\frac{\partial B_{j}}{\partial t_{i}}$.

The action of $\operatorname{Der}\left(\mathcal{A}_{(h)}\right)$ on $\mathcal{A}_{(h)} \otimes U$ can be written as follows:

$$
\left(f \frac{\partial}{\partial t_{i}}\right) \cdot(g \otimes u)=f \frac{\partial g}{\partial t_{i}} \otimes u+f g B_{i} \otimes u+\sum_{p=1}^{N} g \frac{\partial f}{\partial t_{p}} \otimes \rho\left(E_{p i}\right) u .
$$

Given a gauge module $(M, \varphi)$ and a closed 1-form $\omega \in \Omega^{1}(X)$ on $X$, we can define a new gauge module structure $\varphi_{\omega}$ on the space $M$. Write $\omega$ in each chart $C(h)$ as $\omega=$ $P_{1} d t_{1}+\ldots+P_{N} d t_{N}$ with $P_{i} \in \mathcal{A}_{(h)}$. Since $\omega$ is closed, the functions $\left\{P_{i}\right\}$ will satisfy $\frac{\partial P_{i}}{\partial t_{j}}=\frac{\partial P_{j}}{\partial t_{i}}$. Then we define $\varphi_{\omega}$ in the chart $C(h)$ as

$$
\varphi_{\omega}\left(f \frac{\partial}{\partial t_{i}}\right) \cdot m=\varphi\left(f \frac{\partial}{\partial t_{i}}\right) \cdot m+f P_{i} m
$$

If the form $\omega$ is exact, $\omega=d(G)$ for some $G \in \mathcal{A}$, then module $\left(M, \varphi_{\omega}\right)$ may be formally interpreted as the space $e^{G} M$ with the "old" action $\varphi$ :

$$
\varphi\left(f \frac{\partial}{\partial t_{i}}\right) \cdot e^{G} m=e^{G} \varphi\left(f \frac{\partial}{\partial t_{i}}\right) \cdot m+e^{G} f \frac{\partial G}{\partial t_{j}} m .
$$

In the case when the first de Rham cohomology $H_{d R}^{1}(X)$ is non-zero, not every closed 1-form is exact, hence we can not always interpret $\varphi_{\omega}$ as a formal shift of the algebra of functions by a formal factor $e^{G}$.

Although we can construct new gauge modules by modifying the action with a help of a closed 1-form, an example of a family of rank 1 gauge modules for $X=\mathbb{S}^{2}$, given in [7], shows that we can not obtain all gauge modules in this way, starting from modules with zero gauge fields.

We recall the main theorem about gauge modules of [7]:

Theorem 35 ([7], Theorem 24). Let $X$ be a smooth irreducible affine algebraic variety and let $M$ be a gauge module which corresponds to a simple finite-dimensional $\mathfrak{g l}_{N}$-module $U$. Then $M$ is a simple $\mathcal{A} \mathcal{V}$-module. 
Our main goal of this chapter is to investigate the simplicity of these modules over $\mathcal{V}$. We are going to show that gauge modules remain irreducible as $\mathcal{V}$-modules, unless $U$ is an exterior power of the natural $N$-dimensional $\mathfrak{g l}_{N}$-module. This will be done by reconstructing the $\mathcal{A}$-action from the $\mathcal{V}$-action.

For the rest of the chapter, we will assume that $M$ is a gauge module which corresponds to a simple finite-dimensional $\mathfrak{g l}_{N}$-module $U$.

Recall from Proposition 16 that $Z\left(\mathcal{U}\left(\mathfrak{g l}_{N}\right)\right)=\mathbb{K}\left[\Omega_{1}, \ldots, \Omega_{N}\right]$, where

$$
\Omega_{k}=\sum_{i_{1}, \ldots, i_{k}=1}^{N} E_{i_{1} i_{2}} E_{i_{2} i_{3}} \cdots E_{i_{k} i_{1}} .
$$

Lemma 36. If a gauge module $M$ is reducible as a $\mathcal{V}$-module, then $\Omega_{1}$ acts on $U$ by a scalar from the set $\{0,1, \ldots, N\}$.

Proof. Let $M^{\prime}$ be a non-trivial $\mathcal{V}$-submodule in $M$. Let us fix one of the charts $C(h)$ of $X$ with its chart parameters. Let $f \in \mathcal{A}, i \in\{1,2, \ldots, N\}$, and $0 \leq r \leq 2$. Consider the composition of the actions of vector fields from $\mathcal{V}$ on $\mathcal{A}_{(h)} \otimes U$ :

$$
\left(t_{i}^{2-r} f h \frac{\partial}{\partial t_{i}}\right) \circ\left(t_{i}^{r} h \frac{\partial}{\partial t_{i}}\right)(g \otimes u)
$$

Expanding this expression using (3.1), we obtain a quadratic polynomial in $r$. Using the Vandermonde determinant we conclude that $M^{\prime}$ is invariant under the terms that correspond to each power of $r$.

The operator that corresponds to $-r^{2}$ is

$$
f h^{2}\left(E_{i i}^{2}-E_{i i}\right)
$$

and we conclude that $M^{\prime}$ is invariant under these operators.

Since $X$ is smooth, the set of functions $\left\{h_{j}\right\}$ determining the charts of $X$, has no common zeros on $X$. By the Corollary 24 of the Hilbert's Nullstellensatz, the ideal generated by $\left\{h_{j}^{2}\right\}$ coincides with $\mathcal{A}$. Hence, $M^{\prime}$ is invariant under $E_{i i}^{2}-E_{i i}$, and more generally $f\left(E_{i i}^{2}-E_{i i}\right)$ for any $f \in \mathcal{A}$.

Recall that $E_{i i}$ belongs to the standard Cartan of $\mathfrak{g l}_{N}$, so there is a basis of $U$ of eigenvectors for all $E_{i i}$. Consider the decomposition of $U$ into the joint eigenspaces for the family of commuting diagonalizable operators $E_{i i}^{2}-E_{i i}, i=1, \ldots N$.

For $m \in M^{\prime}$ consider its expansion in the joint eigenvectors for $E_{i i}^{2}-E_{i i}, m=\sum_{\alpha} m_{\alpha}$. Again we use the Vandermonde argument, so each component $m_{\alpha}$ is in $M^{\prime}$.

Let us assume that the zero eigenspace is trivial, it means that for each component $m_{\alpha}$ there exists at least one $i$ such that $E_{i i}^{2}-E_{i i}$ is acting by a non-zero scalar, hence for every function $f \in \mathcal{A}$ we get $f m_{\alpha} \in M^{\prime}$. This implies that $f m \in M^{\prime}$ and $M^{\prime}$ is in fact an $\mathcal{A} \mathcal{V}$-submodule in $M$. But Theorem 35 states that $M$ is irreducible as an $\mathcal{A} \mathcal{V}$-module. Since $M^{\prime}$ is a proper submodule, this is a contradiction with our assumption that the zero eigenspace is trivial. So we conclude that the zero eigenspace is, in fact, non-trivial. This means that each $E_{i i}$ is acting on this space as either 0 or 1 . But then $\Omega_{1}=\sum_{i=1}^{N} E_{i i}$ acts on $U$ by a scalar from $\{0,1, \ldots, N\}$.

We will use the notation $I_{N}=\{1,2, \ldots, N\}$ below. 
Proposition 37. Let $k \geq 2$. For an arbitrary $f \in \mathcal{A}$ a $\mathcal{V}$-submodule $M^{\prime}$ in $M$ is invariant under the action of

$$
f\left(\sum_{i \in I_{N}^{k}} \sum_{\sigma \in S_{k}} E_{i_{\sigma(1)} i_{1}} E_{i_{\sigma(2)} i_{2}} \ldots E_{i_{\sigma(k)} i_{k}}-\frac{(N+k-1) !}{N !} \Omega_{1}\right) .
$$

Proof. Fix $s$ to be a large enough element in $\mathbb{Z}_{+}^{N}$ (it should be large enough for the Vandermonde arguments we use below to work, $s_{j} \geq k^{3}$ for all $j$ will suffice). Let $c \in \mathbb{K}^{N}$ and $f \in \mathcal{A}$. Let $r \in \mathbb{Z}_{+}^{N}$ with $0 \leq r_{j} \leq k$ for all $1 \leq j \leq N$ and let $0 \leq n_{2}, \ldots, n_{k} \leq k$. Denote by $\left\{\epsilon_{1}, \ldots, \epsilon_{N}\right\}$ the standard basis of $\mathbb{Z}^{N}$. We shall be using multi-index notations, with $(t+c)^{s}=\left(t_{1}+c_{1}\right)^{s_{1}} \ldots\left(t_{N}+c_{N}\right)^{s_{n}}$.

Consider the action of

$$
\left((t+c)^{s+\sum_{l=1}^{k} \epsilon_{i_{l}}-\left(n_{2}+\ldots+n_{k}\right) r} f h \frac{\partial}{\partial x_{i_{1}}}\right) \circ\left((t+c)^{n_{2} r} h \frac{\partial}{\partial x_{i_{2}}}\right) \circ \cdots \circ\left((t+c)^{n_{k} r} h \frac{\partial}{\partial x_{i_{k}}}\right) .
$$

As in the Lemma 36 we can expand this expression into a polynomial in $n_{2}, \ldots, n_{k}$ by applying (3.1). By a Vandermonde argument, $M^{\prime}$ is invariant under the action of each term in this polynomial. The action of the term corresponding to the monomial $n_{2}^{2} n_{3} \ldots n_{k}$ is given by

$$
(t+c)^{s} f h^{k}\left(r_{i_{1}}-\sum_{q_{1}} r_{q_{1}} E_{q_{1} i_{1}}\right)\left(\sum_{q_{2}} r_{q_{2}} E_{q_{2} i_{2}}\right) \cdots\left(\sum_{q_{k}} r_{q_{k}} E_{q_{k} i_{k}}\right)
$$

Since $c$ is arbitrary, $M^{\prime}$ is also invariant under

$$
f h^{k}\left(r_{i_{1}}-\sum_{q_{1}} r_{q_{1}} E_{q_{1} i_{1}}\right)\left(\sum_{q_{2}} r_{q_{2}} E_{q_{2} i_{2}}\right) \ldots\left(\sum_{q_{k}} r_{q_{k}} E_{q_{k} i_{k}}\right)
$$

Consider a sequence $\iota=\left(i_{1}, \ldots, i_{k}\right) \in I_{N}^{k}$. The permutation group $S_{k}$ acts naturally on the set $I_{N}^{k}$. Let us denote by $\operatorname{Stab}(\iota)$ the stabilizer of $\iota$ in $S_{k}$. Set

$$
\omega_{\iota}=\frac{1}{|\operatorname{Stab}(\iota)|} \sum_{\sigma \in S_{k}} E_{i_{\sigma(1)} i_{1}} E_{i_{\sigma(2)} i_{2}} \ldots E_{i_{\sigma(k)} i_{k}} .
$$

For a sequence $\iota \in I_{N}^{k}$ denote by $\bar{i}$ the truncated sequence $\bar{i}=\left(i_{2}, \ldots, i_{k}\right) \in I_{N}^{k-1}$. Then the coefficient of $-r_{i_{1}} r_{i_{2}} \ldots r_{i_{k}}$ in (3.3) is $f h^{k}\left(\omega_{\iota}-\omega_{\bar{i}}\right)$ and $M^{\prime}$ is invariant under the action of this operator. By recursion on $k$ we conclude that $M^{\prime}$ is invariant under $f h^{k}\left(\omega_{\iota}-E_{i_{k} i_{k}}\right)$.

Multiplying these expressions by $|\operatorname{Stab}(\iota)|$ and taking a sum over all $\iota$, we get that $M^{\prime}$ is invariant under

$$
f h^{k}\left(\sum_{\iota \in I_{N}^{k}} \sum_{\sigma \in S_{k}} E_{i_{\sigma(1)} i_{1}} E_{i_{\sigma(2)} i_{2}} \ldots E_{i_{\sigma(k)} i_{k}}-\frac{1}{N} \sum_{\iota \in I_{N}^{k}}|\operatorname{Stab}(\iota)| \Omega_{1}\right) .
$$

Let $\left\{O_{j}\right\}$ be the set of orbits of $S_{k}$ in $I_{N}^{k}$. Each orbit can be thought of as a $k$ combination with repetitions from a set of $N$ elements. By standard combinatorics, the number of the orbits is $\left(\begin{array}{c}N+k-1 \\ k\end{array}\right)$. Fix a representative $\iota_{j}$ in each orbit $O_{j}$. 
Then we have:

$$
\sum_{\iota}|\operatorname{Stab}(\iota)|=\sum_{j}\left|O_{j}\right| \cdot\left|\operatorname{Stab}\left(\iota_{j}\right)\right|=\sum_{j}\left|S_{k}\right|=\left(\begin{array}{c}
N+k-1 \\
k
\end{array}\right) k !=\frac{(N+k-1) !}{(N-1) !}
$$

and $M^{\prime}$ is invariant under

$$
f h^{k}\left(\sum_{\iota \in I_{N}^{k}} \sum_{\sigma \in S_{k}} E_{i_{\sigma(1)} i_{1}} E_{i_{\sigma(2)} i_{2}} \ldots E_{i_{\sigma(k)} i_{k}}-\frac{(N+k-1) !}{N !} \Omega_{1}\right) .
$$

Since this is true for each chart of $X$, we can apply Hilbert's Nullstellensatz to the ideal generated by $\left\{h_{j}^{k}\right\}$ and drop $h^{k}$ in the above formula, obtaining the claim of the proposition.

\section{Lemma 38.}

$$
\widehat{\Omega}_{k}:=\sum_{i \in I_{N}^{k}} \sum_{\sigma \in S_{k}} E_{i_{\sigma(1)} i_{1}} E_{i_{\sigma(2)} i_{2}} \ldots E_{i_{\sigma(k)} i_{k}}
$$

belongs to $Z\left(\mathcal{U}\left(\mathfrak{g l}_{N}\right)\right)$.

Proof. Fix $a, b \in I_{N}$. We need to show that $\left[E_{a b}, \widehat{\Omega}_{k}\right]=0$ in $\mathcal{U}\left(\mathfrak{g l}_{N}\right)$. We can evaluate this commutator as follows:

$$
\begin{aligned}
& \sum_{\sigma \in S_{k}} \sum_{l \in I_{k}} \sum_{i \in I_{N}^{k}} \delta_{b i_{\sigma(l)}} E_{i_{\sigma(1)} i_{1}} \ldots E_{a i_{l}} \ldots E_{i_{\sigma(k)} i_{k}}-\delta_{a i_{l}} E_{i_{\sigma(1)} i_{1}} \ldots E_{i_{\sigma(l)} b} \ldots E_{i_{\sigma(k)} i_{k}} \\
= & \sum_{\sigma \in S_{k}} \sum_{l \in I_{k}} \sum_{\substack{i \in I_{N}^{k} \\
i_{\sigma(l)}=b}} E_{i_{\sigma(1)} i_{1}} \ldots E_{a i_{l}} \ldots E_{i_{\sigma(k)} i_{k}}-\sum_{\sigma \in S_{k}} \sum_{l \in I_{k}} \sum_{\substack{i \in I_{N}^{k} \\
i_{l}=a}} E_{i_{\sigma(1)} i_{1}} \ldots E_{i_{\sigma(l)} b} \ldots E_{i_{\sigma(k)} i_{k}} .
\end{aligned}
$$

Denote the product in the first sum as $\Gamma^{+}(\sigma, l, i)$ and the product in the second sum as $\Gamma^{-}(\sigma, l, i)$. The terms $\Gamma^{-}(\sigma, l, i)$ appear only for $i$ with $i_{l}=a$. For each such $i=$ $\left(i_{1}, \ldots, a, \ldots, i_{k}\right)$ set $\widetilde{i}=\left(i_{1}, \ldots, b, \ldots, i_{k}\right)$ with $\widetilde{i}_{l}=b$. Set $\widetilde{l}=\sigma^{-1}(l)$. Then we claim that for $i$ with $i_{l}=a$ we have $\Gamma^{-}(\sigma, l, i)=\Gamma^{+}(\sigma, \widetilde{l}, \widetilde{i})$. Note that $\Gamma^{+}(\sigma, \widetilde{l}, \widetilde{i})$ will appear in the summation since $\widetilde{i}_{\sigma(\widetilde{l})}=\widetilde{i}_{l}=b$. Indeed both products $\Gamma^{+}(\sigma, \widetilde{l}, \widetilde{i})$ and $\Gamma^{-}(\sigma, l, i)$ will have $E_{i_{\sigma(l)} b}$ as $l$-th factor and $E_{a \tilde{\tau}_{\bar{l}}}$ as $\tilde{l}$-th factor, and all other factors are the same as well.

Since we have a bijective correspondence between the terms $\Gamma^{+}$and $\Gamma^{-}$, all terms will cancel, showing that the Lie bracket is indeed zero.

Corollary 39. There exist at most $N+1$ simple finite-dimensional $\mathfrak{g l}_{N}$-modules $U$, for which simple gauge $\mathcal{A} \mathcal{V}$-modules become reducible when viewed as modules over $\mathcal{V}$.

Proof. Lemma 38 guarantees that $\widehat{\Omega}_{k}$ can be written as a polynomial in Casimirs $\Omega_{i}$, we set

$$
P_{k}\left(\Omega_{1}, \ldots, \Omega_{k}\right):=\widehat{\Omega}_{k}-\frac{(N+k-1) !}{N !} \Omega_{1} .
$$

Note that for $2 \leq k \leq N$, the Casimir $\Omega_{k}$ will occur in $P_{k}\left(\Omega_{1}, \ldots, \Omega_{k}\right)$ with a non-zero coefficient, so $P_{k}$ may be written as $P_{k}\left(\Omega_{1}, \ldots, \Omega_{k}\right)=c \Omega_{k}+Q_{k}\left(\Omega_{1}, \ldots, \Omega_{k-1}\right)$. Indeed, the expression for $\widehat{\Omega}_{k}$ contains the term $E_{12} E_{23} \ldots E_{k 1}$, while such a term can not come from the products of the Casimirs of lower orders. 
From Lemma 37 we know that $M^{\prime}$ is invariant under the action of $f P_{k}\left(\Omega_{1}, \ldots, \Omega_{k}\right)$. If any of these polynomials acts as a non-zero scalar on $U$ then we can reconstruct the $\mathcal{A}$ action and conclude that $M^{\prime}$ is an $\mathcal{A} \mathcal{V}$-submodule in $M$, which will contradict simplicity of $M$ as an $\mathcal{A} \mathcal{V}$-module.

If all polynomials $P_{k}\left(\Omega_{1}, \ldots, \Omega_{k}\right)$ for $2 \leq k \leq N$ act on $U$ as zero, each one will be fixing the value of one Casimir in terms of the values of the lower Casimirs, so if we fix the action of $\Omega_{1}$ and all the polynomials are acting by zero we will be fixing the central character of $U$. By the Harish-Chandra Isomorphism we have at most one finitedimensional simple $\mathfrak{g l}_{N}$-module for each central character, see e.g. [17, Chapter 1]. Thus we have at most $N+1$ simple $\mathfrak{g l}_{N}$-modules that give rise to reducible $\mathcal{V}$-modules, since by Lemma 36 we have $N+1$ possible values for the action of $\Omega_{1}$.

Remark 40. Note that the polynomials $P_{k}\left(\Omega_{1}, \ldots, \Omega_{k}\right)$ are determined by the $\mathfrak{g l}_{N}$-module $U$ and do not depend on neither the gauge fields $\left\{B_{i}\right\}$, nor on the variety $X$.

Example: $P_{2}\left(\Omega_{1}, \Omega_{2}\right)$ is given by $\Omega_{2}+\Omega_{1}^{2}-(N+1) \Omega_{1}$. For $N=2$, if we take $\Omega_{1} \in$ $\{0,1,2\}$ and obtain $\Omega_{2}$ by solving $P_{2}\left(\Omega_{1}, \Omega_{2}\right)=0$, we obtain the following three central characters:

\begin{tabular}{|l||l|l|l|}
\hline$\Omega_{1}$ & 0 & 1 & 2 \\
\hline$\Omega_{2}$ & 0 & 2 & 2 \\
\hline
\end{tabular}

which are the central characters of the modules of exterior powers of the natural module $\mathbb{K}^{2}$.

\subsection{De Rham complex}

Let $V$ be the natural $\mathfrak{g l}_{N}$-module. It has a basis $\left\{e_{1}, \ldots, e_{N}\right\}$ on which $\mathfrak{g l}_{N}$ acts by $E_{i j} \cdot e_{k}=\delta_{j k} e_{i}$. This action extends naturally to $\Lambda^{k} V$ in the standard way: for $x \in \mathfrak{g l}_{n}$ we have

$$
x \cdot\left(e_{i_{1}} \wedge \cdots \wedge e_{i_{k}}\right)=\sum_{j=1}^{k} e_{i_{1}} \wedge \cdots \wedge\left(x \cdot e_{i_{j}}\right) \wedge \cdots \wedge e_{i_{k}} .
$$

We also extend this definition to the trivial case and define $\Lambda^{0} V=\mathbb{K}$ to be 1-dimensional with all of $\mathfrak{g l}_{N}$ acting as zero. From Proposition 15 , each $\Lambda^{k} V$ is a simple $\mathfrak{g l}_{N^{-}}$-module on which the identity matrix acts by the scalar $k$.

Let $B=\left(B_{1}, \ldots, B_{N}\right)$ with $B_{i} \in \mathcal{A}_{(h)}$ such that $\frac{\partial}{\partial x_{i}}\left(B_{j}\right)=\frac{\partial}{\partial x_{j}}\left(B_{i}\right)$. For example we may pick $B=\nabla G$ for a fixed function $G \in \mathcal{A}$. Then for $1 \leq k \leq N$, we have an $\mathcal{A} \mathcal{V}$ module structure on $\mathcal{A}_{(h)} \otimes \Lambda^{k} V$ where a vector field $\sum f_{i} \frac{\partial}{\partial x_{i}}$ as embedded in $\operatorname{Der}\left(\mathcal{A}_{(h)}\right)$ acts via

$$
\left(\sum_{i} f_{i} \frac{\partial}{\partial x_{i}}\right) \cdot g \otimes v=\sum_{i}\left(f_{i} \frac{\partial g}{\partial x_{i}}+B_{i} f_{i} g\right) \otimes v+\sum_{i, p} \frac{\partial f_{i}}{\partial x_{p}} g \otimes E_{p i} v .
$$

Now we define maps $d_{k}: \mathcal{A}_{(h)} \otimes \Lambda^{k} V \rightarrow \mathcal{A}_{(h)} \otimes \Lambda^{k+1} V$ by

$$
d_{k}(g \otimes v)=\sum_{p=1}^{N}\left(\frac{\partial g}{\partial x_{p}}+B_{p} g\right) \otimes e_{p} \wedge v
$$


Proposition 41. The maps $d_{k}$ are morphisms of Lie algebra modules which satisfy $d_{k+1} \circ$ $d_{k}=0$. In other words,

$$
\mathcal{A}_{(h)} \otimes \Lambda^{0} V \stackrel{d_{0}}{\longrightarrow} \mathcal{A}_{(h)} \otimes \Lambda^{1} V \stackrel{d_{1}}{\longrightarrow} \cdots \stackrel{d_{N-1}}{\longrightarrow} \mathcal{A}_{(h)} \otimes \Lambda^{N} V
$$

is a chain complex in the category $\mathcal{V}$-Mod.

Proof. To prove that $d_{k}$ is a homomorphism of Lie algebras we will apply $d_{k} \circ f \partial / \partial x_{i}$ and $d_{k} \circ f \partial / \partial x_{i}$ to $g \otimes v$, we have:

$$
\begin{aligned}
& d_{k} \circ f \frac{\partial}{\partial x_{i}}(g \otimes v)=d_{k}\left(f \frac{\partial g}{\partial x_{i}}+B_{i} f g \otimes v+\sum_{p} \frac{\partial f}{\partial x_{p}} g \otimes E_{p i} v\right) \\
= & \sum_{q}\left(\frac{\partial}{\partial x_{q}}\left(f \frac{\partial g}{\partial x_{i}}+B_{i} f g\right)+B_{q} f \frac{\partial g}{\partial x_{i}}+B_{q} B_{i} f g\right) \otimes e_{q} \wedge v \\
+ & \sum_{p, q}\left(\frac{\partial}{\partial x_{q}}\left(\frac{\partial f}{\partial x_{p}} g\right)+B_{q} \frac{\partial f}{\partial x_{p}} g\right) \otimes e_{q} \wedge E_{p i} v
\end{aligned}
$$

and

$$
\begin{aligned}
& f \frac{\partial}{\partial x_{i}} \circ d_{k}(g \otimes v)=f \frac{\partial}{\partial x_{i}}\left(\sum_{q}\left(\frac{\partial g}{\partial x_{q}}+B_{q} g\right) \otimes e_{q} \wedge v\right) \\
= & \sum_{q}\left(f \frac{\partial}{\partial x_{i}}\left(\frac{\partial g}{\partial x_{q}}+B_{q} g\right)+B_{i} f \frac{\partial g}{\partial x_{q}}+B_{q} B_{i} f g\right) \otimes e_{q} \wedge v \\
+ & \sum_{p, q} \frac{\partial f}{\partial x_{p}}\left(\frac{\partial g}{\partial x_{q}}+B_{q} g\right) \otimes E_{p i}\left(e_{q} \wedge v\right)
\end{aligned}
$$

Observe that $E_{p i}\left(e_{q} \wedge v\right)=E_{p i} e_{q} \wedge v+e_{q} \wedge E_{p i} v$ and the first term is not zero only when $q=i$. Subtracting the second from the first equation, we have:

$$
\begin{aligned}
& \left(d_{k} \circ f \frac{\partial}{\partial x_{i}}-f \frac{\partial}{\partial x_{i}} \circ d_{k}\right)(g \otimes v) \\
= & \sum_{q}\left(\frac{\partial f}{\partial x_{q}} \frac{\partial g}{\partial x_{i}}+\frac{\partial}{\partial x_{q}}\left(B_{i} f g\right)+B_{q} f \frac{\partial g}{\partial x_{i}}-f \frac{\partial}{\partial x_{i}}\left(B_{q} g\right)-B_{i} f \frac{\partial g}{\partial x_{q}}\right) \otimes e_{q} \wedge v \\
+ & \sum_{p, q} \frac{\partial^{2} f}{\partial x_{q} \partial x_{p}} g \otimes e_{q} \wedge E_{p i} v-\sum_{p} \frac{\partial f}{\partial x_{p}}\left(\frac{\partial g}{\partial x_{i}}+B_{i} g\right) \otimes e_{p} \wedge v \\
= & \sum_{p, q} \frac{\partial^{2} f}{\partial x_{q} \partial x_{p}} g \otimes e_{q} \wedge E_{p i} v
\end{aligned}
$$

We claim that this last term is equal to zero. We will prove in the case that $v$ is basis vector and the general proof follows by linearity. Suppose $v=e_{j_{1}} \wedge \ldots e_{j_{k}}$, if none $j_{l}$ is equal to $i$ then $E_{p i}$ is acting as zero and we have our claim. Without loss of generality, let us suppose that $j_{1}=i$. Now, the previous equation becomes

$$
\begin{aligned}
& \sum_{p, q} \frac{\partial^{2} f}{\partial x_{q} \partial x_{p}} g \otimes e_{q} \wedge E_{p i} e_{i} \wedge e_{j_{2}} \wedge \ldots \wedge e_{j_{k}} \\
= & \sum_{p<q} \frac{\partial^{2} f}{\partial x_{q} \partial x_{p}} g \otimes e_{q} \wedge e_{p} \wedge e_{j_{2}} \wedge \ldots \wedge e_{j_{k}}+\sum_{p>q} \frac{\partial^{2} f}{\partial x_{q} \partial x_{p}} g \otimes e_{q} \wedge e_{p} \wedge e_{j_{2}} \wedge \ldots \wedge e_{j_{k}} \\
= & \sum_{p<q} \frac{\partial^{2} f}{\partial x_{q} \partial x_{p}} g \otimes e_{q} \wedge e_{p} \wedge e_{j_{2}} \wedge \ldots \wedge e_{j_{k}}-\sum_{p<q} \frac{\partial^{2} f}{\partial x_{p} \partial x_{q}} g \otimes e_{q} \wedge e_{p} \wedge e_{j_{2}} \wedge \ldots \wedge e_{j_{k}}
\end{aligned}
$$

In the last step we switched indices $p$ and $q$ in the second summation. Then we used $e_{p} \wedge e_{q}=-e_{q} \wedge e_{p}$. This proves our claim and we have that the $d_{k}$ are, indeed, $\mathcal{V}$-module morphisms. Note however that the maps $d_{k}$ are not $\mathcal{A}$-module morphisms.

To prove that $d_{k+1} \circ d_{k}=0$ let us fix the notation

$$
\Upsilon(p, q)=\frac{\partial^{2} g}{\partial x_{q} \partial x_{p}}+\frac{\partial B_{p}}{\partial x_{q}} g+B_{p} \frac{\partial g}{\partial x_{q}}+B_{q} \frac{\partial g}{\partial x_{p}}+B_{q} B_{p} g
$$


Applying $d_{k+1} \circ d_{k}$ to $(g \otimes v)$, we get:

$$
\begin{aligned}
& d_{k+1} \circ d_{k}(g \otimes v)=d_{k+1}\left(\sum_{p}\left(\frac{\partial g}{\partial x_{p}}+B_{p} g\right) \otimes e_{p} \wedge v\right) \\
= & \sum_{p, q} \frac{\partial}{\partial x_{q}}\left(\frac{\partial g}{\partial x_{p}}+B_{p} g\right)+B_{q} \frac{\partial g}{\partial x_{p}}+B_{q} B_{p} g \\
= & \sum_{p, q} \Upsilon(p, q) \otimes e_{q} \wedge e_{p} \wedge v \\
= & \sum_{p<q} \Upsilon(p, q) \otimes e_{q} \wedge e_{p} \wedge v+\sum_{p>q} \Upsilon(p, q) \otimes e_{q} \wedge e_{p} \wedge v \\
= & \sum_{p<q} \Upsilon(p, q) \otimes e_{q} \wedge e_{p} \wedge v-\sum_{p<q} \Upsilon(q, p) \otimes e_{q} \wedge e_{p} \wedge v \\
= & \sum_{p<q}(\Upsilon(p, q)-\Upsilon(q, p)) \otimes e_{q} \wedge e_{p} \wedge v .
\end{aligned}
$$

Since $\frac{\partial}{\partial x_{p}}\left(B_{q}\right)=\frac{\partial}{\partial x_{q}}\left(B_{p}\right)$, we have that $\Upsilon(p, q)=\Upsilon(q, p)$. So the last equation equals to zero and this concludes the proof.

We can now state the main theorem about gauge modules.

Theorem 42. If $U$ is a simple $\mathfrak{g l}_{N}$-module that is not an exterior power of the natural module, then any gauge module $M \subset \mathcal{A}_{(h)} \otimes U$ is simple as a $\mathcal{V}$-module.

Proof. By Lemma 36 a gauge module $M \subset \mathcal{A}_{(h)} \otimes U$ is simple in $\mathcal{V}$-Mod if $\Omega_{1}=\sum_{i=1}^{N} E_{i i}$ does not act on $U$ as a scalar belonging from the set $\{0, \ldots, N\}$. Moreover, by the discussion after Lemma 38, there exist at most $N+1$ exceptional $\mathfrak{g l}_{N}$-modules which may correspond to $\mathcal{V}$-reducible gauge modules.

It therefore only remains to show that there exist $\mathcal{V}$-reducible gauge modules precisely when $U=\Lambda^{k} V$ for $0 \leq k \leq N$. The theorem of Rao already guarantees this for the torus, but now we present another construction using the affine space: take $X=\mathbb{A}^{N}$, and $h=1$. Then the chart $C(h)$ covers $X$, the standard variables are chart parameters, and $\mathcal{A}_{(h)}=\mathcal{A}=\mathbb{K}\left[x_{1}, \ldots, x_{N}\right]$. Picking all $B_{i}=0$, we have a gauge module structure on each $\mathcal{A} \otimes \Lambda^{k} V$ for $0 \leq k \leq N$ where a vector field acts by

$$
\left(\sum_{i=1}^{N} f_{i} \frac{\partial}{\partial x_{i}}\right) \cdot g \otimes v=\sum_{i=1}^{N} f_{i} \frac{\partial g}{\partial x_{i}} \otimes v+\sum_{i, p=1}^{N} \frac{\partial f_{i}}{\partial x_{p}} g \otimes E_{p i} v .
$$

Since the maps $d_{k}$ from Proposition 41 are $\mathcal{V}$-module homomorphisms, the kernel of $d_{k}$ is a submodule for $\mathcal{A} \otimes \Lambda^{k} V$ for $0 \leq k<N$. Since $d_{k}\left(1 \otimes e_{1} \wedge \cdots \wedge e_{k}\right)=0$ but $d_{k}\left(x_{1} \otimes e_{2} \wedge \cdots \wedge e_{k+1}\right)=e_{1} \wedge \cdots \wedge e_{k+1} \neq 0$, we see that Ker $d_{k}$ is in fact a proper submodule, so $\mathcal{A} \otimes \Lambda^{k} V$ is a reducible $\mathcal{V}$-module for $0 \leq k<N$.

Now only the case $k=N$ remains and we need to find an example of a reducible gauge module structure on $\mathcal{A} \otimes \Lambda^{N} V$. In the above example, this module is actually simple, so instead we pick $B_{i}=-2 x_{i}$. We claim that

$$
\operatorname{Im} d_{N-1}=\left\{\sum_{i=1}^{N}\left(\frac{\partial f_{i}}{\partial x_{i}}-2 x_{i} f_{i}\right) \otimes e_{1} \wedge \cdots \wedge e_{N} \mid f_{1}, \ldots, f_{N} \in \mathbb{K}\left[x_{1}, \ldots, x_{N}\right]\right\}
$$

is a proper submodule in $\mathcal{A} \otimes \Lambda^{N} V$. Indeed, this submodule is non-zero and does not contain the element $1 \otimes e_{1} \wedge \cdots \wedge e_{N}$. To see this, we interpret de Rham complex with these gauge fields as

$$
e^{G} \Omega^{0} \stackrel{d_{0}}{\longrightarrow} \ldots \stackrel{d_{N-2}}{\longrightarrow} e^{G} \Omega^{N-1} \stackrel{d_{N-1}}{\longrightarrow} e^{G} \Omega^{N}
$$


where $G=-x_{1}^{2}-\ldots-x_{N}^{2}$. Let us give an analytic proof that $e^{-x_{1}^{2}-\ldots-x_{N}^{2}} d x_{1} \wedge \ldots \wedge d x_{N} \notin$ Im $d_{N-1}$ under assumption that $\mathbb{R} \subset \mathbb{K}$. Let $\omega \in e^{G} \Omega^{N-1}$. Applying Stokes' theorem, we see that

$$
\int_{\mathbb{R}^{N}} d \omega=0
$$

however

$$
\int_{\mathbb{R}^{N}} e^{-x_{1}^{2}-\ldots-x_{N}^{2}} d x_{1} \wedge \ldots \wedge d x_{N}=\pi^{N / 2}
$$

Hence, $e^{-x_{1}^{2}-\ldots-x_{N}^{2}} d x_{1} \wedge \ldots \wedge d x_{N} \notin \operatorname{Im} d_{N-1}$.

\subsection{Gauge modules on $\mathbb{S}^{1}$ and irreducible modules for $\mathfrak{s l}_{2}$}

In Theorem 42 we proved that gauge modules corresponding to non-exceptional irreducible $\mathfrak{g l}_{N}$ modules $U$, are simple $\mathcal{V}$-modules. In this section, we are going to show that the irreducibility of $U$ is not a necessary condition for simplicity of a gauge $\mathcal{V}$-module.

In this section we fix $X=\mathbb{S}^{1}$ with equation $x^{2}+y^{2}=1$. Setting $t=x+\sqrt{-1} y$, $s=x-\sqrt{-1} y$, we rewrite the equation of the circle as $t s=1$. The jacobian matrix is $(s t)$, and we see that the chart $s \neq 0$ covers the whole circle, which allows us to work with a single chart with the chart parameter $t$. Since $s$ is invertible in $\mathcal{A}$, the localized algebra $\mathcal{A}_{(s)}$ coincides with $\mathcal{A}=\mathbb{K}\left[t, t^{-1}\right]$.

For $\alpha \in K$, let us consider the following gauge module for the Lie algebra of vector fields on a circle, $W_{1}=\operatorname{Der}(\mathbb{K})\left[t, t^{-1}\right]$. Take $U_{\alpha}=\mathbb{K}^{2}$ with a basis $\{v, u\}$ and the identity matrix in $\mathfrak{g l}_{1}$ acting on $U_{\alpha}$ as multiplication by $\alpha$. Obviously $U_{\alpha}$ splits as a direct sum of two isomorphic 1-dimensional $\mathfrak{g l}_{1}$-modules.

Since our variety is 1 -dimensional and $\mathfrak{g l}_{1}$ acts on $U_{\alpha}$ by scalar matrices, any $2 \times 2$ matrix $B$ with entries in $\mathcal{A}$ will define a gauge field on $N(\alpha)=\mathcal{A} \otimes U_{\alpha}$. For our example we set

$$
B=\left(\begin{array}{ll}
0 & t \\
1 & 0
\end{array}\right)
$$

Setting a basis $v_{k}=t^{k} \otimes v, u_{k}=t^{k} \otimes u$, the action of $W_{1}$ on $N(\alpha)$ can be written as

$$
\begin{aligned}
& e_{n} v_{k}=(k+\alpha n) v_{n+k}+u_{n+k}, \\
& e_{n} u_{k}=(k+\alpha n) u_{n+k}+v_{n+k+1},
\end{aligned}
$$

where $e_{n}=t^{n+1} \frac{\partial}{\partial t}, n, k \in \mathbb{Z}$.

The span of $\left\{e_{1}, e_{0}, e_{-1}\right\}$ forms a subalgebra in $W_{1}$, which is isomorphic to $\mathfrak{s l}_{2}$. We are going to show that $N(0)$ is a simple $W_{1}$-module by studying its structure as a module over $\mathfrak{s l}_{2}$.

It is easy to check that the Casimir element $C=e_{0}^{2}+e_{0}-e_{-1} e_{1}$ acts on $N(\alpha)$ as multiplication by $\gamma=\alpha(\alpha-1)$. We can view $N(\alpha)$ as a module over the quotient algebra $U(\gamma)=U\left(\mathfrak{s l}_{2}\right) /\langle C-\gamma\rangle$.

R. Block [9] classified irreducible $\mathfrak{s l}_{2}$-modules by describing maximal left ideals in the algebras $U(\gamma)$. Simple modules are then presented as quotients by these left ideals. For general non-weight simple modules, explicit realizations in terms of action on a basis are not known. A slightly different approach to Block's classification is given by Bavula in 
[2]. We will rely on the results of [2] in order to understand the structure of $N(\alpha)$ as a module over $\mathfrak{s l}_{2}$.

Theorem 43. (a) (i) The vector $v_{0} \in N(0)$ is annihilated by $s=e_{-1} e_{0}-1 \in U(0)$.

(ii) An $\mathfrak{s l}_{2}$-submodule $M \subset N(0)$ generated by $v_{0}$ is a simple $\mathfrak{s l}_{2}$-module and

$$
M \cong U(0) / U(0) s .
$$

(iii) The set $\left\{v_{0}, e_{0}^{n} v_{0}, e_{-1}^{n} v_{0} \mid n \geq 1\right\}$ is a basis of $M$.

(iv) The quotient $N(0) / M$ is a simple highest weight $\mathfrak{s l}_{2}$-module with the highest weight -1 .

(v) $N(0)$ is irreducible as a $W_{1}$-module.

(b) Let $\alpha \notin \frac{1}{2} \mathbb{Z}, \gamma=\alpha(\alpha-1)$.

(i) The vector $v_{0} \in N(\alpha)$ is annihilated by $p=e_{1}-e_{0}^{2}\left(e_{0}+1-\alpha\right)$ and $q=e_{-1} e_{0}^{2}-\left(e_{0}+1-\alpha\right)$.

(ii) $N(\alpha)$ is a simple $\mathfrak{s l}_{2}$-module and

$$
N(\alpha) \cong U(\gamma) /(U(\gamma) p+U(\gamma) q)
$$

(iii) $N(\alpha)$ is irreducible as $W_{1}$-module.

Proof. Let us prove part (a) of the theorem. It is straightforward to check that $\left(e_{-1} e_{0}-\right.$ 1) $v_{0}=0$ in $N(0)$. By Corollary 3.9(b) in [2], the module $U(0) / U(0) s$ is a simple $\mathfrak{s l}_{2^{-}}$ module. Since the Casimir element $C$ acts trivially on $N(0)$, we have a homomorphism of $\mathfrak{s l}_{2}$-modules $\varphi: U(0) \rightarrow N(0)$, given by $\varphi(x)=x v_{0}$. Since $s$ annihilates $v_{0}$, the left ideal $U(0) s$ is in the kernel of $\varphi$ and we get a homomorphism

$$
\bar{\varphi}: U(0) / U(0) s \rightarrow N(0) .
$$

Since $U(0) / U(0) s$ is simple and $\bar{\varphi}(1)=v_{0}$, we conclude that $\bar{\varphi}$ is injective and its image is the $\mathfrak{s l}_{2}$-submodule $M \subset N(0)$, generated by $v_{0}$.

Let us introduce a linear order on the basis elements of $N(0)$ :

$$
\ldots<u_{-1}<v_{0}<u_{0}<v_{1}<u_{1}<v_{2}<\ldots
$$

This order defines the highest term and the lowest term for any non-zero element in $N(0)$. We also set $N_{+}=\operatorname{Span}\left\{v_{k}, u_{k} \mid k \geq 0\right\}$.

Consider the sequence $v_{0}, e_{0} v_{0}, e_{0}^{2} v_{0}, e_{0}^{3} v_{0}, \ldots$. All of these vectors are in $N_{+}$and their leading terms are $v_{0}, u_{0}, v_{1}, u_{1}, \ldots$. Hence these vectors span $N_{+}$and $N_{+} \subset M \subset N(0)$. Since $N_{+}$is invariant under the action of the Borel subalgebra spanned by $\left\{e_{0}, e_{1}\right\}$, we conclude that $M=\mathbb{K}\left[e_{-1}\right] N_{+}$. Moreover $e_{-1} v_{k}, e_{-1} u_{m} \in N_{+}$for $k \geq 1$ and $m \geq 0$. Hence

$$
M=N_{+}+\mathbb{K}\left[e_{-1}\right] v_{0} .
$$

The lowest terms of the vectors $e_{-1} v_{0}, e_{-1}^{2} v_{0}, e_{-1}^{3} v_{0}, \ldots$ are non-zero multiples of $u_{-1}, u_{-2}$, $u_{-3}, \ldots$ Hence these vectors are linearly independent and we conclude that $\left\{v_{0}, e_{0}^{n} v_{0}, e_{-1}^{n} v_{0}\right.$ $\mid n \geq 1\}$ is a basis of $M$. The images of the vectors $v_{-1}, v_{-2}, v_{-3}, \ldots$ form a basis of the quotient space $N(0) / M$. It is easy to see that

$$
\begin{array}{ll}
e_{0} v_{-1}=-v_{-1} & \bmod M, \\
e_{1} v_{-1}=0 & \bmod M,
\end{array}
$$


and that the image of $v_{-1}$ generates $N(0) / M$ as an $\mathfrak{s l}_{2}$-module. Since the Verma module for $\mathfrak{s l}_{2}$ with the highest weight -1 is simple, we conclude that $N(0) / M$ is isomorphic to it.

Finally, let us show that $N(0)$ is simple as a $W_{1}$-module. We note that $e_{1}$ acts on $N(0)$ injectively and every non-zero element of $N(0)$ is moved into $N_{+} \subset M$ by a high enough power of $e_{1}$. Hence every non-zero $\mathfrak{s l}_{2}$ submodule in $N(0)$ contains $M$. Since $N(0) / M$ is simple, we conclude that $M$ is the only proper $\mathfrak{s l}_{2}$-submodule in $N(0)$. It is easy to see that $M$ is not closed under the action of $W_{1}$, hence $N(0)$ is a simple $W_{1}$-module.

Part (b) of the theorem may be proved using Corollary 3.5 in [2]. We omit this proof. 


\section{Chapter 4}

\section{Rudakov Modules}

In [29], Rudakov was interested in reducing the problem of classifying irreducible representation of infinite-dimensional Lie algebra to the problem of classifying irreducible representations of finite-dimensional Lie algebras. To do so, Rudakov studied the Cartan type algebras, which admit a filtration. For example, let us recall the filtration of $\mathbf{W}_{N}$ for the affine space. For a monomial $Q \subset \mathbb{K}\left[x_{1}, \ldots, x_{N}\right]$ of degree $d$ we define the degree of $Q \frac{\partial}{\partial x_{i}}$ to be $d-1$ and we define $\mathcal{L}(j)$ as a subspace of elements of degree less or equal to $j$. We have the filtration:

$$
\mathcal{L}=\mathcal{L}(-1) \supset \mathcal{L}(0)=\mathcal{L}_{+} \supset \mathcal{L}(1) \supset \mathcal{L}(2) \supset \ldots
$$

Recall that $\mathcal{L}_{+} / \mathcal{L}(1)=\mathcal{L}_{0}$ is isomorphic to $\mathfrak{g l}_{N}$. Given a $\mathbf{W}_{N}$-module $M$, Rudakov defined the height of $M$ to be the minimum $p$ such that $\mathcal{L}_{p} m=0$ for some non-zero $m \in M$. His theorem [29][Corollary 6.3] states that modules for the Lie algebras of Cartan type of height greater than one are isomorphic to

$$
\mathcal{U}(\mathcal{L}) \otimes_{\mathcal{U}\left(\mathcal{L}_{0}\right)} U
$$

here $U$ is an irreducible $\mathfrak{g l}_{N}$-modules.

For $\mathbf{W}_{N}$, Rudakov's theorem was claiming that irreducible modules where induced from a $\mathfrak{g l}_{N}$-module or of height 1 . The second part of his paper is dedicated to the study of induced modules of height one coming from irreducible $\mathfrak{g l}_{N}$-modules. This is the main theorem about modules of height 1 :

Theorem 44 ([29], Theorem 13.7). If $U$ is an irreducible $\mathfrak{g l}_{N}$-module which coincides with none of the exceptional modules $\left(\Lambda^{0}\left(\mathbb{K}^{N}\right)\right)^{*}, \ldots,\left(\Lambda^{N}\left(\mathbb{K}^{N}\right)\right)^{*}$ then the $\mathcal{L}$-module $\mathcal{U}(\mathcal{L}) \otimes \mathcal{U}\left(\mathcal{L}_{+}\right)$ $U$ is irreducible. Each module $\mathcal{U}(\mathcal{L}) \otimes_{\mathcal{U}\left(\mathcal{L}_{+}\right)}\left(\Lambda^{k}\left(\mathbb{K}^{N}\right)\right)^{*}$ contains a unique submodule ${ }^{1}$, which is generated by all of the singular vectors.

Here $\left(\Lambda^{k}\left(\mathbb{K}^{N}\right)\right)^{*}$ is the dual representation of the $k$-th exterior power of the natural module and a singular vector is a vector that is annihilated by $\mathcal{L}_{1}$ and does not belong to the top $U$. The irreducibility of an induced module is deeply linked to the existence of singular vectors.

\footnotetext{
${ }^{1}$ Rudakov calls such modules irregular.
} 


\subsection{Rudakov modules for arbitrary varieties}

In [7], Billig, Futorny, and Nilsson generalize the construction of Rudakov for any variety. In order to obtain an $\mathcal{A} \mathcal{V}$-module they also defined an $\mathcal{A}$-action on the module. Let us present their construction.

Let $X$ be a smooth irreducible affine variety, $p$ a point of $X, \mathcal{V}$ the Lie algebra of vector fields on $X$ and $\mathcal{A}$ the algebra of functions on $X$. Let $U$ be a simple finite-dimensional $\mathcal{L}_{+^{-}}$ module. By [3, Lemma 2], we have that $\mathcal{L}(1) U=(0)$, hence $U$ is an $\mathcal{L}_{+} / \mathcal{L}(1)$-module. The isomorphism of Lemma 29 (c) defines a $\mathcal{V}_{+}$-module structure on $U$ such that $\mathcal{V}(1) U=(0)$. We also define an $\mathcal{A}$-action on $U$ by evaluation: $f \cdot u:=f(p) u$ for $f \in \mathcal{A}$ and $u \in U$.

The Rudakov module $R_{p}(U)$ is defined as an induced module

$$
R_{p}(U):=\mathcal{A} \# \mathcal{U}(\mathcal{V}) \otimes_{\mathcal{A} \# \mathcal{U}\left(\mathcal{V}_{+}\right)} U .
$$

Theorem $45([7])$. Let $U$ be a finite-dimensional simple $\mathcal{V}_{+} / \mathcal{V}(1) \simeq \mathfrak{g l}_{N}(\mathbb{K})$-module, and let $p$ be a non-singular point of $X$. Then the corresponding Rudakov module $R_{p}(U)$ is a simple $A \mathcal{V}$-module.

Our goal is to investigate whether Rudakov modules remain irreducible as $\mathcal{V}$-modules. We define a chain of subspaces in the Rudakov module by $R_{0}(U):=1 \otimes U$ and $R_{i+1}(U):=$ $R_{i}(U)+\mathcal{V} \cdot R_{i}(U)$. We also let $R_{i}(U):=(0)$ for $i<0$. This gives a filtration

$$
R_{0}(U) \subset R_{1}(U) \subset R_{2}(U) \subset \ldots
$$

with $\cup_{i=0}^{\infty} R_{i}(U)$ equal to the whole module.

Lemma 46 ([7], Lemma 9). We have

(a) $\mathfrak{m}_{p} R_{l}(U) \subset R_{l-1}(U)$.

(b) $\mathcal{V}(j) R_{l}(U) \subset R_{l-j}(U)$ for all $j$.

Fix a non-zero vector $v \in R_{p}(U)$ and let $l$ be the smallest integer such that $v \in R_{l}(U)$. Consider the following elements of $\mathcal{V}$, given by Lemma 29 (b):

$$
\eta_{i}=\frac{\partial}{\partial t_{i}}+\text { terms of degree } \geq l .
$$

Then $\left\{\eta_{1}, \ldots, \eta_{N}\right\}$ is a basis of the space $\mathcal{V} / \mathcal{V}_{+}$. Note that for any $w \in R_{l-2}(U)$ we have $\eta_{i} \eta_{j} w=\eta_{j} \eta_{i} w$ since $\left[\eta_{i}, \eta_{j}\right] w \in \mathcal{V}(l-1) R_{l-2}(U) \subset R_{-1}(U)=(0)$. Using the PBW theorem and this commutative relation, we may write

$$
v=\sum_{i=1}^{\operatorname{dim} U} P_{i}\left(\eta_{1}, \ldots, \eta_{N}\right) \otimes u_{i},
$$

where $P_{i}\left(\eta_{1}, \ldots, \eta_{N}\right)$ are polynomials of degree $\leq l$, and $\left\{u_{i}\right\}$ is a basis of $U$.

Throughout this chapter we will be using a slightly different multi-index notation. Let $\alpha \in \mathbb{Z}^{s}$, we will write $\eta^{\alpha}=\eta_{1}^{\alpha_{1}} \ldots \eta_{N}^{\alpha_{N}}$ if all $\alpha_{i} \geq 0$, which is the usual multi-index notation, and set $\eta^{\alpha}=0$ if any $\alpha_{i}$ is strictly negative. We also set $|\alpha|=\alpha_{1}+\ldots+\alpha_{N}$.

Using this notation $v$ can also be written as

$$
v=\sum_{|\alpha| \leq k} \eta^{\alpha} \otimes u_{\alpha}
$$


with $u_{\alpha} \in U$.

The space $\mathcal{U}(\mathcal{V} / \mathcal{V}(l))$ is isomorphic to the space of polynomial of degree less than $l$ in the variables $\left\{\eta_{1}, \ldots, \eta_{N}\right\}$. The next lemma will provide some useful identities in this space.

Lemma 47. In $\mathcal{U}(\mathcal{V} / \mathcal{V}(l))$ the following equation holds

$$
\left(t_{i}^{2} \eta_{j}\right) \eta^{\alpha}=\eta^{\alpha}\left(t_{i}^{2} \eta_{j}\right)+\alpha_{i}\left(\alpha_{i}-1\right) \eta^{\alpha-2 \epsilon_{i}+\epsilon_{j}}-2 \alpha_{i} \eta^{\alpha-\epsilon_{i}}\left(t_{i} \eta_{j}\right)
$$

and, for $i \neq j$,

$$
\left(t_{i} t_{j} \eta_{j}\right) \eta^{\alpha}=\eta^{\alpha}\left(t_{i} t_{j} \eta_{j}\right)+\alpha_{i} \alpha_{j} \eta^{\alpha-\epsilon_{i}}-\alpha_{j} \eta^{\alpha-\epsilon_{j}}\left(t_{i} \eta_{j}\right)-\alpha_{i} \eta^{\alpha-\epsilon_{i}}\left(t_{j} \eta_{j}\right)
$$

Proof. First we will prove the commutator relation for degree 0 and then we will prove the claim of the lemma which is the commutator relations for elements of degree 1.

We have, if $\alpha_{i} \neq 0$,

$$
\left(t_{i} \eta_{j}\right) \eta^{\alpha}=\eta_{i}\left(t_{i} \eta_{j}\right) \eta^{\alpha-\epsilon_{i}}-\eta^{\alpha-\epsilon_{i}+\epsilon_{j}}=\ldots=\eta^{\alpha}\left(t_{i} \eta_{j}\right)-\alpha_{i} \eta^{\alpha-\epsilon_{i}+\epsilon_{j}}
$$

If $\alpha_{i}=0$, we have $\left(t_{i} \eta_{j}\right) \eta^{\alpha}=\eta^{\alpha}\left(t_{i} \eta_{j}\right)$. Recall that $\eta^{\alpha}=0$ in the case any $\alpha_{i}$ is less than 0 , so in both cases:

$$
\left(t_{i} \eta_{j}\right) \eta^{\alpha}=\eta^{\alpha}\left(t_{i} \eta_{j}\right)-\alpha_{i} \eta^{\alpha-\epsilon_{i}+\epsilon_{j}}
$$

For the degree one we will split the computation in two cases, the first is commuting $t_{i}^{2} \eta_{j}$ with $\eta^{\alpha}$, where $i$ can be equal to $j$. The second case is with $t_{i} t_{j} \eta_{j}$ where $i \neq j$.

Suppose $\alpha_{i} \neq 0$ :

$$
\begin{aligned}
& \left(t_{i}^{2} \eta_{j}\right) \eta^{\alpha}=\eta_{i}\left(t_{i}^{2} \eta_{j}\right) \eta^{\alpha-\epsilon_{i}}-2\left(t_{i} \eta_{j}\right) \eta^{\alpha-\epsilon_{i}} \\
= & \eta_{i}\left(t_{i}^{2} \eta_{j}\right) \eta^{\alpha-\epsilon_{i}}-2 \eta^{\alpha-\epsilon_{i}}\left(t_{i} \eta_{j}\right)+2\left(\alpha_{i}-1\right) \eta^{\alpha-2 \epsilon_{i}+\epsilon_{j}} \\
= & \eta^{\alpha}\left(t_{i}^{2} \eta_{j}\right)-2 \alpha_{i} \eta^{\alpha-\epsilon_{i}}\left(t_{i} \eta_{i}\right)+2\left(\left(\alpha_{i}-1\right)+\left(\alpha_{i}-2\right)+\ldots+1\right) \eta^{\alpha-2 \epsilon_{i}+\epsilon_{j}} \\
= & \eta^{\alpha}\left(t_{i}^{2} \eta_{j}\right)-2 \alpha_{i} \eta^{\alpha-\epsilon_{i}}\left(t_{i} \eta_{j}\right)+\alpha_{i}\left(\alpha_{i}-1\right) \eta^{\alpha-2 \epsilon_{i}+\epsilon_{j}}
\end{aligned}
$$

if $\alpha_{i}=0$ we have $\left(t_{i}^{2} \eta_{j}\right) \eta^{\alpha}=\eta^{\alpha}\left(t_{i}^{2} \eta_{j}\right)$. So, in both cases we have

$$
\left(t_{i}^{2} \eta_{j}\right) \eta^{\alpha}=\eta^{\alpha}\left(t_{i}^{2} \eta_{j}\right)+\alpha_{i}\left(\alpha_{i}-1\right) \eta^{\alpha-2 \epsilon_{i}+\epsilon_{j}}-2 \alpha_{i} \eta^{\alpha-\epsilon_{i}}\left(t_{i} \eta_{j}\right)
$$

Similarly, we have for $t_{i} t_{j} \eta_{j}$ with $i \neq j$ and $\alpha_{i} \neq 0 \neq \alpha_{j}$ :

$$
\begin{aligned}
& \left(t_{i} t_{j} \eta_{j}\right) \eta^{\alpha}=\eta_{i}\left(t_{i} t_{j} \eta_{j}\right) \eta^{\alpha-\epsilon_{i}}-\left(t_{j} \eta_{j}\right) \eta^{\alpha-\epsilon_{i}} \\
= & \eta_{i}\left(t_{i} t_{j} \eta_{j}\right) \eta^{\alpha-\epsilon_{i}}-\eta^{\alpha-\epsilon_{i}}\left(t_{j} \eta_{j}\right)+\alpha_{j} \eta^{\alpha-\epsilon_{i}} \\
= & \eta_{i}^{\alpha_{i}}\left(t_{i} t_{j} \eta_{j}\right) \eta^{\alpha-\alpha_{i} \epsilon_{i}}-\alpha_{i} \eta^{\alpha-\epsilon_{i}}\left(t_{j} \eta_{j}\right)+\alpha_{i} \alpha_{j} \eta^{\alpha-\epsilon_{i}} \\
= & \eta_{i}^{\alpha i_{i}} \eta_{j}\left(t_{i} t_{j} \eta_{j}\right) \eta^{\alpha-\alpha_{i} \epsilon_{i}-\epsilon_{j}}-\eta_{i}^{\alpha}\left(t_{i} \eta_{j}\right) \eta^{\alpha-\alpha_{i} \epsilon_{i}-\epsilon_{j}}-\alpha_{i} \eta^{\alpha-\epsilon_{i}}\left(t_{j} \eta_{j}\right)+\alpha_{i} \alpha_{j} \eta^{\alpha-\epsilon_{i}} \\
= & \eta^{\alpha}\left(t_{i} t_{j} \eta_{j}\right)-\alpha_{j} \eta^{\alpha-\epsilon_{j}}\left(t_{i} \eta_{j}\right)-\alpha_{i} \eta^{\alpha-\epsilon_{i}}\left(t_{j} \eta_{j}\right)+\alpha_{i} \alpha_{j} \eta^{\alpha-\epsilon_{i}}
\end{aligned}
$$

The cases when $\alpha_{i}=0$ or $\alpha_{j}=0$ are similar, just skipping some steps. In any case will obtain:

$$
\left(t_{i} t_{j} \eta_{j}\right) \eta^{\alpha}=\eta^{\alpha}\left(t_{i} t_{j} \eta_{j}\right)+\alpha_{i} \alpha_{j} \eta^{\alpha-\epsilon_{i}}-\alpha_{j} \eta^{\alpha-\epsilon_{j}}\left(t_{i} \eta_{j}\right)-\alpha_{i} \eta^{\alpha-\epsilon_{i}}\left(t_{j} \eta_{j}\right)
$$

Let $v \in R_{k}(U)$ be a singular vector, that is, $\mathcal{V}(1) v=0$ and $v \notin R_{0}(U)$. For the next 
lemma we will use $\eta_{i}$ as in Lemma 29 with $l=k+4$.

Lemma 48. For $v$ a singular vector written as in (4.2), we have the following $\mathfrak{g l}_{N^{-}}$actions, if $\alpha_{i} \neq 0$ or $\alpha_{j}>1$ :

$$
\begin{array}{ll}
E_{i i} u_{\alpha}=\frac{1}{2}\left(\alpha_{i}-1\right) u_{\alpha}, & \\
E_{i j} u_{\alpha}=\frac{1}{2}\left(\alpha_{i}+1\right) u_{\alpha+\epsilon_{i}-\epsilon_{j}}, & \text { if } \alpha_{j} \neq 0, \\
E_{i j} u_{\alpha}=0, & \text { if } \alpha_{j}=0 .
\end{array}
$$

Proof. Applying $t_{i}^{2} \eta_{j}$ to $v$ and using equation (4.3), we obtain:

$$
\left(t_{i}^{2} \eta_{j}\right) v=\sum_{|\alpha| \leq k}\left(t_{i}^{2} \eta_{j}\right) \eta^{\alpha} \otimes u_{\alpha}=\sum_{|\alpha| \leq k}\left(\alpha_{i}\left(\alpha_{i}-1\right) \eta^{\alpha-2 \epsilon_{i}+\epsilon_{j}} \otimes u_{\alpha}-2 \alpha_{i} \eta^{\alpha-\epsilon_{i}} \otimes E_{i j} u_{\alpha}\right) .
$$

Since $v$ is a singular vector, this expression is equal to zero and we have:

$$
\sum_{|\alpha| \leq k} \alpha_{i}\left(\alpha_{i}-1\right) \eta^{\alpha-2 \epsilon_{i}+\epsilon_{j}} \otimes u_{\alpha}=\sum_{|\alpha| \leq k} 2 \alpha_{i} \eta^{\alpha-\epsilon_{i}} \otimes E_{i j} u_{\alpha}
$$

If $i=j$, comparing the coefficients of each $\eta^{\alpha}$ we obtain:

$$
\frac{1}{2} \alpha_{i}\left(\alpha_{i}-1\right) u_{\alpha}=\alpha_{i} E_{i i} u_{\alpha}
$$

If $i \neq j$, we make a shift on the left side of (4.5) sending $\alpha_{i}$ to $\alpha_{i}+1$ and $\alpha_{j}$ to $\alpha_{j}-1$ and, setting $u_{\alpha}$ to be zero if any coordinates is negative, we have:

$$
\sum_{|\alpha|=k}\left(\alpha_{i}+1\right) \alpha_{i} \eta^{\alpha-\epsilon_{i}} \otimes u_{\alpha+\epsilon_{i}-\epsilon_{j}}=2 \alpha_{i} \eta^{\alpha-\epsilon_{i}} \otimes E_{i j} u_{\alpha}
$$

Comparing the coefficients of each $\eta^{\alpha}$, we obtain:

$$
\frac{1}{2} \alpha_{i}\left(\alpha_{i}+1\right) u_{\alpha+\epsilon_{i}-\epsilon_{j}}=\alpha_{i} E_{i j} u_{\alpha}
$$

For $\alpha_{i} \neq 0$ these expressions restrict the possible $\mathfrak{g l}_{N^{-}}$actions as follows

$$
\begin{array}{ll}
E_{i i} u_{\alpha}=\frac{1}{2}\left(\alpha_{i}-1\right) u_{\alpha}, & \\
E_{i j} u_{\alpha}=\frac{1}{2}\left(\alpha_{i}+1\right) u_{\alpha+\epsilon_{i}-\epsilon_{j}}, & \text { if } \alpha_{j} \neq 0, \\
E_{i j} u_{\alpha}=0, & \text { if } \alpha_{j}=0 .
\end{array}
$$

This completes the claim of the lemma for one type of restriction. To prove the lemma in its generality, we will do the same thing now with $t_{i} t_{j} \eta_{j}$.

Applying $t_{i} t_{j} \eta_{j}$ to $v$ and using (4.4), we get

$$
\left(t_{i} t_{j} \eta_{j}\right) v=\sum_{|\alpha| \leq n}\left(\alpha_{i} \alpha_{j} \eta^{\alpha-\epsilon_{i}} \otimes u_{\alpha}-\alpha_{j} \eta^{\alpha-\epsilon_{j}} \otimes E_{i j} u_{\alpha}-\alpha_{i} \eta^{\alpha-\epsilon_{i}} \otimes E_{j j} u_{\alpha}\right)
$$

which implies

$$
\sum_{|\alpha| \leq n} \alpha_{i} \alpha_{j} \eta^{\alpha-\epsilon_{i}} \otimes u_{\alpha}=\sum_{|\alpha| \leq n}\left(\alpha_{j} \eta^{\alpha-\epsilon_{j}} \otimes E_{i j} u_{\alpha}+\alpha_{i} \eta^{\alpha-\epsilon_{i}} \otimes E_{j j} u_{\alpha}\right) .
$$


We have two ways to apply the shift in order to compare the coefficients at $\eta_{\alpha}$ :

$$
\alpha_{i} \alpha_{j} u_{\alpha}=\left(\alpha_{j}+1\right) E_{i j} u_{\alpha-\epsilon_{i}+\epsilon_{j}}+\alpha_{i} E_{j j} u_{\alpha}
$$

and

$$
\left(\alpha_{i}+1\right)\left(\alpha_{j}-1\right) u_{\alpha+\epsilon_{i}-\epsilon_{j}}=\alpha_{j} E_{i j} u_{\alpha}+\left(\alpha_{i}+1\right) E_{j j} u_{\alpha+\epsilon_{i}-\epsilon_{j}} .
$$

For the first, let $\alpha_{j}=0$, we have

$$
E_{i j} u_{\alpha-\epsilon_{i}+\epsilon_{j}}=-\alpha_{i} E_{j j} u_{\alpha}
$$

If $\alpha_{i}>1$, we can use (4.6) so the last equation becomes:

$$
E_{j j} u_{\alpha}=-\frac{1}{2} u_{\alpha}
$$

Since $i$ was arbitrary, we have that the previous formula $E_{j j} u_{\alpha}=\frac{1}{2}\left(\alpha_{j}-1\right) u_{\alpha}$ holds even if $\alpha_{j}=0$ as long as at least one $\alpha_{i}$ is greater than one.

For the second, for $\alpha_{i}=0$, we have

$$
\left(\alpha_{j}-1\right) u_{\alpha+\epsilon_{i}-\epsilon_{j}}=\alpha_{j} E_{i j} u_{\alpha}+E_{j j} u_{\alpha+\epsilon_{i}-\epsilon_{j}} .
$$

If $\alpha_{j}>1$, we get

$$
E_{i j} u_{\alpha}=\frac{1}{2}\left(\alpha_{i}+1\right) u_{\alpha+\epsilon_{i}-\epsilon_{j}}
$$

This implies that the second equation on (4.6) is still true if $\alpha_{i}=0$ as long $\alpha_{j}$ is greater than one. At this point, we also would like to record the case $\alpha_{j}=1$, which will be used latter:

$$
E_{i j} u_{\alpha}=-E_{j j} u_{\alpha+\epsilon_{i}-\epsilon_{j}}
$$

The first equation of the previous lemma implies that for $\alpha_{i} \neq 0 \neq \alpha_{j}$ or $\alpha_{k}>1$, for some $k$,

$$
\left(E_{i i}-E_{j j}\right) u_{\alpha}=\frac{1}{2}\left(\alpha_{i}-\alpha_{j}\right) u_{\alpha}
$$

The second and third equations of the previous lemma, for $\alpha_{i}$ and $\alpha_{j}$ non-zero or one of them greater than 1 , imply

$$
\left(E_{i j} E_{j i}-E_{j i} E_{i j}\right) u_{\alpha}=\frac{1}{4}\left(\alpha_{i}-\alpha_{j}\right) u_{\alpha}
$$

Proposition 49. Any singular vector in $R_{p}(U)$ belongs to $R_{1}(U)$.

Proof. Let $k \in \mathbb{Z}_{+}$such that $v \in R_{k}(U) \backslash R_{k-1}(U)$ and write $v$ as in (4.2). There exists a non-zero $u_{\alpha}$ with $|\alpha|=k$.

Suppose $k>1$, then there exist $i, j$ distinct, such that $\alpha_{i} \neq 0 \neq \alpha_{j}$ or there exists $i$ with $\alpha_{i}>1$. In both cases, expressions (4.8) and (4.9) are valid and in order to have a compatible $\mathfrak{g l}_{N^{-}}$-action we must have $\alpha_{i}=\alpha_{j}$.

However, the same would apply to $u_{\alpha-\epsilon_{i}+\epsilon_{j}}$ which is non-zero, because $E_{i j} u_{\alpha-\epsilon_{i}+\epsilon_{j}}=$ $\frac{1}{2} \alpha_{i} u_{\alpha}$ is non-zero. Then expressions (4.8) and (4.9) are not valid, and this is a contradiction to our hypothesis of $k>1$. 
This last lemma assures that singular vectors can only live on the first level below the top. This first level $R_{1}(U) / R_{0}(0)$ is a $\mathcal{V}_{+} / \mathcal{V}(1) \simeq \mathfrak{g l}_{N^{-}}$module. We will use the $\mathfrak{g l}_{N^{-}}$ module generated by the singular vectors to establish restrictions on $U$ resulting from the existence of singular vectors.

Theorem 50. If $U$ is a simple $\mathfrak{g l}_{N}$-module that is not the dual of an exterior power of the natural module, then the Rudakov module $R_{p}(U)$ is irreducible as a $\mathcal{V}$-module.

Proof. Let us consider the $\mathfrak{g l}_{N}$ module generated by the singular vectors. It is a finitedimensional module, so there exists a maximum weight vector with respect to the root system of $\mathfrak{g l}_{N}$. Let $v$ be a singular vector that is also a $\mathfrak{g l}_{N}$ highest weight vector. Then we have

$$
\left(t_{i} \eta_{j}\right) v=\sum_{k}\left(t_{i} \eta_{j}\right) \eta_{k} \otimes u_{k}=\sum_{k}\left(\eta_{k} \otimes E_{i j} u_{k}\right)-\eta_{j} \otimes u_{i}
$$

For $i<j$ we have $t_{i} \eta_{j} v=0$, which implies $E_{i j} u_{k}=\delta_{j k} u_{i}$. So if $u_{k}$ is zero then all $u_{i}$ with $i<k$ will also be zero.

Let $j$ be such that $u_{j}$ is non-zero but all $u_{i}=0$ for $i<j$. By the previous formula we have that $u_{j}$ is a highest weight vector for $U$. From the second equation of (4.6), we already have $E_{j j} u_{j}=0$. Equation (4.7) implies

$$
E_{i i} u_{j}=-E_{j i} u_{i}
$$

If $i<j, E_{i j}$ will be acting as zero since $u_{i}=0$, so $E_{i i} u_{j}=0$. If $i>j, E_{i i} u_{j}=-E_{j i} u_{i}=$ $-u_{j}$. So the weight of $u_{j}$ is equal to a fundamental weight and the identity is acting by $-j$. An irreducible finite-dimensional $\mathfrak{g l}_{N}$-module is uniquely determined by the weight of a highest weight vector and the action of the identity. So, once more, we have that there are only $N+1 \mathfrak{g l}_{N}$-modules that $R_{p}(U)$ could be reducible. The modules with such highest weights are the dual of an exterior power of the natural module: $\left(\Lambda^{j}\left(\mathbb{K}^{N}\right)\right)^{*}$.

In conclusion, the existence of a singular vector implies that $U$ is $\left(\Lambda^{k}\left(\mathbb{K}^{N}\right)\right)^{*}$.

\subsection{Tensor Product of Rudakov Modules}

For a given finite-dimensional simple Lie algebra $\mathfrak{g}$, one way to obtain an infinitedimensional Lie algebra is by taking its tensor product with $\mathbb{K}\left[t, t^{-1}\right]$. The commutator will then become

$$
[p \otimes g, q \otimes h]=f g \otimes[g, h] .
$$

This construction is known as the loop algebra. If instead we take the tensor product with $\mathbb{K}\left[t_{1}^{ \pm 1}, \ldots, t_{k}^{ \pm}\right]$we also obtain a Lie algebra known as the multiloop algebra.

The classification of all finite-dimensional simple modules for the loop algebras was given by Chari and Pressley [11]. For the multiloop algebras, the classification was done by Lau [25]. He proved that every finite-dimensional simple module for a multiloop algebra is isomorphic to a tensor product of evaluation modules. An evaluation module has two parameters: a weight $\lambda$ for $\mathfrak{g}$ and a point $a \in \mathbb{K}^{* k}$, it is the highest weight module $L(\lambda)$ with the action done by evaluating any element of the multiloop algebra on $a$ and then letting the resulting element of $\mathfrak{g}$ act on $L(\lambda)$.

His theorem motivated us to study the tensor product of Rudakov modules. That is what we describe in this section.

Let $p_{1}, \ldots, p_{m}$ be distinct points in $X$ and $U_{1}, \ldots, U_{m}$ be $\mathfrak{g l}_{N}-$ modules, none of them isomorphic to any $\left(\Lambda^{k}\left(\mathbb{K}^{N}\right)\right)^{*}$. We already know that each $R_{p_{j}}\left(U_{j}\right)$ is an irreducible 
$\mathcal{V}$-module. We want to show that the tensor product $R:=R_{p_{1}}\left(U_{1}\right) \otimes \ldots \otimes R_{p_{m}}\left(U_{m}\right)$ is also an irreducible $\mathcal{V}$-module. In what follows we will write $\mathcal{V}_{i}(l)$ for the filtration around each $p_{i}$. The following lemma will be our main tool:

Lemma 51. Given $p_{1}, \ldots, p_{m} \in X$ distinct and $s_{2}, \ldots, s_{m} \in \mathbb{Z}_{+}$, there exists $g \in \mathfrak{m}_{p_{2}}^{s_{2}} \times$ $\ldots \times \mathfrak{m}_{p_{n}}^{s_{n}}$ such that $g\left(p_{1}\right)=1$.

Proof. It is well known that powers of co-primes ideals are also co-prime. So $\mathfrak{m}_{p_{1}}$ and $\mathfrak{m}_{p_{i}}^{s_{i}}$, for any $i>1$, are co-prime. Let $f_{i} \in \mathfrak{m}_{p_{i}}^{s_{i}}$ and $f_{i}^{\prime} \in \mathfrak{m}_{p_{1}}$ be such that $f_{i}+f_{i}^{\prime}=1$, let $g=\prod_{i=2}^{n} f_{i}$ then $g \in \mathfrak{m}_{p_{2}}^{s_{2}} \times \ldots \times \mathfrak{m}_{p_{n}}^{s_{n}}$. Since $f_{i}\left(p_{1}\right)=1$ we have $g\left(p_{1}\right)=1$.

For $v \in R$, there exist $s_{1}, \ldots, s_{m}$ such that $v \in R_{s_{1}}\left(U_{1}\right) \otimes \ldots \otimes R_{s_{m}}\left(U_{m}\right)$ but $v \notin$ $R_{s_{1}-1}\left(U_{1}\right) \otimes R_{s_{2}}\left(U_{2}\right) \otimes \ldots \otimes R_{s_{m}}\left(U_{m}\right)$. Let $\left\{w_{k}^{j}\right\}_{k}$ be a basis for $R_{s_{j}}\left(U_{j}\right)$, for $j \in\{2, \ldots, m\}$. Then $v$ can be written as

$$
v=\sum_{k_{2}, \ldots, k_{m}} v_{\left(k_{2}, \ldots, k_{m}\right)} \otimes w_{k_{2}}^{2} \ldots \otimes w_{k_{m}}^{m}
$$

Proposition 52. The $\mathcal{V}$-submodule generated by $v$ intersects $U_{1} \otimes \ldots \otimes U_{m}$ non-trivialy.

Proof. By Lemma 51 (a) there exists $g \in \mathfrak{m}_{p_{2}}^{s_{2}+1} \times \ldots \times \mathfrak{m}_{p_{n}}^{s_{n}+1}$ with $g\left(p_{1}\right)=1$, By Lemma 29 for any $\eta \in \mathcal{V}_{1}(1)$ we have

$$
(g \eta) v=\sum_{k_{2}, \ldots, k_{m}} \eta v_{\left(k_{2}, \ldots, k_{m}\right)} \otimes w_{k_{2}}^{2} \ldots \otimes w_{k_{m}}^{m}+v_{0}
$$

where $v_{0} \in R_{s_{1}-2}\left(U_{1}\right) \otimes R_{s_{2}}\left(U_{2}\right) \otimes \ldots \otimes R_{s_{m}}\left(U_{m}\right)$, this follows from the fact that if $g_{0}$ is the constant term of $g$ when we write it in terms of standard chart parameters centered at $p_{1}$, since $g\left(p_{1}\right)=1$ we have $g_{0}=1$ and $g \eta \in \mathcal{V}_{i}\left(s_{i}+1\right)$ for all $i \in\{2, \ldots, m\}$.

Let us take $\left(k_{2}^{\prime}, \ldots, k_{m}^{\prime}\right)$ such that $v_{\left(k_{2}^{\prime}, \ldots, k_{m}^{\prime}\right)} \in R_{s_{1}} \backslash R_{s_{1}-1}$ and since $R_{p_{1}}\left(U_{1}\right)$ has no singular vectors there exist $\eta_{1}, \ldots, \eta_{s_{1}} \in \mathcal{V}(1)$ such that $\eta_{s} \ldots \eta_{1} v_{\left(k_{2}^{\prime}, \ldots, k_{m}^{\prime}\right)} \in U_{1}$ and is non-zero. Then

$$
g \eta_{s} \ldots g \eta_{1} v=\sum_{k_{2}, \ldots, k_{m}} \eta_{s} \ldots \eta_{1} v_{\left(k_{2}, \ldots, k_{m}\right)} \otimes w_{k_{2}}^{2} \ldots \otimes w_{k_{m}}^{m}
$$

is a non-zero vector in $U_{1} \otimes R_{p_{2}}\left(U_{2}\right) \otimes R_{p_{m}}\left(U_{m}\right)$.

We can apply the same argument to each coordinate to show that we can obtain from any vector in the tensor product a vector in $U_{1} \otimes \ldots \otimes U_{m}$.

The tensor product of irreducible $\mathfrak{g l}_{N}$-modules does not need to be irreducible. However for $\mathcal{V}_{+}$-modules corresponding to distinct points on $X$, this is not the case:

Proposition 53. $U_{1} \otimes \ldots \otimes U_{m}$ is an irreducible $\mathcal{V}_{+}$-module.

Proof. We can apply any $\mathfrak{g l}_{N}$-action to any coordinate without changing the others. For example, if we want to apply $E_{i j}$ to the first coordinate only, we once again just need to multiply the vector field we want to act by $g \in \mathfrak{m}_{p_{2}} \times \ldots \times \mathfrak{m}_{p_{m}}$ from Lemma 51 , that is, we apply $g t_{i} \eta_{j}$, once again it will acts as zero in all coordinates except the first where it will be applying $E_{i j}$ plus terms belonging to $\mathcal{V}_{1}(2)$, which will act as zero. 
Now, if we can apply any element of $\mathfrak{g l}_{N}$ to any component we may use the Vandermonde argument to show that we have the decomposition in terms of weights for each $U_{i}$. Since each $U_{i}$ is irreducible, from any element of the form

$$
u_{1} \otimes \ldots \otimes u_{k}
$$

we can obtain the whole $U_{1} \otimes \ldots \otimes U_{k}$.

Now we are in position to prove the main theorem of this section:

Theorem 54. Let $p_{1}, \ldots, p_{m} \in X$ and $U_{1}, \ldots, U_{m}$ be $\mathfrak{g l}_{N}$-modules, none of them isomorphic to any $\left(\Lambda^{k}\left(\mathbb{K}^{N}\right)\right)^{*}$. Then $R_{p_{1}}\left(U_{1}\right) \otimes \ldots \otimes R_{p_{m}}\left(U_{m}\right)$ is irreducible as $\mathcal{V}$-module.

Proof. Let $v \in R$ then there exist $s_{1}, \ldots, s_{m}$ such that $v \in R_{s_{1}}\left(U_{1}\right) \otimes \ldots \otimes R_{s_{m}}\left(U_{m}\right)$. In what follows we will write $t_{i j}$ for the $j$-th chart parameter around $p_{i}$ and $\eta_{i j}$ will be as in (4.1) with the filtration been taken in terms of $\mathfrak{m}_{p_{i}}$ and $l_{i}=s_{i}$. Let $R_{i} \subset R, i \in \mathbb{N}$, be the vector space generated by elements of the form:

$$
\eta_{1}^{\alpha_{1}} u_{1} \otimes \ldots \otimes \eta_{m}^{\alpha_{m}} u_{m}
$$

with $\left|\alpha_{1}\right|+\ldots+\left|\alpha_{m}\right|=i$ and $u_{i} \in U_{i}$, so $R_{0}=U_{1} \otimes \ldots \otimes U_{m}$.

We want to show that $R_{0}$ generates $R$. To do so, we will prove by induction on $i$ that $R_{i}$ is contained in the module $\mathcal{U}(\mathcal{V}) R_{0}$. The basis of induction being $R_{0} \subset \mathcal{U}(\mathcal{V}) R_{0}$. Now suppose that, for a given $i$, we have $R_{i-1} \subset \mathcal{U}(\mathcal{V}) R_{0}$. For the induction step we need to show that given any element like (4.10), with $\left|\alpha_{1}\right|+\ldots+\left|\alpha_{m}\right|=i$, we can obtain it from $R_{0}$. Let us take $u:=u_{1} \otimes \ldots \otimes u_{m}$ and let us take, from Lemma 51, $g_{j} \in \mathfrak{m}_{p_{1}}^{\left|\alpha_{1}\right|+1} \otimes \ldots \otimes \mathfrak{m}_{p_{j-1}}^{\left|\alpha_{j-1}\right|+1} \otimes \mathfrak{m}_{p_{j+1}}^{\left|\alpha_{j+1}\right|+1} \otimes \ldots \otimes \mathfrak{m}_{p_{m}}^{\left|\alpha_{m}\right|+1}$ such that $g_{j}\left(p_{j}\right)=1$. For the first coordinate we have:

$$
\underbrace{g_{1} \eta_{11} \ldots g_{1} \eta_{11}}_{\alpha_{11} \text { times }} \cdots \underbrace{g_{1} \eta_{1 N} \ldots g_{1} \eta_{1 N}}_{\alpha_{1 N} \text { times }} u=\eta_{1}^{\alpha_{1}} u_{1} \otimes u_{2} \ldots \otimes u_{m}+w_{j}
$$

where $w_{j} \in R_{j}$. If we do the same process to each coordinate we will obtain the vector (4.10) plus some element in $R_{j}$ which we supposed to be in $\mathcal{U}(\mathcal{V}) R_{0}$, so $R_{i+1}$ is also in $\mathcal{U}(\mathcal{V}) R_{0}$. In particular, $v \in R_{s_{1}+\ldots+s_{m}} \subset \mathcal{U}(\mathcal{V}) R_{0}$.

So we have shown, by the Proposition 52, that any vector generates a submodule intersecting $R_{0}$ non-trivially which, by Proposition 53 is irreducible as $\mathcal{V}_{+}$-module, and now we have shown that $R_{0}$ generates the whole $R$. 


\section{Chapter 5}

\section{Realizations of de Rham Complex}

So far we discussed the problem of determing irreducibility of representations. Another problem of representation theory is the problem of classifying such representations and finding realizations, we have briefly discussed this in section 3.4.

There is a generalization of weight modules called Gelfand-Tsetlin modules where, roughly speaking, the Cartan subalgebra acts by Jordan blocks. For this class of modules there is a classification, due to Futorny, Gratcharov and Ramirez [14], for $\mathfrak{g l}_{3}$.

Harish-Chandra's theorem characterizes finite-dimensional modules by looking at the center of the universal enveloping algebra. The classification present in [14] uses a similar idea, they focus on a subalgebra of $\mathcal{U}\left(\mathfrak{g l}_{N}\right)$, the center of the universal enveloping algebra of copies of $\mathfrak{g l}_{m}, m \leq N$, inside $\mathfrak{g l}_{N}$.

In this appendix we will present $\mathfrak{g l}_{N+1}$-modules by restricting the action on the $\mathcal{V}_{\mathbb{T}^{N^{-}}}$ module $q^{\gamma} \Omega^{k}\left(\mathbb{T}^{N}\right)$ that appeared in Theorem 32. This module is already reducible as $\mathcal{V}_{\mathbb{T}^{N}}$-module, but some irreducible components reduce further as $\mathfrak{g l}_{N+1}$-module when the action is restricted. We also determined how they fit into the classification of FurtornyGrantcharov-Ramirez for $N=2$.

\section{$5.1 \mathfrak{g l}_{N+1}$-modules from $\mathcal{V}_{\mathbb{T}^{N}}$-module}

We will be using the notation of section 3.1, refer to that section for more details. Let $\mathcal{V}_{\mathbb{T}^{N}}$ be the Lie algebra of vector fields on an $N$ dimensional torus, $d_{p}$ be the vector fields $t_{p} \frac{\partial}{\partial t_{p}}$ and $T(U, \gamma)$ be the tensor module as defined in section 3.1.

There exists an embedding of $\mathfrak{g l}_{N+1}$ in $\mathcal{V}_{\mathbb{T}^{N}}$ given as follows:

$$
\begin{gathered}
E_{i j} \rightarrow t_{i} t_{j}^{-1} d_{j}, \\
E_{i, N+1} \rightarrow-t_{i} D, \quad E_{N+1, i}=t_{i}^{-1} d_{i}, \\
E_{N+1, N+1} \rightarrow-D,
\end{gathered}
$$

where $1 \leq i, j \leq N$, and $D:=\sum_{i=1}^{N} d_{i}$.

Using this embedding the tensor modules $T(U, \gamma)$ become $\mathfrak{g l}_{N+1}$-modules with the action given as follows:

$$
E_{i j}\left(q^{\mu} \otimes v\right)=\mu_{j} q^{\mu+\epsilon_{i}-\epsilon_{j}} \otimes v+q^{\mu+\epsilon_{i}-\epsilon_{j}} \otimes\left(\bar{E}_{i j}-\bar{E}_{j j}\right) v
$$




$$
\begin{gathered}
E_{i, N+1}\left(q^{\mu} \otimes v\right)=-\left(\sum_{j} \mu_{j} q^{\mu+\epsilon_{i}} \otimes v+q^{\mu+\epsilon_{i}} \otimes \sum_{j} \bar{E}_{i j} v\right), \\
E_{N+1, i}\left(q^{\mu} \otimes v\right)=\mu_{i} q^{\mu-\epsilon_{i}} \otimes v-q^{\mu-\epsilon_{i}} \otimes \bar{E}_{i i} v, \\
E_{N+1, N+1}\left(q^{\mu} \otimes v\right)=-\sum_{i} \mu_{i} q^{\mu} \otimes v .
\end{gathered}
$$

The $\mathfrak{g l}_{N}$ action on $U$ is denoted with an overbar.

In [28], it was also proved that $d^{k}\left(T\left(\Lambda^{k} \mathbb{C}^{N}, \gamma\right)\right)$ is an irreducible $\mathcal{V}_{\mathbb{T}^{N}}$-module. In what follows, we will find conditions for these modules to be irreducible as $\mathfrak{g l}_{N}$-modules.

The space $d^{0}\left(T\left(\Lambda^{0} \mathbb{C}^{N}, \gamma\right)\right) \subset T\left(\mathbb{C}^{N}, \gamma\right)$ is generated by the set $\left\{q^{\mu} \otimes \mu \mid \mu \in \mathbb{Z}+\gamma\right\}$. Applying the $\mathfrak{g l}_{N+1}$-action to these vectors we obtain:

$$
\begin{gathered}
E_{i j}\left(q^{\mu} \otimes \mu\right)=\mu_{j} q^{\mu+\epsilon_{i}-\epsilon_{j}} \otimes\left(\mu+\epsilon_{i}-\epsilon_{j}\right), \\
E_{i, N+1}\left(q^{\mu} \otimes \mu\right)=-\sum_{j} \mu_{j} q^{\mu+\epsilon_{i}} \otimes\left(\mu+\epsilon_{i}\right), \\
E_{N+1, i}\left(q^{\mu} \otimes \mu\right)=\mu_{i} q^{\mu-\epsilon_{i}} \otimes\left(\mu-\epsilon_{i}\right), \\
E_{N+1, N+1}\left(q^{\mu} \otimes \mu\right)=-\sum_{i} \mu_{i} q^{\mu} \otimes \mu .
\end{gathered}
$$

Example: Let us consider the case $N=2$. The weight spaces of $d^{0}\left(T\left(\Lambda^{0} \mathbb{C}^{2}, \gamma\right)\right)$ form a $\mathbb{Z}^{2}$ lattice, where each space is generated by $q^{\mu} \otimes \mu$, except for the case $\gamma \in \mathbb{Z}^{2}$ where we have a hole at the origin. Using the same notation as in the example on the page 19, we have the following diagram:

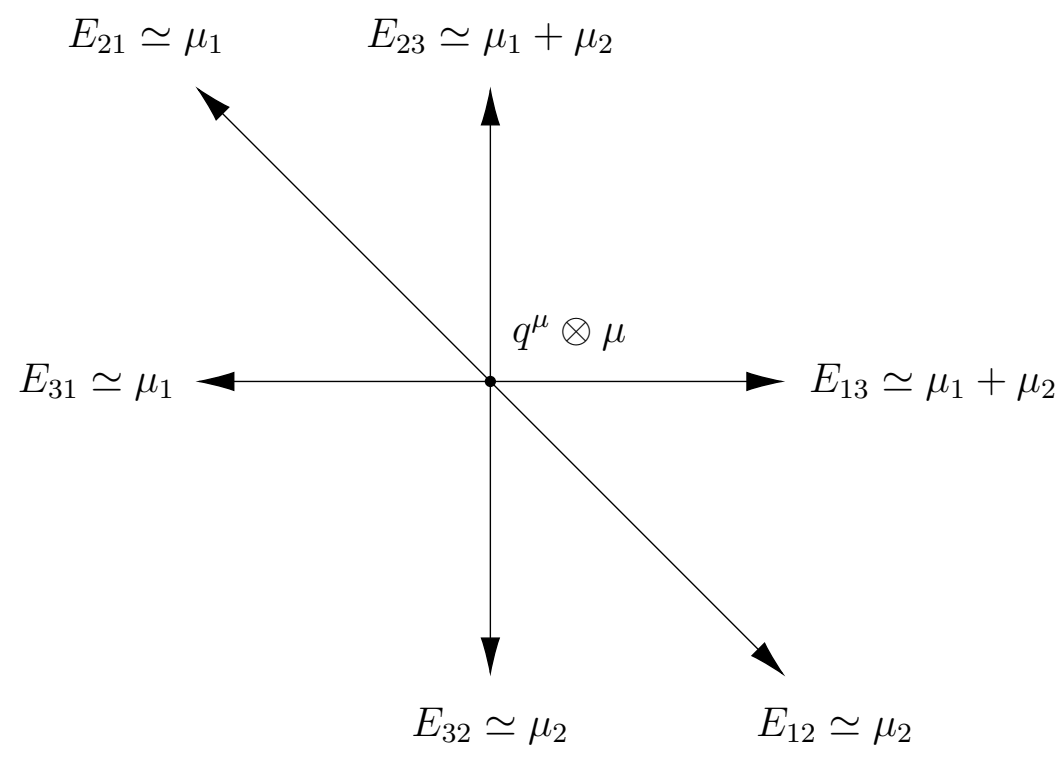

This diagram shows us that starting from a vector $q^{\mu} \otimes \mu$, we cannot reach a vector on the left if $\mu_{1}=0$, similarly we cannot move down if $\mu_{2}=0$ and at the line $\mu_{1}+\mu_{2}=0$ you cannot move up or left, except alongside it. When none of this happens we are free to go from one vector to any other vector on the lattice. 
Proposition 55. $\Xi:=d^{0}\left(T\left(\Lambda^{0} \mathbb{C}^{N}, \gamma\right)\right)$ is irreducible as $\mathfrak{g l}_{N+1}$-module if and only if $\sum_{j} \gamma_{j} \notin \mathbb{Z}$ and $\gamma_{i} \notin \mathbb{Z}$ for all $i \in\{1, \ldots, N\}$.

Proof. The weight spaces of $d^{0}\left(T\left(\Lambda^{0} \mathbb{C}^{N}, \gamma\right)\right)$ forms a $\mathbb{Z}^{N}$ lattice with each space of dimension 1 . Given any vector in $\Xi$, by the Vandermonde argument we have that $\Xi$ contains the weight decomposition of that vector. By the previous example, we can generalize it for any $N$ and we see that if $\sum_{j} \gamma_{j}$ and $\gamma_{i} \notin \mathbb{Z}$ for all $i \in\{1, \ldots, N\}$, then starting from any $q^{\mu} \otimes \mu$ we can reconstruct the whole module. On the other hand, if any of the conditions fail we will have some part of the module that is not reachable from the other. Indeed, if $\mu_{i} \in \mathbb{Z}$ then we cannot obtain a vector with $\mu_{i}$ negative from one with $\mu_{i}$ positive. Similarly, if $\sum_{j} \mu_{j} \in \mathbb{Z}$ we cannot obtain a vector with $\sum_{j} \mu_{j}$ positive from one with $\sum_{j} \mu_{j}$ negative. In all these cases $\Xi$ will be reducible.

We have 5 cases to consider, in order to present completely the decomposition of $\Xi$.

Case 1: $\gamma_{1}, \gamma_{2}, \gamma_{1}+\gamma_{2} \notin \mathbb{Z}$. In this case $\Xi$ is irreducible.

Case 2: $\gamma_{1} \in \mathbb{Z}$ and $\gamma_{2} \notin \mathbb{Z}$ : We have the following chain of submodules $\Xi_{1} \subset \Xi_{2}$, where $\Xi_{1}=\operatorname{Span}\left\{q^{\mu} \otimes \mu \mid \mu_{1} \geq 0\right\}$ and $\Xi_{2}=\Xi / \Xi_{1}$.

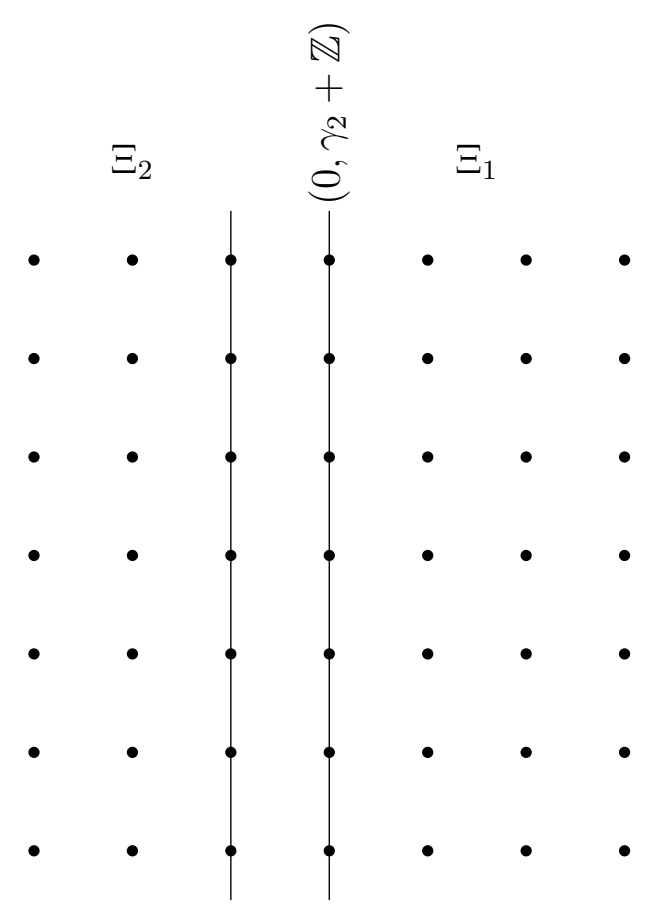


Case 3: $\gamma_{1} \notin \mathbb{Z}$ and $\gamma_{2} \in \mathbb{Z}$ : Similar to case 2 but with $\Xi=\operatorname{Span}\left\{q^{\mu} \otimes \mu \mid \mu_{2} \geq 0\right\}$.

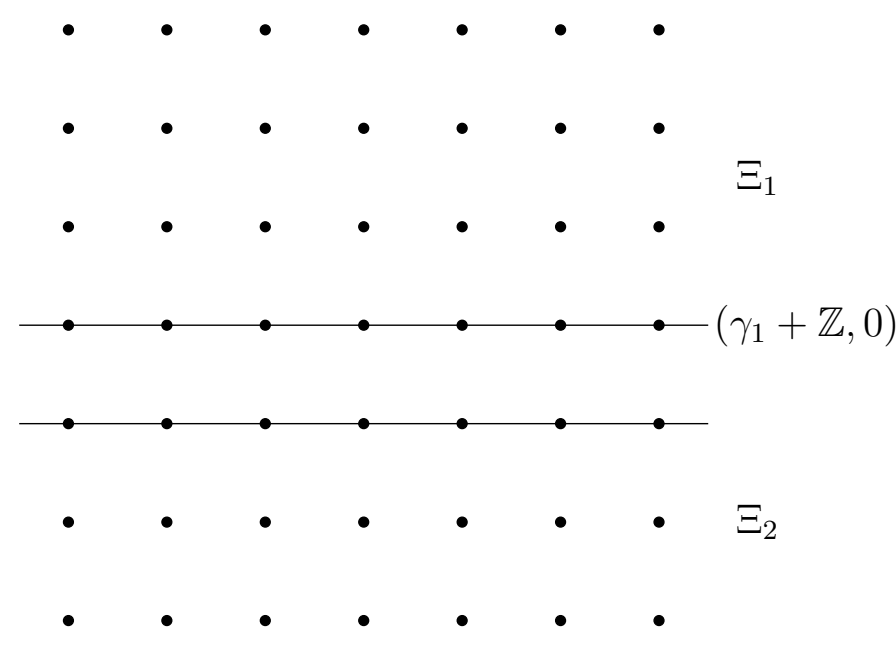

Case 4: $\gamma_{1}, \gamma_{2} \notin \mathbb{Z}$ and $\gamma_{1}+\gamma_{2} \in \mathbb{Z}$ : Similar to case 2 but with $\Xi=\operatorname{Span}\left\{q^{\mu} \otimes \mu \mid \mu_{1}+\right.$ $\left.\mu_{2} \leq 0\right\}$.

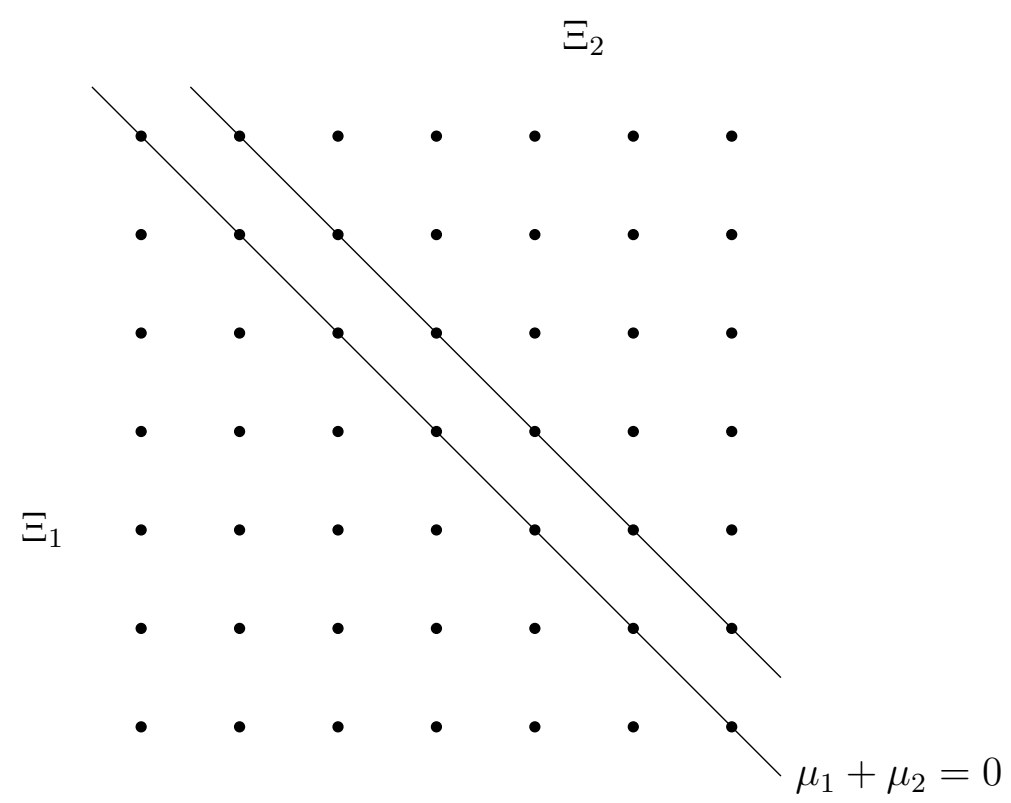

Case 5: $\gamma_{1} \gamma_{2} \in \mathbb{Z}$ : In this case we have three submodules $\Xi_{1}, \Xi_{2}$ and $\Xi_{3}$. Once we quotient by them we get three other submodules: $\Xi_{4}, \Xi_{5}$ and $\Xi_{6}$. They are described as follows:

$$
\begin{gathered}
\Xi_{1}=\operatorname{Span}\left\{q^{\mu} \otimes \mu \mid \mu_{1} \geq 0, \mu_{2} \geq 0, \mu_{1}+\mu_{2}>0\right\}, \\
\Xi_{2}=\operatorname{Span}\left\{q^{\mu} \otimes \mu \mid \mu_{1} \geq 0, \mu_{2}<0, \mu_{1}+\mu 2 \leq 0\right\}, \\
\Xi_{3}=\operatorname{Span}\left\{q^{\mu} \otimes \mu \mid \mu_{1}<0, \mu_{2} \geq 0, \mu_{1}+\mu 2 \leq 0\right\}, \\
\Xi_{4}=\operatorname{Span}\left\{q^{\mu} \otimes \mu \mid \mu_{1}>0, \mu_{1}+\mu 2>0\right\}, \\
\Xi_{5}=\operatorname{Span}\left\{q^{\mu} \otimes \mu \mid \mu_{2}<0, \mu_{1}+\mu 2>0\right\}, \\
\Xi_{6}=\operatorname{Span}\left\{q^{\mu} \otimes \mu \mid \mu_{1}<0, \mu_{2}<0\right\} .
\end{gathered}
$$

Using the standard $\mathbb{Z}^{2}$ lattice with the origin in the center, we have the following descrip- 
tion of $\Xi$ :

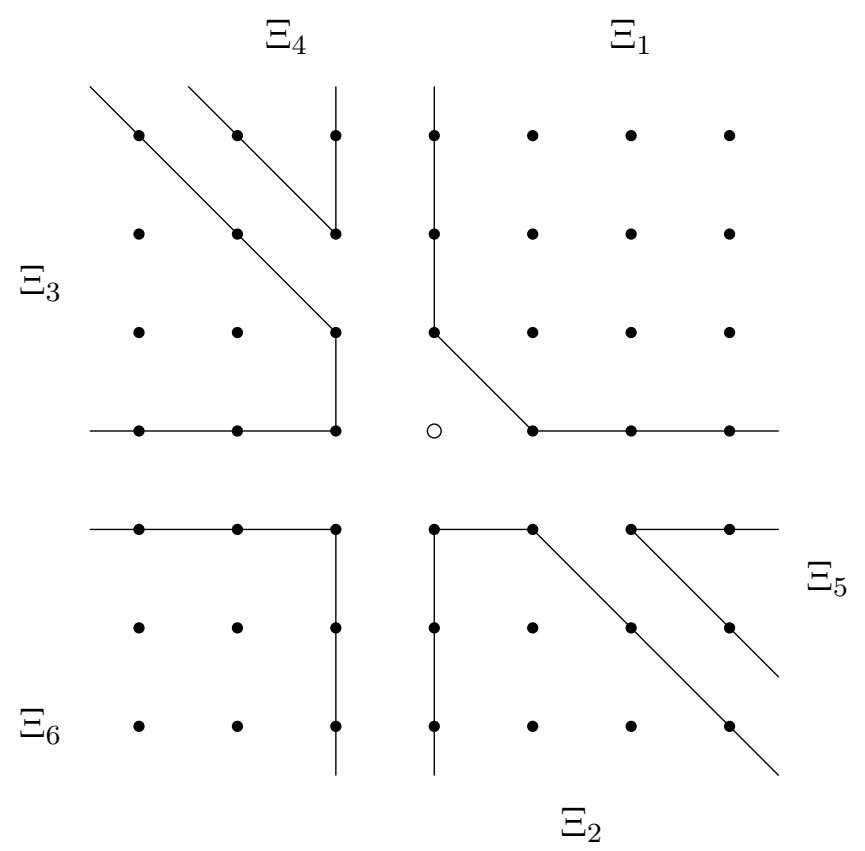

\subsection{Gelfand-Tsetlin-modules for $\mathfrak{g l}_{N}$}

Rao also proved that $T\left(\Lambda^{k} \mathbb{K}^{N}, \gamma\right) / d T\left(\Lambda^{k-1} \mathbb{K}^{N}, \gamma\right)$ is irreducible as $\mathcal{V}_{\mathbb{T}^{N} \text {-module. The }}$ machinery of the previous section is not suitable for this case. So, to give a complete description of the de Rham complex for $N=2$ we will resort to a different realization of $\mathfrak{g l}_{N}$-modules called Gelfand-Tsetlin tableaux and use the Futorny-Grancharov-Ramirez classification for $\mathfrak{g l}_{3}$.

Observe that $\left\{E_{i j}\right\}$ with $1 \leq i, j \leq s$, is a copy of $\mathfrak{g l}_{s}$ inside $\mathfrak{g l}_{N}$. This induces a chain of associative algebras

$$
\mathcal{U}\left(\mathfrak{g l}_{1}\right) \subset \ldots \subset \mathcal{U}\left(\mathfrak{g l}_{N}\right) .
$$

Let $Z_{m} \subset \mathcal{U}\left(\mathfrak{g l}_{N}\right)$ be the center of $\mathcal{U}\left(\mathfrak{g l}_{m}\right)$. We define $\Gamma \subset \mathcal{U}\left(\mathfrak{g l}_{N}\right)$ to be the algebra generated by all elements in $\bigcup_{m=1}^{N} Z_{m}$, it is called Gelfand-Tsetlin subalgebra or simply GT-subalgebra. With our choice of copies of $\mathfrak{g l}_{s}$ inside $\mathfrak{g l}_{N}$, this algebra is the polynomial algebra in the variables

$$
C_{m k}:=\sum_{\left(i_{1}, \ldots, i_{k}\right) \in\{1, \ldots, m\}^{k}} E_{i_{1} i_{2}} E_{i_{2} i_{3}} \ldots E_{i_{k} i_{1}} .
$$

Observe that for different choices of copies of $\mathfrak{g l}_{s}$ we obtain different GT-subalgebras. For example, for $\mathfrak{g l}_{3}$ there are three different GT-subalgebras.

Definition 56. A finitely generated $\mathfrak{g l}_{N}$-module $M$ is called a Gelfand-Tsetlin module (relative to $\Gamma$ ) if the restriction of the $\mathcal{U}\left(\mathfrak{g l}_{N}\right)$ to $\Gamma$ is a direct sum of $\Gamma$-modules:

$$
\left.M\right|_{\Gamma}=\bigoplus_{\mathfrak{m} \in \text { Specm } \Gamma} M(\mathfrak{m})
$$

where

$$
M(\mathfrak{m}):=\left\{v \in M \mid \mathfrak{m}^{k} v=0 \text { for some } k \geq 0\right\}
$$


Since $\Gamma$ contains the standard Cartan subalgebra, all weight modules are GT-modules, Indeed, $E_{i i}=C_{i 1}-C_{i-1,1}$ for $i>1$ and $E_{11}=C_{11}$.

An important tool in the realization of GT-modules is the following GT-tableau:

Definition 57. Let $v \in \mathbb{K}^{\frac{n(n+1)}{2}}$, then by $T(v)$ we denote the tableau

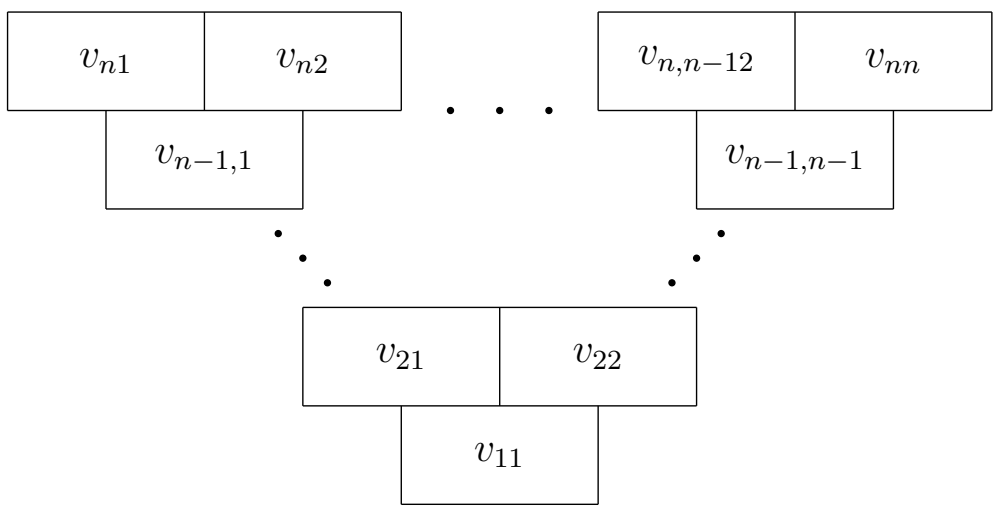

Such an array will be called a GT tableau of height $n$.

A GT tableau $T(v)$ of height $n$ is called:

- standard if $v_{k i}-v_{k-1, i} \in \mathbb{Z} \geq 0$ and $v_{k-1, i}-v_{k, i+1} \in \mathbb{Z}>0$ for all $1 \leq i \leq k \leq n-1$.

- generic if $v_{k i}-v_{k j} \notin \mathbb{Z}$ for all $1 \leq i \neq j \leq k \leq n-1$.

- singular if there exist $1 \leq s<t \leq r \leq n-1$ such that $v_{r s}-v_{r t} \in \mathbb{Z}$.

The $\mathfrak{g l}_{N}$-actions on standard tableaux are defined in [14, Theorem 4.4], for generic case (only $N=3$ ) see [14, Example 4.23] and for singular case see [14, Theorem 4.35].

For elements of $\Gamma$ the action on the tableaux is given by $C_{m k}(T(v))=\gamma_{m k}(v) T(v)$, where

$$
\gamma_{m k}(l)=\sum_{i=1}^{m}\left(l_{m i}+m-1\right)^{k} \prod_{j \neq i}^{m}\left(1-\frac{1}{l_{m i}-l_{m j}}\right) .
$$

\section{$5.3 \mathfrak{g l}_{3}$}

In this section we will focus on the case of $\mathfrak{g l}_{3}$ and we will describe completely the de Rham complex. Fix $\Gamma \subset \mathcal{U}\left(\mathfrak{g l}_{3}\right)$ as defined in the previous section, so for example, $C_{11}$ and $C_{21}$ generators of $\Gamma$ are giving by $C_{11}=E_{11}$ and $C_{21}=E_{11}+E_{22}$. Applying the generators of $\Gamma$ to a generic vector $q^{\mu} \otimes v$ of $T\left(\mathbb{K}^{2}, \gamma\right)$, we obtain $C_{11}=\mu_{1} \mathrm{Id}, C_{21}=\left(\mu_{1}+\mu_{2}\right) \mathrm{Id}, C_{31}=$ $C_{32}=C_{33}=0$ and

$$
C_{22} \simeq\left(\begin{array}{cc}
\left(\mu_{1}+\mu_{2}+1\right)\left(\mu_{1}+\mu_{2}\right) & 0 \\
0 & \left(\mu_{1}+\mu_{2}-1\right)\left(\mu_{1}+\mu_{2}\right)
\end{array}\right)
$$

Let $v \in \mathbb{K}^{6}$, solving the system of equations $C_{m k}(T(v))=\gamma_{m k}(v) T(v)$ we get $v_{11}=$ $\mu_{1}, v_{31}=0, v_{32}=-1$ and $v_{33}=-2$. For the second row of $v$, we have two possible solutions $v_{21}=\mu_{1}+\mu_{2}, v_{22}=-1$ and $v_{21}=0, l_{22}=\mu_{1}+\mu_{2}-1$. 
For a fixed $\mu \in \gamma+\mathbb{Z}^{2}$, we have any vector $q^{\mu} \otimes v$ when realized by GT-tableaux is written as a linear combination of the following tableaux:
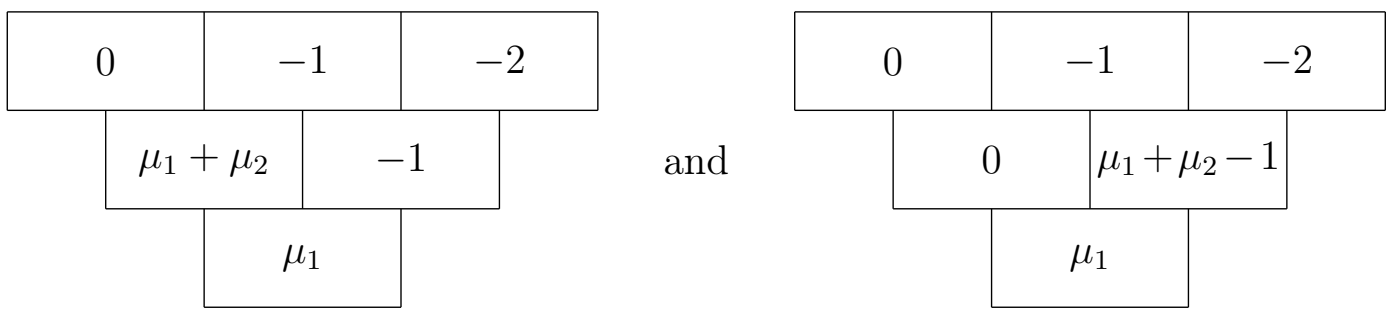

Applying $C_{22}$ to $q^{\mu} \otimes \mu$, we obtain that it is the eigenvector of the eigenvalue $\left(\mu_{1}+\right.$ $\left.\mu_{2}+1\right)\left(\mu_{1}+\mu_{2}\right)$. So the first tableau is related to $\Xi$ while the second is related to $T\left(\mathbb{K}^{2}, \gamma\right) / d T\left(\Lambda^{0} \mathbb{K}^{2}, \gamma\right)$.

Now we have all the tools to find these modules inside the Futorny-GrantcharovRamirez classification. Following the notation of [14], we have five cases to consider:

Case 1: $\gamma_{1}, \gamma_{2}$ and $\gamma_{1}+\gamma_{2} \notin \mathbb{Z}$ table will be in the format of G.16: $L_{2}$ and $L_{3}$.

Case 2: $\gamma_{1} \in \mathbb{Z}$ and $\gamma_{2} \notin \mathbb{Z}$ the table will be in the format of G.14: $L_{2}, L_{5}, L_{4}$ and $L_{7}$.

Case 3: $\gamma_{1} \notin \mathbb{Z}$ and $\gamma_{2} \in \mathbb{Z}$ table will be in the format of G.15: $L_{2}, L_{5}, L_{4}$ and $L_{7}$.

Case 4: $\gamma_{1}, \gamma_{2} \notin \mathbb{Z}$ and $\gamma_{1}+\gamma_{2} \in \mathbb{Z}$ table will be in the format of C.9: $L_{2}, L_{3}, L_{5}$ and $L_{8}$.

Case 5: $\gamma_{1} \in \mathbb{Z}$ and $\gamma_{2} \in \mathbb{Z}$ table will be in the format of C.10: $L_{9}, L_{14}, L_{21}, L_{26}, L_{22}$, $L_{15}, L_{7}, L_{11}, L_{16}, L_{24}, L_{17}$ and $L_{12}$. 
REALIZATIONS OF DE RHAM COMPLEX 


\section{Bibliography}

[1] M. Banados and I. Reyes; A short review on Noether's theorems, gauge symmetries and boundary terms; Int. J. Mod. Phys, D25 10, (2016) 1630021. 3

[2] V. V. Bavula; Generalized Weyl algebras and their representations; Algebra i Analiz, 4 (1992), 75-97. 41, 42

[3] Y. Billig; Jet modules; Canad. J. Math, 59 (2007), 712-729. 33, 44

[4] Y. Billig and V. Futorny; Representations of Lie algebra of vector fields on a torus and chiral de Rham complex; Trans. Amer. Math. Soc. 366 (2014) no. 9, 4697-4731. 7

[5] Y. Billig, V. Futorny; Classification of irreducible representations of Lie algebra of vector fields on a torus; J. Reine Angew. Math., 2016 (2016), 199-216. 3

[6] Y. Billig, V. Futorny; Lie algebras of vector fields on smooth affine varieties; Commun. in Algebra, 46 (2018), 3413-3429. 3, 26

[7] Y. Billig, V. Futorny, J. Nilsson; Representations of the Lie algebra of vector fields on affine varieties; arXiv:1709.08863 [math.RT]. 4, 28, 31, 33, 44

[8] Y. Billig, J. Nilsson; Representations of the Lie algebra of vector fields on a sphere; arXiv:1705.06685 [math.RT], to appear in J. Pure and Applied Algebra. 3, 26, 27

[9] R. E. Block; The irreducible representations of the Lie algebra sl(2) and of the Weyl algebra; Adv. Math. 39 (1981), 69-110. 2, 40

[10] E. Cartan; Sur la structure des groups des groups de transformations finis et continus; Thesis (Paris), (1894). 2

[11] V. Chari, A. Pressley; A new family of irreducible, integrable modules for affine Lie algebras; Math. Ann.,277 (1987), 543-562. 48

[12] E.B. Dynkin, Semisimple subalgebras of semisimple Lie algebras; Mat. Sb. (N.S.), 30(72) (1952) no. 2, 349-462. 12

[13] P. Etingof, O. Golberg, S. Hensel, T. Liu, A. Schwendner, D. Vaintrob, and E. Yudovina, Representation Theory; (2011). 13

[14] V. Futorny, D. Gratcharov, E. Ramirez; Classification of irreducible Gelfand-Tsetlin modules of sl(3); arXiv:1812.07137 [math.RT]. 5, 51, 56, 57

[15] B. Hall; Lie Groups, Lie Algebras, and Representations: An Elementary Introduction; Springer, (2015). 15 
[16] R. Hartshorne; Algebraic Geometry; Springer, (1977). 7, 24, 25, 26

[17] J. E. Humphreys; Representations of Semisimple Lie Algebras in the BGG Category $\mathcal{O}$; American Mathematical Society, (2008). 7, 9, 10, 11, 12, 17, 18, 20, 37

[18] D. Jordan; On the ideals of a Lie algebra of derivations; J. London Math. Soc., 33 (1986), 33-39. 3

[19] D. Jordan; On the simplicity of Lie algebras of derivations of commutative algebras, J. Algebra, 228 (2000), 580-585. 3

[20] V. Kac; Simple irreducible graded Lie algebras of finite growth, Math USSR-Izvestija 2 (1968), 1271-1311. 2

[21] W. Killing; Die Zusammensetzung der stetigen endlichen Transformationsgruppen; Math. Ann., 33 (1889), 1-48. 2

[22] J. Lagrange; Réflexions sur la résolution algébrique des équations; (Euvres complètes; 3 (1791), 205-421. 1

[23] M. Lau; Representations of multiloop algebras; Pacific J. Math., 245(1) 2010, 167184. 5

[24] E. Neher and A. Savage; A survey of equivariant map algebras with open problems; Contemp. Math. 602, Amer. Math. Soc., Providence, RI, (2013), 165182. 3

[25] O. Mathieu; Classification of Harish-Chandra modules over the Virasoro algebra; Invent. Math. 107 (1992), 225-234. 3, 31, 48

[26] A. Molev; Yangians and Classical Lie Algebras; Amer Mathematical Society, 2007. 20

[27] R. Moody; Lie algebras associated with generalized Cartan matrices; Bull. Amer. Math. Soc., 73, (1967), 217-222. 2

[28] S. Eswara Rao; Irreducible representations of the Lie algebra of the diffeomorphisms of a d-dimensional torus; J. Algebra, 182 (1996), 401-421. 4, 32, 52

[29] A. N. Rudakov; Irreducible representations of infinite-dimensional Lie algebras of Cartan type; Math. USSR Izvestija, 8 (1974), 836-866. 4, 43

[30] J. P. Serre; Linear Representations of Finite Groups; Springer (1977). 15 


\section{Index}

$\mathcal{A} \mathcal{V}$-module, 29

Affine algebraic variety, 22

Affine coordinate ring, 23

Cartan subalgebra, 9

Casimirs, 20

Center, 9

Central character, 20

Co-roots, 13

de Rham complex, 32

Derivation, 8

Dimension, 24

Dual representation, 18

Exterior algebra, 16

Fundamental weight, 20

Gauge fields, 32

Gauge module, 33

GT-module, 56

GT-subalgebra, 55

Harish-Chandra's theorem, 21

Hilbert's Nullstellensatz, 23

Irreducible representation, 14

Jacobian matrix, 25

Lie algebra, 7

Lie associative morphism, 8

Module, 14

PBW theorem, 17

Powers of the natural module, 18

Representation of a Lie algebra, 17

Representation of an associative algebra, 14

Root, 10

Root space, 10
Root system, 10

Smooth variety, 25

Tensor modules, 32

Triangular decomposition, 13 